GSI-Preprint-99-18, June 1999 (revised version)

\title{
Effective chiral theory of nucleon-nucleon scattering
}

\author{
Matthias Lutz ${ }^{a}$ \\ ${ }^{\mathrm{a}}$ GSI, 64220 Darmstadt, Germany \\ E-mail: m.lutz@gsi.de
}

\begin{abstract}
We present a new chiral expansion scheme for the nucleon-nucleon scattering amplitude which preserves unitarity exactly. Our effective field theory builds on the power counting rules for 2-nucleon reducible diagrams proposed in [1]. We evaluate the leading order terms of the isospin one scattering amplitude and elaborate in detail on the ${ }^{1} S_{0}$ phase shift. Our chiral description of the ${ }^{1} S_{0}$-phase shift does compete in quality with modern phenomenological nucleon-nucleon potentials. We describe elastic and inelastic scattering quantitatively up to laboratory energies of $E_{\text {lab }} \simeq 600 \mathrm{MeV}$.
\end{abstract}

\section{Introduction}

The nucleon-nucleon scattering problem has a long history in nuclear physics [2]. Nowadays there exist phenomenological nucleon-nucleon potentials [3-6] which successfully describe scattering data to high precision. These potentials are commonly constructed applying meson-exchange phenomenology. A serious drawback of the traditional approach is, however, the lack of a systematic scheme in the sense that there does not exist a well defined procedure to improve the potential. One has not yet identified a small expansion parameter which guides the construction of the potential nor has one resolved the renormalization problem. Results depend explicitly on ad-hoc formfactors. This will be an interesting issue when the data base on nucleon-nucleon scattering is further improved in the near future, in particular with upcoming experiments at the proton cooler facility COSY in Jülich.

A first attempt to systematically solve the problem of nucleon-nucleon scattering and construct a bridge to QCD was made by Weinberg [7]. He suggested to derive a nucleon-nucleon potential in time-ordered chiral perturbation theory [7]. The heavy meson-exchange contributions are, in accordance 
with chiral symmetry, systematically absorbed in local two-nucleon interaction vertices. This program was first carried out by Ordonez, Ray and van Kolck $[8,9]$ who computed nucleon phase shifts from the solution of a cutoff regularized Schrödinger equation feeded properly with the chiral potential. More recent attempts within the potential approach can be found in [10-12]. The chiral potential scheme is, however, still plagued with serious shortcomings. The systematic renormalization of the chiral potential scheme remains an open problem. This leads again to a regularization scheme dependence of physical results. Though in certain cases the regularization scheme dependence is found to be weak the reason for which awaits a systematic explanation.

In [1] the author suggested to apply a relativistic version of chiral perturbation theory $(\chi \mathrm{PT})$ with power counting rules also for two nucleon reducible diagrams. The proposed scheme is practicable since the 'difficult' non-local pion dynamics is found to be perturbative. Non perturbative effects are generated by properly renormalized local two-nucleon vertices. As a result the s-wave scattering amplitude is the sum of a pole term ( representing the deuteron bound state in the spin triplet channel or the pseudo-bound state in the spin singlet channel ) and a smooth remainder which is evaluated perturbatively in the pion dynamics. The question whether pions are perturbative in the nucleon scattering problem has been addressed also in $[13,14]$. Our scheme in [1] shows similarities with a framework proposed recently by Kaplan, Savage and Wise [15] and questioned by Cohen and Hansen [16]. Starting with the static one-pion exchange potential the authors [15] arrive at the power counting rules for reducible diagrams suggested in [1]. However, as will be shown in detail the implementation of the generalized chiral counting rules of [1] in [15] is in contradiction to the low energy structure of the nucleon-nucleon scattering amplitude. In particular unitarity is strongly violated. The s-wave scattering amplitude acquires a more complicated structure. Its representation in terms of a pole term and a smooth remainder accessed in terms of perturbative pions fails at unexpectedly small momenta. We will demonstrate that the failure of this scheme [1] in the spin singlet channel is caused by a second nonperturbative phenomenon present in the subthreshold scattering amplitude. The purpose of this work is to develop a consistent effective field theoretic application of the chiral power counting rules proposed in [1] in accordance with the low energy structure of the scattering amplitude.

We choose the relativistic version of the chiral Lagrangian since it offers on the one hand a manifest covariant framework and on the other hand it is the natural scheme to describe particle production processes like $p p \rightarrow p p \pi^{0}, p n \pi^{+}$. A further important novel ingredient of our scheme is a reorganization of the local two-body interaction vertices. Rather than expanding the interaction terms around zero three-momenta we propose to expand around a finite value. The physical motivation for this reorganization follows from the empirical observation that for example the ${ }^{1} S_{0}$ partial wave shows a zero at intermediate 
energies $E_{l a b .} \simeq 280 \mathrm{MeV}$. A novel systematic approximation scheme for the solution of the Bethe-Salpeter equation consistent with the chiral counting rules and unitarity is presented.

Our work is subdivided into four parts. In section two we discuss the chiral counting rules as they emerge from the relativistic chiral Lagrangian and introduce our expansion scheme for the nucleon-nucleon scattering amplitude. In section three we present analytic results of explicit evaluations at subleading orders. By evaluating the old scheme of [1] at three-loop level it is demonstrated that indeed it fails at momenta $p>m_{\pi}$. Many technical details of this calculation can be found in a series of appendices. In section four we extend our scheme to describe the pion production process and confront our results with the empirical scattering phase shift. We obtain a quantitative description

of elastic and inelastic s-wave nucleon-nucleon scattering in the spin singlet channel up to $E_{l a b} \simeq 600 \mathrm{MeV}$.

\section{Discussion of relativistic chiral power counting rules}

The chiral Lagrangian is constructed conveniently in terms of Weinberg's stereographic coordinates [21]. The buildings blocks are the pionic four vector $D_{\mu}$ with

$$
D_{\mu}^{c}=\frac{\partial_{\mu} \pi^{c}}{1+\lambda^{2} \pi^{2}}
$$

and the relativistic nucleon field $N$. The coupling $\lambda$ is identified with the chiral limit value of the pion decay constant $\lambda^{-1}=2 f_{\pi} \simeq 186 \mathrm{MeV}$. The chiral Lagrangian is now the infinite sum of all isoscalar terms formed with any number of the pion field $D_{\mu}$, nucleon fields $N$ and their respective covariant derivatives with:

$$
\begin{aligned}
\mathcal{D}_{\mu} N & =\left(\partial_{\mu}+i \frac{\lambda^{2}}{1+\lambda^{2} \pi^{2}} \vec{\tau} \cdot\left(\vec{\pi} \times \partial_{\mu} \vec{\pi}\right)\right) N \\
\mathcal{D}_{\mu} D_{\nu}^{a} & =\partial_{\mu} D_{\nu}^{a}+2 \lambda \pi^{a}\left(\vec{D}_{\nu} \cdot \vec{D}_{\mu}\right)-2 \lambda\left(\vec{\pi} \cdot \vec{D}_{\nu}\right) D_{\mu}^{a} .
\end{aligned}
$$

Further terms are to be added in order to describe the explicit chiral symmetry breaking pattern due to small current quark masses. Such terms do not add further complications to our discussion and therefore are not considered explicitly here. Weinberg's chiral power counting rules $[7,21]$ induce a systematic truncation of this infinite hierarchy. At a given order only a finite number of Feynman diagrams derived from a finite number of interaction terms need 
to be considered. Since we will generalize Weinberg's counting rules we briefly recall his scheme.

For a given irreducible Feynman diagram one introduces the number of internal nucleon lines, $I_{N}$, the number of internal pion lines, $I_{\pi}$, the number of loops, $L$ and the number, $V_{i}$, of vertices of type $i$ with $d_{i}$ 'small' derivatives involved. Here one calls a derivative small if it acts on the pion field. The chiral counting rule for a given Feynman diagram results if the nucleon propagator is given the chiral power -1 , the pion propagator -2 , a small derivative the power 1 and the loop momentum the power 4 . The usefulness of the counting rules (3) follows by means of the topological relations $L=I_{\pi}+I_{N}-\sum_{i} V_{i}+1$ and $2 I_{N}+E_{N}=\sum V_{i} n_{i}$ where the number of external nucleon lines $E_{N}$ and $n_{i}$, the number of nucleon fields in an interaction term of type $i$ is introduced. The well know result

$$
\begin{aligned}
\nu & =4 L-I_{N}-2 I_{\pi}+\sum_{i} V_{i} d_{i} \\
& =2-\frac{1}{2} E_{N}+2 L+\sum_{i} V_{i}\left(d_{i}+\frac{1}{2} n_{i}-2\right)
\end{aligned}
$$

shows that for $d_{i}+\frac{1}{2} n_{i}-2 \geq 0$, higher loop diagrams are more and more suppressed since they carry larger and larger chiral powers. We emphasize that the counting rule (3) is not applicable for multi-nucleon reducible diagrams which one encounters for example in the iteration of the Bethe-Salpeter equation for the nucleon-nucleon scattering amplitude.

There are two problems inherent with the relativistic approach. First, any covariant derivative acting on the nucleon field produces the large nucleon mass and therefore must be assigned the minimal chiral power zero. Thus an infinite tower of interaction terms needs to be evaluated at given finite chiral order $[17,18]$. Second, the straightforward evaluation of Feynman diagrams involving relativistic nucleon propagators generates positive powers of the large nucleon mass [17]. Therefore the chiral power counting rule (3) is spoiled. The relativistic scheme appeared impractical and the heavy mass formulation of $\chi \mathrm{PT}$, which overcomes both problems by performing a non relativistic $1 / \mathrm{m}$ expansion at the level of the Lagrangian density, was developed [19] and applied extensively in the one nucleon sector [20]. In the two-nucleon sector, however, the heavy baryon mass formulation of $\chi \mathrm{PT}$ is not applicable due to the ill-defined two-nucleon reducible diagrams [7]. Weinberg circumvented those problems by deriving chiral power counting rules for time-ordered perturbation theory [7].

In this work we follow a different path and work directly with the relativistic form of the chiral Lagrangian [1]. This has the advantage that it is possible to control covariance at each step of the calculation. We outline how the 
two aforementioned pertinent problems can be overcome within the framework of the relativistic chiral Lagrangian. We expect our relativistic scheme to be equivalent to the more familiar heavy fermion formulation of chiral perturbation theory in the one-nucleon sector. Rather than performing the $1 / \mathrm{m}$ expansion at the level of the Lagrangian density we suggest to work out this expansion explicitly at the level of individual relativistic Feynman diagrams. Therewith we avoid the heavy baryon chiral Lagrangian with its known artifacts in the two-nucleon sector.

It is convenient to consider the chiral Lagrangian (at an intermediate stage) to represent a finite cutoff theory [7]. The finite cutoff, $\Lambda \ll m$, is required to restrict the nucleon virtuality inside a given loop diagram such as to justify the $1 / m$ expansion. Obviously a finite cutoff has to be introduced with great care not to break any chiral Ward identity. Alternatively one may apply dimensional regularization $[37,38]$. We emphasize that if dimensional regularization is applied one has to first expand in $1 / m$ and then perform the loop integration. The two steps do not commute here. This is equivalent to first performing the $1 / m$ expansion and then applying the large cutoff limit in a renormalized finite cutoff theory. This procedure suppresses contributions from loop momenta larger than the nucleon mass which are the source for both the conflict of relativistic diagrams with the counting rule (3) and the appearance of multiple powers of the nucleon mass [17].

It remains to demonstrate that the relativistic chiral Lagrangian leads to a practical scheme in the sense that at a given chiral order $Q$ there are only a finite number of diagrams to be considered. Here it is convenient to generalize the notation of (3) and denote with $d_{i}$ the chiral order of an interaction vertex of type $i$. We will show that by an appropriate regrouping of interaction terms in the chiral Lagrangian there are only a finite number of renormalized interaction vertices of given chiral order. Consider a covariant derivative $\mathcal{D}_{\mu}(x)$ acting on a nucleon field $N(x)$. We need to construct a suitable 'counter interaction' leading to a 'small' renormalized vertex. This crucial rearrangement is illustrated at hand of the simplest case when the Lorentz index $\mu$ is saturated by another covariant derivative acting on the same nucleon field. In this case the counter interaction is readily found

$$
\mathcal{D}^{\mu}(x) \mathcal{D}_{\mu}(x) N(x) \rightarrow\left(\mathcal{D}^{\mu}(x) \mathcal{D}_{\mu}(x)+m^{2}\right) N(x) \sim Q
$$

The 'rearranged' vertex is proportional to the virtuality of the nucleon, $\left(\partial_{\mu} \partial^{\mu}+\right.$ $\left.m^{2}\right) N$, which is of 'minimal' chiral order $Q$. It is important to observe that this counter interaction is part of the chiral Lagrangian by construction. In the more general case where there are $2 k$ derivatives acting on a nucleon field $\left(\mathcal{D}^{\mu}(x) \mathcal{D}_{\mu}(x)\right)^{k} N(x)$ an appropriate rearrangement leads to a renormalized structure $\left(\mathcal{D}^{\mu}(x) \mathcal{D}_{\mu}(x)+m^{2}\right)^{k} N(x)$. The total suppression factor of the in- 
teraction vertex $d_{i}=d_{i}^{(\pi)}+d_{i}^{(N)}$ is the sum of the number of derivatives acting on pion fields $d_{i}^{(\pi)}$, and the degree of virtuality of the nucleon field $d_{i}^{(N)}=k$. Note that a similar construction is applicable if covariant derivatives acting on different nucleon fields meet.

We are left to consider the case in which the Lorentz index $\mu$ of the covariant derivative $\mathcal{D}_{\mu}$ is contracted either with the index of a Dirac matrix or the index of a derivative acting on a pion field. In the latter case vertices are well behaved since they are already suppressed by the pion derivative in $\partial_{\mu} \pi$. Of course, here, one has to give the nucleon derivative the chiral power $Q^{0}$. The 'large' nucleon mass is not 'hazardous' if one identifies the natural scale of any coupling constant $\bar{\Lambda}$ to be of the same order as the nucleon mass. With $\bar{\Lambda} \simeq m_{\rho}$ this seems indeed reasonable. The remaining case in which the nucleon derivative couples to the index of a Dirac matrix in a vertex does not generate an infinite tower of interaction terms since at a given number of nucleon fields $n_{i}$, the number of available indices provided by Dirac matrices is at most $n_{i}$. The degree of nucleon virtuality can be determined upon inspection case by case.

The above arguments show how to organize the infinite tower of chiral interaction terms such that there is always only a finite number of interaction terms of a given chiral power. The counting rule (3) applies where $d_{i}$ is identified with the sum of number of derivatives acting on pion fields and the degree of nucleon virtuality of the vertex as discussed above. Thus at any given chiral order only a finite number of diagrams need to be evaluated. We note that in the relativistic scheme it is convenient to use the notion of 'minimal' chiral power of a Feynman diagram since a given diagram leads to an infinite tower of terms with increasing chiral powers upon expansion. The minimal chiral power is given by the counting rule (3).

\subsection{2-Particle reducible diagrams}

In this section we discuss the 2-particle reducible diagrams for which 'standard' chiral power counting rules are not applicable [1,7]. In our relativistic framework one is therefore bound to consider the Bethe-Salpeter equation for the nucleon-nucleon scattering. This is analogous to Weinberg's approach [7] which considers the Lippmann-Schwinger equation with a suitable potential expanded according to chiral power counting rules.

The nucleon-nucleon scattering amplitude $T$, is given in terms of the BetheSalpeter kernel $K$ 


$$
S=2 C+K=\frac{2}{1-K G} K
$$

where for convenience we use operator notation in the last expression. The scattering amplitude $T$ and interaction kernel $K$ of (5) are antisymmetric under exchange of the ingoing or outging nucleons. The factor '2' in (5) leads to the proper treatment of exchange diagrams. The interaction kernel

$$
K=K+\underset{K}{K}+\ldots
$$

is the sum of two-particle irreducible diagrams deduced from the relativistic chiral Lagrangian. To leading zeroth order it is the sum of local interaction terms and the one-pion exchange diagram displayed in (6) with a wavy line representing the relativistic pion propagator. The object $G$ in (5) represents the relativistic two-nucleon propagator. Let us investigate the Bethe-Salpeter equation (5) perturbatively. In the following we introduce the appropriate minimal chiral power $\nu$ for $K G K$ with respect to a selected part of the interaction kernel $K$ carrying the minimal chiral power $\nu_{K}$. The once iterated Bethe-Salpeter kernel is

$$
K G K \sim \int \frac{d^{4} l}{(2 \pi)^{4}} \frac{2 m K(l)}{\left(\frac{1}{2} w-l\right)^{2}-m^{2}+i \epsilon} \frac{2 m K(l)}{\left(\frac{1}{2} w+l\right)^{2}-m^{2}+i \epsilon}
$$

with $w^{2}=s$. In the center of mass frame with $\vec{w}=0$ the Mandelstam variable $s=4 m^{2}+4 p^{2}$ is given by the relative momentum $p$ of the nucleons. It is instructive to study first the s-channel spectral density, $\rho(s)$, of this contribution. It typically receives strength from the pinch singularity generated by the two-nucleon propagators in (7). In the center of mass frame one finds:

$$
\rho(s)=\left.\Im(K G K) \sim \frac{p}{\sqrt{m^{2}+p^{2}}} \frac{m^{2}}{4 \pi} \int \frac{d \Omega_{\vec{l}}}{4 \pi} K(\vec{l}) K(\vec{l})\right|_{l_{0}=0,|\vec{l}|=p} .
$$

Expression (8) can now be used to introduce the minimal chiral power $\nu$ of the s-channel spectral density

$$
\nu=1+2 \nu_{K}
$$

in terms of the minimal chiral power $\nu_{K}$ of the Bethe-Salpeter kernel $K$ given by (3). In (9) we exploit that the elastic nucleon-nucleon phase space leads to the small momentum $p$ in the spectral density. Note that the angle average in (8) does not affect the chiral power. If one assigns $K \sim 1 /\left(4 f_{\pi}^{2}\right)$, as suggested by the one-pion exchange contribution to the kernel, and identifies $m \simeq 4 \pi f_{\pi}$ 
the dimensionless expansion parameter $p /\left(4 f_{\pi}\right)$ arises. This indicates convergence for small momenta $p<350 \mathrm{MeV}$. Note, however, that if the kernel $K(p)$ in (8) vanishes for large momenta $p$ we expect convergence possibly also for $p>350 \mathrm{MeV}$. This is typically the case if the spectral function $\rho(s)$ leads to a finite loop $\Re K G K$ (see $(11,41)$ ). We then expect the dimensionless parameter $m_{\pi} /\left(4 f_{\pi}\right) \simeq 0.4$ since the falloff in $K(l)$ must be driven by the pion mass. It is instructive to compare the chiral power (9) with the 'naive' chiral power, $2+2 \nu_{K}$, implied by the standard counting rule (3). This confirms that the counting rule (3) must not be applied to all parts of two-nucleon reducible diagrams. We point out that inelastic contributions to $\rho(s)$, for example from the one-pion production cut, can be treated in full analogy to the two-nucleon cut. The chiral power of the contribution to the spectral density follows upon inspection of the production phase space. In fact here the counting rule (3) leads to the correct minimal chiral power (see appendix B.4 and B.5 for explicit examples).

To have a useful scheme we also need to derive counting rules for the real part of the scattering amplitude. Here we invoke causality which relates the real part of a given Feynman diagram to its imaginary part by means of a dispersion relation ${ }^{1}$

$$
I(s)=\Re(K G K)=\frac{1}{\pi} \mathcal{P} \int_{4 m^{2}}^{\infty} d \bar{s} \frac{\rho(\bar{s})}{\bar{s}-s} .
$$

The dispersion relation (10) need not to be finite and well defined. It may be ultraviolet divergent. However, one is free to consider the chiral Lagrangian as a finite cutoff theory $[7,22]$. If the cutoff $\Lambda$ is to be interpreted as a bound for the maximal virtuality of nucleons one should first remove the nucleon rest mass. Therefore we take the cutoff $\Lambda$ to restrict the momentum $p \leq \Lambda$ rather than the Mandelstam variable $s$ in the dispersion relation (10):

$$
I(p, \Lambda)=\frac{1}{\pi} \mathcal{P} \int_{4 m^{2}}^{4\left(m^{2}+\Lambda^{2}\right)} d \bar{s} \frac{\rho(\bar{s})}{\bar{s}-s}=\frac{1}{\pi} \mathcal{P} \int_{0}^{\Lambda^{2}} d \bar{p}^{2} \frac{\rho\left(4 m^{2}+4 \bar{p}^{2}\right)}{\bar{p}^{2}-p^{2}}
$$

1 The iterated Bethe-Salpeter kernel does not necessarily satisfy a s-channel dispersion relation. However, we point out that only the part which provides strength for the s-channel spectral density requires special attention and modified power counting rules. Obviously a part which does not generate strength for the s-channel spectral density has no pinch singularity leading to a s-channel cut so that standard counting rules apply. Note that for a given contribution one has to specify whether the dispersion relation holds at fixed Mandelstam variable $t$ or $u$. This technical detail, however, does not affect our argument. 
Formally one counts $m_{\pi} / \Lambda \sim \Lambda / m \sim Q$ with $m_{\pi} \ll \Lambda<m$ [7]. The natural guess for the chiral power of the integral $I(p, \Lambda)$ would be the chiral power of its imaginary part. However, the integral (11) as it stands does not lead to this chiral power yet. Though the separation of scales is manifest in the spectral density $\rho(s)$ the cutoff scale $\Lambda$ prevents the conclusion that the integral behaves like a small scale to the power $1+2 \nu_{K}$. We point out that after an appropriate number of subtractions at $p^{2}=-\mu^{2}<0$ with $\mu \sim m_{\pi}$ the counting rule (9) can nevertheless be applied to the real part of the loop function. The subtraction is required to render the dispersion integral finite in the large cutoff limit:

$$
I_{S}^{(n)}(p, \Lambda ; \mu)=\frac{1}{\pi} \mathcal{P} \int_{0}^{\Lambda^{2}} d \bar{p}^{2} \frac{\rho\left(4 m^{2}+4 \bar{p}^{2}\right)}{\bar{p}^{2}-p^{2}}\left(\frac{p^{2}+\mu^{2}}{\bar{p}^{2}+\mu^{2}}\right)^{n}
$$

The subtraction polynomial can always be absorbed into the contact 4-nucleon vertices. Note that the performed subtractions do not necessarily simplify our attempt to derive a counting rule since this procedure introduces a new scale, $\mu$, the subtraction point. Nevertheless we now can exploit the freedom to choose the subtraction point. If we insist on a 'small' subtraction scale $\mu \sim m_{\pi}$ of the order of the pion mass one can apply dimensional power counting since the integral is finite in the large cutoff limit by construction.

The above arguments establish the chiral power of a once iterated BetheSalpeter kernel. The general case of a n-times iterated kernel follows by complete induction provided that a suitable subtraction mechanism (see section 2.4 ) is implemented into the Bethe-Salpeter equation. We arrive at the desired chiral counting rule for 2-particle reducible diagrams: each pair of nucleon propagators which exhibit an s-channel unitarity cut receives the chiral power -3 in contrast to the 'naive' power -2 . Therefore the n-times iterated Bethe-Salpeter kernel receives the minimal chiral power

$$
\nu_{K}^{(n)}=n+n \nu_{K}
$$

We stress, first, that this counting rule is manifest only if one introduces a 'small' subtraction scale $\mu \sim m_{\pi}$ at the level of the Bethe-Salpeter equation and second, that the subtraction scale is independent of the intrinsic cutoff. We will refer to this counting rule as the 'L'-counting rule since for the onepion exchange interaction, which contributes at order zero to the interaction kernel, the chiral power of the n-th iterated interaction is simply given by the number of loops. In appendix B the reader may find explicit examples which confirm our L-counting rule (13).

We summarize what we derived. The non-polynomial part of the once iterated Bethe-Salpeter kernel has an excess of one chiral power as compared to the sum 
of the chiral powers of the iterated Bethe-Salpeter kernels (see (9)). We point out that this result, though obtained by means of dispersion relations, holds also if a different technique for the evaluation of the loop functions is applied. Since the chiral power of the Bethe-Salpeter kernel starts at $\nu=0$ our counting rules seem to suggest that the Bethe-Salpeter kernel has to be iterated only a finite number of times at a given chiral order $\nu$ for the scattering amplitude. Obviously this can not be correct since there are well known non-perturbative effects present in the scattering amplitude like for example the deuteron bound state. This puzzle can be resolved by realizing that the implicit assumption of any chiral power counting scheme is the naturalness of all coupling constants carrying non trivial dimensions. The characteristic scale is assumed to be the mass of the lightest degree of freedom not explicitly included in the effective chiral Lagrangian.

Therefore the crucial question to ask is: does the suggested subtraction scheme for the Bethe-Salpeter equation affect the naturalness of the 4-nucleon coupling strengths?

\subsection{Mutation of natural scale to anomalous scale}

In this section we address the question to what extent a subtraction scheme affects the naturalness of the bare coupling function. The effect of our subtraction scheme is illustrated at hand of s-wave scattering in the isospin one channel. We consider here a theory without explicit pions defined by an infinite tower of separable interactions in the ${ }^{1} S_{0}$-channel ${ }^{2}$ :

$$
\mathcal{L}_{4 N}(x)=\frac{1}{4} \sum_{n=0}^{\infty} g^{(2 n)} \partial^{n}\left(\bar{N} \gamma_{5} C \tau \tau_{2} \bar{N}^{t}\right) \partial^{n}\left(N^{t} \tau_{2} \tau C^{-1} \gamma_{5} N\right)
$$

with the charge conjugation matrix $C=i \gamma_{0} \gamma_{2}$ and the isospin Pauli matrices $\tau_{i}$. The index contractions of the derivatives $\partial_{\mu}$ in (14) are such that in momentum space the energy dependent coupling $g(s)$ arises:

$$
g(s)=\sum_{n=0}^{\infty} g^{(2 n)} s^{n}=\sum_{n=0}^{\infty} g_{2 n}\left(s-4 m^{2}\right)^{n}
$$

2 Note that the terms displayed in (14) are the only ones relevant for s-wave scattering in the spin singlet channel. Any further terms necessarily are on-shell equivalent to terms already included and lead to relativistic nucleonic tadpole diagrams upon unitary iteration in the Bethe-Salpeter equation. Such terms can be dropped in a consistent treatment (see appendix B.1 and (46)). Note that the situation is different in a non-relativistic framework where one encounters a richer variety of interaction terms $[26]$. 
with $p^{2}=s / 4-m^{2}$. In (15) we introduce renormalized couplings $g_{2 n}$ according to the mandatory regrouping of interaction terms discussed in section 2 . Note that in the derivation of Feynman rules from (14) the troublesome timederivatives can be treated naively since the form of the final effective Feynman vertex is dictated by covariance. At tree level the coupling $g_{0}$ can be interpreted in terms of the s-wave scattering lengths $a\left({ }^{1} S_{0}\right)$ with $2 m g_{0}=-4 \pi a\left({ }^{1} S_{0}\right)$. Recall that one may apply a phenomenological saturation model for the coupling function with $g(s)$ receiving contributions from heavy meson $(\sigma, \rho, \omega, \ldots)$ exchanges. Such a model provides a bridge to the traditional meson-exchange picture of nucleon-nucleon scattering. It furthermore motivates the crucial assumption of any chiral expansion, namely, that all couplings are natural: in particular we expect $g_{n+2} \sim g_{n} / \Lambda_{\text {nat. }}^{2}$. We identify here the natural scale $\Lambda_{\text {nat }}$. with one half of the mass of the lightest meson not included explicitly in the theory. The factor $1 / 2$ follows since the t-channel meson-exchange diagram in a given partial wave suggests a convergence radius of half the meson mass for an expansion around $p^{2}=0$. In a theory with pions one identifies $\Lambda_{\text {nat. }}=m_{\rho} / 2$ and in a theory where pions are integrated out $\Lambda_{\text {nat. }}=m_{\pi} / 2$. It is worth emphasizing that the leading term requires special attention since $g_{0} \neq 1 / \Lambda_{\text {nat. }}^{2}$. For example the vector meson $u$ and $t$ channel exchange diagrams predict $g_{0} \sim-g_{\rho N N}^{2} /\left(8 m_{\rho}^{2}\right)-g_{\omega N N}^{2} /\left(8 m_{\omega}^{2}\right) \sim-1 /\left(8 f_{\pi}^{2}\right)$ with a typical meson-nucleon coupling constant $g_{\omega N N} \sim g_{\rho N N} \sim m_{\rho} /\left(\sqrt{2} f_{\pi}\right)$ estimated with the KSFR relation. Therefore one expects $g_{0}=\lambda^{2} \hat{g}_{0}$ with the dimensionless parameter $\hat{g}_{0}$ of order unity.

We proceed and solve the Bethe-Salpeter equation for the nucleon-nucleon scattering amplitude $T$, with the tree-level Bethe-Salpeter kernel of (14). The equation is separable and therefore the solution can be written in terms of the divergent one-loop integral, $J(s, \Lambda)$,

$$
\begin{aligned}
T(p) & =\frac{g(s)}{1-g(s) J(s)}\left(\tau \tau_{2} \gamma_{5} C^{-1}\right) \times\left(C \gamma_{5} \tau_{2} \tau\right) \\
J(s, \Lambda) & =-i \operatorname{tr} \int \frac{d^{4} l}{(2 \pi)^{4}} C^{-1} \gamma_{5} S_{F}\left(l+\frac{1}{2} w\right) \gamma_{5} C S_{F}^{t}\left(-l+\frac{1}{2} w\right)
\end{aligned}
$$

Here $S_{F}(p)=1 /(\not p-m+i \epsilon)$ is the relativistic nucleon propagator and $w^{2}=4\left(m^{2}+p^{2}\right)$. The loop integral may be evaluated by writing a dispersion integral ${ }^{3}$

$\overline{3}$ We are aware that the introduction of a finite cutoff in Feynman diagrams requires special care in order not to break the pertinent symmetries. For more details we refer to the appendix. 


$$
J(s, \Lambda)=\frac{1}{\pi} \int_{0}^{\Lambda^{2}} \frac{d \bar{p}^{2} \rho_{J}(\bar{p})}{\bar{p}^{2}-p^{2}-i \epsilon}
$$

where we restrict the relative momentum of the center of mass system by the finite cutoff parameter $\Lambda$. The well defined spectral density is:

$$
\rho_{J}(p)=\frac{m p}{2 \pi} \sqrt{1+\frac{p^{2}}{m^{2}}}=\frac{m p}{2 \pi}\left(1+\frac{p^{2}}{2 m^{2}}+\mathcal{O}\left(\frac{p^{4}}{m^{4}}\right)\right) .
$$

We expand the loop integral $J(s, \Lambda)$ non relativistically in powers of $1 / m$ by expanding its imaginary part (18). Here we encounter a simple example how to systematically expand a Feynman diagram given by the relativistic chiral Lagrangian. Consider for example the loop function at zero momentum

$$
J\left(4 m^{2}, \Lambda\right)=\frac{1}{2 \pi^{2}}\left(\Lambda \sqrt{m^{2}+\Lambda^{2}}+m^{2} \operatorname{arcsinh}\left(\frac{\Lambda}{m}\right)\right)
$$

A naive evaluation of the diagram with $\Lambda \gg m$ would generate a troublesome multiple power of the nucleon mass with $J \sim m^{2} \log \Lambda$ counteracting any chiral counting rule. Equations (17) and (19) exemplify the necessity of the intrinsic cutoff $\Lambda$ to be smaller than the nucleon mass such that the $1 / m$ expansion is justified. Expanding (19) in $\Lambda / m$ reduces the leading power of $m$. This is also seen if the real part of the loop $J(s, \Lambda)$ is evaluated with the expanded spectral function (18) by means of the dispersion integral (17) :

$$
\begin{aligned}
\Re J(s, \Lambda) & =\frac{m}{\pi^{2}} \Lambda\left(1+\frac{\Lambda^{2}}{6 m^{2}}+\mathcal{O}\left(\frac{\Lambda^{4}}{m^{4}}\right)\right) \\
& +\frac{m}{\pi^{2}} \frac{p^{2}}{\Lambda}\left(1+\frac{\Lambda^{2}}{2 m^{2}}-\frac{\Lambda^{4}}{24 m^{4}}+\mathcal{O}\left(\frac{\Lambda^{6}}{m^{6}}\right)\right)+\mathcal{O}\left(p^{4}\right) .
\end{aligned}
$$

It is worth pointing out that the subleading terms in the $1 / m$ expansion proportional to $(\Lambda / m)^{n}$ with $n>1$ can be evaluated with $\Lambda=m$. This follows since the $\Lambda / m$ expansion is controlled typically by square root singularities $\sqrt{m^{2}+p^{2}}$ for which even at $\Lambda / m=1$ the non-relativistic expansion (18) is converging rapidly. This is nicely confirmed by the suppression factors $1 / 6$ and $1 / 12$ for the constant term and $p^{2}$-term in (20) respectively. Therefore, effectively we are left with only linear divergencies in $\Lambda$. In fact it is legitimate to drop all terms $(\Lambda / m)^{n}$ with $n>1$ since at given order $p^{2 k}$ their effect can be compensated by a small change of $\Lambda$. This is particularly convenient since then nucleonic tadpole diagrams can be dropped systematically. With this simplified regularization scheme we avoid the problems one encounters in cutoff-regularized effective field theory. The ill-defined multiple powers of the cutoff are eliminated and need not to be canceled by the careful construction 
of the cutoff dependence of path integral measure [27]. For more details on our regularization procedure including or more rigorous definition we refer the reader to appendix $B$. Here we point at a further important observation to be made. The presence of terms like $(p / \Lambda)^{2 k}$ necessarily leads to the identification $\Lambda \simeq \Lambda_{\text {nat. }}$. Only then such terms can be absorbed into contact interaction terms of the chiral Lagrangian.

To leading order in the non-relativistic expansion the one-loop integral (17) can be rendered finite by one subtraction at $s=4 m^{2}-\mu^{2}$

$$
J_{S}(s, \Lambda ; \mu)=J(s, \Lambda)-J\left(4 m^{2}-\mu^{2}, \Lambda\right)=\frac{1}{\pi} \int_{0}^{\Lambda^{2}} \frac{d \bar{p}^{2} \rho_{J}(\bar{p})}{\bar{p}^{2}-p^{2}-i \epsilon} \frac{p^{2}+\mu^{2}}{\bar{p}^{2}+\mu^{2}}
$$

where the subtraction constant $J\left(4 m^{2}-\mu^{2}, \Lambda\right)$ can be absorbed into the coupling function $g(s)=g(s, \Lambda)$. Note that subleading terms in the $1 / m$ expansion require further subtractions. The subtracted loop (21) is not identified with the renormalized loop. It is more convenient to deal with a manifest cutoff independent renormalized loop function. We introduce $J_{R}(p, \mu)=J_{S}(s, \infty ; \mu)$ and accordingly the renormalized coupling $g_{R}\left(p^{2}, \mu\right)$ with

$$
T_{\left[{ }^{1} S_{0}\right]}(p)=\frac{1}{g^{-1}(s, \Lambda)-J(s, \Lambda)}=\frac{1}{g_{R}^{-1}\left(p^{2}, \mu\right)-J_{R}(p, \mu)} .
$$

All dependence on the way in which the cutoff $\Lambda$ was introduced is absorbed into the bare coupling function $g(s, \Lambda)$. Consequently the choice of the cutoff scheme does not have any effect as long as the scheme does not break the pertinent symmetries. The leading order renormalized loop reads

$$
J_{R}(p, \mu)=\frac{m}{2 \pi^{2}} \int_{0}^{\infty} \frac{d \bar{p}^{2} \bar{p}}{\bar{p}^{2}-p^{2}-i \epsilon} \frac{p^{2}+\mu^{2}}{\bar{p}^{2}+\mu^{2}}=\frac{m}{2 \pi}(\mu+i p)
$$

where we dropped all $1 / m$ correction terms in $(23)$. The leading order renormalized coupling function is:

$$
\begin{aligned}
g_{R}^{-1}\left(p^{2}, \mu\right) & =g^{-1}(s, \Lambda)-\frac{m}{2 \pi^{2}} \int_{0}^{\Lambda^{2}} \frac{d \bar{p}^{2} \bar{p}}{\bar{p}^{2}+\mu^{2}}+\frac{m}{2 \pi^{2}} \int_{\Lambda^{2}}^{\infty} \frac{d \bar{p}^{2} \bar{p}}{\bar{p}^{2}-p^{2}} \frac{p^{2}+\mu^{2}}{\bar{p}^{2}+\mu^{2}} \\
& =g^{-1}(s, \Lambda)-\frac{m}{\pi^{2}}\left(\Lambda-\frac{\pi}{2} \mu\right)+\frac{m}{\pi^{2}} \frac{p^{2}}{\Lambda}\left(1+\mathcal{O}\left(p^{2} / \Lambda^{2}\right)\right) .
\end{aligned}
$$

It is important to observe that for $p \leq \Lambda$ the contribution form the loop function to the renormalized coupling function can be expanded in powers of the 
small momentum $p$. Our result (24) demonstrates that all cutoff dependence can safely be absorbed in the bare coupling $g(s, \Lambda)$ provided that it is legitimate to identify the typical cutoff $\Lambda$ with the natural scale of the bare coupling function $\Lambda_{\text {nat. }}$. Equation (24) exhibits an interesting effect. Starting with a natural bare coupling function $g(s, \Lambda)$ it potentially generates an anomalously large renormalized coupling $g_{R}\left(p^{2}=0, \mu\right)$, however, only if the subtraction scale $\mu$ is taken to be sufficiently small as required by the L-counting rule. Then an attractive natural coupling $g^{-1}\left(4 \mathrm{~m}^{2}, \Lambda\right)>0$ potentially cancels the $m \Lambda$ term in (24) predicting an anomalously large renormalized coupling $g_{R}(0, \mu) \sim 2 \pi /(m \mu)$. We stress that this is a familiar phenomenon since it is long understood that an attractive system may dynamically generate 'new' scales which are anomalously small (like for example the binding energy of shallow bound state). What is intriguing here is the transparent and simple mechanism provided by (24) to naturally generate such a 'small' scale $[1,28]$. If the cancellation in (24) is manifest for a physical system the renormalized coupling $g_{R}(0, \mu)$ must be assigned an anomalous chiral power, e.g. the 'minimal' chiral power -1 at given subtraction scale with $\mu \sim m_{\pi}$.

In $(24)$ we refrained from expanding the inverse bare coupling function $g^{-1}(s, \Lambda)$. We point out that it is necessary to discriminate two scenarios. In scenario I) the convergence radius of $g^{-1}(s, \Lambda)$ is set by the appropriate natural scale $\Lambda_{\text {nat }}^{(I)}$. leading to a well behaved Taylor series in $p^{2}$. In scenario II) the convergence radius of $g^{-1}(s, \Lambda)$ is set by a zero of the bare coupling function at $p^{2}=\mu_{\sigma}^{2}$ with $\mu_{\sigma}<\Lambda_{\text {nat. }}^{(I)}$. In this case it may be advantageous to expand the renormalized coupling around $p=\mu_{\sigma}$. If one insists on expanding around $p=0$ the theory breaks down at scale $p \leq \mu_{\sigma}<\Lambda_{\text {nat. }}^{(I)}$. In fact expanding around $p=\mu_{\sigma}$ further increases the convergence radius with $\Lambda_{\text {nat. }}^{(I I)}=\Lambda_{\text {nat. }}^{(I)}+\mu_{\sigma}$ simply because the distance of the expansion point $p=\mu_{\sigma}$ to the closest subthreshold singularity at $p= \pm i \Lambda_{\text {nat. }}^{(I)}$ is increased. Note that here we implicitly assume that $\mu_{\sigma}+\Lambda_{\text {nat }}^{(I I)}$. is smaller than the one-particle production cut with $2 \mu_{\sigma}^{2}+\mu_{\sigma} \Lambda_{\text {nat. }}^{(I)}<m \Lambda_{\text {nat. }}^{(I)}$. For example, in an effective theory which treats pions explicitly and where the $\rho$-meson is the lightest relevant degree-of-freedom that is integrated out, the optimal expansion point $p \simeq-m_{\rho} / 4+\left(m m_{\rho} / 4+m_{\rho}^{2} / 16\right)^{1 / 2} \simeq 275 \mathrm{MeV}$ leads to the maximal convergence radius or equivalently a natural scale of $\Lambda_{\text {nat. }} \simeq 660 \mathrm{MeV}$.

We investigate the scattering amplitude $T_{\left[{ }^{1} S_{0}\right]}(p)$ of $(22)$ in the two scenarios to leading order in the $1 / m$ expansion

$$
\begin{aligned}
& T_{\left[{ }^{1} S_{0}\right]}^{(I)}(p)=\left(g_{R, I}^{(0,-1)}(\mu)+g_{R, I}^{(2,-1)} p^{2}+\cdots-\frac{m}{2 \pi}(\mu+i p)\right)^{-1}, \\
& T_{\left[{ }^{1} S_{0}\right]}^{(I I)}(p)=\left(\frac{g_{R, I I}^{(-2,-1)}}{p^{2}-\mu_{\sigma}^{2}}+g_{R, I I}^{(0,-1)}(\mu)+g_{R, I I}^{(2,-1)}\left(p^{2}-\mu_{\sigma}^{2}\right)\right.
\end{aligned}
$$




$$
\left.+\cdots-\frac{m}{2 \pi}(\mu+i p)\right)^{-1}
$$

where we implicitly assume that the bare coupling shows a simple zero with $g\left(\mu_{\sigma}^{2}\right)=0$ but $g^{\prime}\left(\mu_{\sigma}^{2}\right) \neq 0$. In principal a zero with higher degeneracy can not be excluded though it is less likely for a natural coupling function. Multiple zeros of $g\left(p^{2}, \Lambda\right)$ are difficult to reconcile with the naturalness assumption. We emphasize that an effective field theory formulated with an explicit cutoff may give rise to a similar behavior since here the inverse bare coupling is kept and not expanded. The zero at $p^{2}=\mu_{\sigma}^{2}$, however, is then necessarily a consequence of strongly correlated and fine-tuned parameters resulting from a fit to data. In our scheme (25) we avoid a strong correlation of parameters since the vanishing of the phase shift is already build in and controlled directly by $\mu_{\sigma}$. There is a further important difference to our scheme: at order $Q^{2}$ the inverse scattering amplitude of scenario I) requires two parameters whereas scenario II) leads to four parameters. In other words scenario II) predicts the relevance of more operators at a given order.

It is instructive to explicitly work out the implications of the naturalness assumption and discuss the low energy structure of $T_{\left[{ }^{1} S_{0}\right]}(p)$ at momenta $p \ll$ $\mu_{\sigma}$ in both scenarios. It leads to coupling functions of the form:

$$
\begin{aligned}
g_{R, I}^{(0,-1)}(\mu) & =-\frac{m}{\pi^{2}}\left(\Lambda_{\text {nat. }}^{(I)}-\frac{\pi}{2} \mu\right)+\hat{g}_{R, I}^{(0,-1)} 4 f_{\pi}^{2}, \\
g_{R, I I}^{(-2,-1)} & =\hat{g}_{R, I I}^{(-2,-1)} 4 f_{\pi}^{2}\left(\Lambda_{\text {nat. }}^{(I I)}\right)^{2}, \\
g_{R, I I}^{(0,-1)}(\mu) & =-\frac{m}{\pi^{2}}\left(\Lambda_{\text {nat. }}^{(I I)}-\frac{\pi}{2} \mu\right)+\hat{g}_{R, I I}^{(0,-1)} 4 f_{\pi}^{2}
\end{aligned}
$$

where the dimensionless quantities $\hat{g}_{R, I I}^{(-2,-1)}, \hat{g}_{R, I I}^{(0,-1)}$ and $\hat{g}_{R, I}^{(0,-1)}$ are of order unity. We point out that $g_{R, I I}^{(0,-1)} \sim 4 f_{\pi}^{2}$ and $g_{R, I}^{(0,-1)}(\mu) \sim 4 f_{\pi}^{2}$ holds only for $\mu \simeq \Lambda_{\text {nat. }}^{(I)}$ and $\mu \simeq \Lambda_{\text {nat. }}^{(I I)}$ respectively. The subleading coefficients $g_{R, I}^{(n,-1)}$ and $g_{R, I I}^{(n,-1)}$ do not depend on the subtraction scale $\mu$ for $n>2$ to leading order in the $1 / m$ expansion. Therefore, at $\mu=0$ the coefficient $-g_{R, I I}^{(-2,-1)} / \mu_{\sigma}^{2}+g_{R, I I}^{(0,-1)}(0)$ and also $g_{R, I}^{(0,-1)}(0)$ may be anomalously small leading to an unnatural large scattering length $2 \pi a=-m T_{\left[{ }^{1} S_{0}\right]}(0)$. The required cancellation is achieved with an attractive coupling $\hat{g}_{R, I}^{(0,-1)}(0) \simeq \Lambda_{\text {nat. }}^{(I)} /\left(\pi f_{\pi}\right)$ in scenario I) and with $\hat{g}_{R, I I}^{(-2,-1)} \simeq-\mu_{\sigma}^{2} /\left(\pi f_{\pi} \Lambda_{\text {nat. }}^{(I I)}\right)$ in scenario II) where we identified $4 \pi f_{\pi} \simeq m$ for simplicity.

An anomalously large scattering length is evidence for the presence of nonperturbative structures at small momenta. If the precise singular structure is known one may proceed and break up and further expand the denominator of (25) without loosing accuracy in the expansion. Note, however, that such an 
expansion will necessarily be in conflict with unitarity. In scenario I) a systematic expansion of the denominator is induced by identifying $g_{R, I}^{(0,-1)}\left(m_{\pi}\right) \sim Q$ and $g_{R, I}^{(2,-1)}\left(m_{\pi}\right) p^{2} \sim Q^{2}$ with the typical small scale $Q$. This implies that the scattering amplitude $T_{\left[{ }^{1} S_{0}\right]}(p)$ shows either a shallow bound state or a pseudobound state $[1,15]$. The perturbative inclusion of pion dynamics according to the L-counting rule is straight forward. If the scattering amplitude $T_{\left[{ }^{1} S_{0}\right]}(p)$ exhibits a more complicated low-energy structure the scenario I) must be rejected as inefficient with an unnaturally small convergence radius. In scenario II) the relative importance of the two terms $g_{R, I I}^{(-2,-1)}$ and $g_{R, I I}^{(0,-1)}(\mu)$ depends crucially on the subtraction scale $\mu$. For small subtraction scale $\mu \sim m_{\pi}$, required for a manifest realization of the $L$-counting rule (13), both terms are equally important at $p \simeq 0$. In fact the low-energy characteristics of scenario II) is much richer than that of scenario I). At 'leading' orders the inverse scattering amplitude may have zeros at three different complex momenta corresponding to the roots of a cubic equation. It is unclear, in particular when the pion dynamics is included, in which way to expand the denominator without loosing the homogenous convergence property of (25). In scenario II) it is therefore advantageous to keep unitarity exactly and refrain from any further expansion beyond (25).

\subsection{S-wave scattering in the spin singlet channel}

Before evaluating $T_{\left[{ }^{1} S_{0}\right]}(p)$ to leading orders in the chiral expansion we recall its empirical behavior. A comparison of the structure of the empirical scattering amplitude with that of the amplitude in the two scenarios (25) provides strong indications to which scheme to choose.

The empirical scattering amplitude shows two striking phenomena: a pseudobound state close to threshold and a vanishing phase shift or equivalently a zero of the scattering amplitude $T_{\left[{ }^{1} S_{0}\right]}=0$ at $p \simeq 355 \mathrm{MeV}$. At very low energies with $E_{\text {lab }}<5 \mathrm{MeV}$ the partial wave amplitude $T_{\left[{ }^{1} S_{0}\right]}(p)$ is dominated by a pseudo-bound state pole

$$
\begin{aligned}
T_{\left[{ }^{1} S_{0}\right]}(p) & =\frac{8 \pi}{\sqrt{s}} \frac{1}{p \cot \delta\left({ }^{1} S_{0}\right)-i p} \\
& =\frac{4 \pi}{m}\left(\frac{i w}{p+i z}-b_{\mathrm{eff}}^{(0)}-i b_{\mathrm{eff}}^{(1)} p+\mathcal{O}\left(\frac{p^{2}}{m_{\pi}^{2}}\right)\right)
\end{aligned}
$$

where we made the pseudo-bound state pole contribution explicit and expanded the remainder in powers of $p$. The pole position $z$, its residuum, $w$, and the leading background parameters $b_{\text {eff }}^{(0,1)}$ are predicted accurately by the effective range theory 


$$
p \cot \delta=-\frac{1}{a}+\frac{1}{2} r p^{2}+\mathcal{O}\left(p^{4}\right)
$$

in terms of the s-wave np-scattering length $a \simeq-23.75 \mathrm{fm}$ and effective range parameter $r \simeq 2.75 \mathrm{fm}$. One finds

$$
\begin{aligned}
w^{-1} & =\sqrt{1-2 \frac{r}{a}} \simeq 0.90^{-1}, \quad z=\frac{1-w}{w r} \simeq 0.04 \mathrm{fm}^{-1}, \\
b_{\mathrm{eff}}^{(0)} & =a+\frac{r w^{2}}{1-w}=-\frac{w}{z} \frac{1-w}{1+w} \simeq-1.18 \mathrm{fm}, \\
b_{\mathrm{eff}}^{(1)} & =\frac{w}{z^{2}}-a^{2}=\frac{w}{z^{2}}\left(\frac{1-w}{1+w}\right)^{2} \simeq 1.53 \mathrm{fm}
\end{aligned}
$$

where we included also the prediction of the effective range theory for the next-to-leading moment in (27) for completeness. Note that for example the effective scattering length $b_{\text {eff }}^{(0)}$ is substantially suppressed as compared with the empirical scattering length $a \simeq-23.75 \mathrm{fm}^{4}$. We point out that the effective range formula (28) implies a second pole in the scattering amplitude at $p \simeq$ $i 0.77 / \mathrm{fm}$ :

$$
\begin{aligned}
& T_{\left[{ }^{1} S_{0}\right]}(p) \rightarrow \text { eff.range } \frac{8 \pi}{\sqrt{s}}\left(\frac{i w}{p+i z}-\frac{i w}{p-i \bar{z}}\right), \\
& \bar{z}=\frac{1+w}{1-w} z=\frac{1}{r}+\mathcal{O}\left(\frac{1}{a}\right) .
\end{aligned}
$$

From a theoretical point of view it is not obvious that the second pole at $p=i \bar{z}$ carries direct physical significance since it may 'move' as more terms are included in the expansion of the inverse $K$-matrix. In other words it could be an effective singularity which accounts for non-polynomial contributions from the exchange of pions. Note, however, that from scattering theory one expects that the analyticity domain of the s-wave amplitude is bounded due to the one-pion exchange contribution at $\Im p<m_{\pi} / 2$ and $m_{\pi} / 2<\bar{z}$ numerically.

We now discriminate two alternative scenarios. Suppose first that the strength of the pole at $p=i \bar{z}$ effectively accounts for perturbative pionic cuts. Then one may expand the amplitude as indicated in (27). The chiral theory is expected to replace the polynomial remainder in (27) by smooth and calculable analytic functions which exhibit the proper pionic cuts. A natural starting point for a theoretical description is scenario I) of (25) with the denominator in $T_{\left[{ }^{1} S_{0}\right]}^{(I)}(p)$ expanded according to the L-counting rule. This leads to a perturbative treatment of pions.

$\overline{4}$ It will turn out to be more convenient to work with the parameters $w$ and $z$. The scattering length $a$ and effective range are recovered as follows: $r=\frac{1-w}{w z}$ and $a=-\frac{2 w}{(w+1) z}$. 
Alternatively, suppose that the pole at $p=i \bar{z}$ has a physical significance in the sense that the exact solution of the problem shows an anomalously large subthreshold amplitude in the vicinity of $p \simeq i \bar{z}$. Then the expansion (27) turns useless at rather small momenta $p<\bar{z}$. Therefore an effective field theory description must incorporate that singularity properly. A promising

starting point is now scenario II) of (25). We expand the inverse of $T_{\left[{ }^{1} S_{0}\right]}^{(I)}$ and identify $\mu_{\sigma}$ with the empirical zero of the scattering phase. In this case we expect a significantly larger natural scale $\Lambda_{\text {nat. }}^{(I I)}>\mu_{\sigma} \simeq 350 \mathrm{MeV}$. In the next section we show in detail how the pion dynamics can be incorporated perturbatively according to the L-counting rule.

In this paper we will argue that the second scenario is in fact realized in nature. The success of the effective range theory, which describes the ${ }^{1} S_{0}$ phase accurately up to momenta $p \simeq 150 \mathrm{MeV}$, together with the L-counting rule already leads to the second scenario. Since the effective range theory can be viewed as an effective low energy theory where the pion degrees of freedom are integrated out, the second pole at $p \simeq i 150 \mathrm{MeV}$ points either at the importance of unitary two-pion exchanges with a branch point at $p=i m_{\pi}$ or at the physical significance of the second pole. On the other hand the chiral L-counting rule predicts that the pionic cut at $p=i m_{\pi}$ is weak. From the fact that the effective range theory works well up to surprisingly large momenta $p \simeq \bar{z} \simeq m_{\pi}$ and the effective range theory prediction of a strong second pole at $p=i \bar{z}$ with residuum $w \simeq 1$ (see (30)) we then conclude that we have choose the second scenario. This leads to an anomalously large subthreshold amplitude in the vicinity of $p \simeq i \bar{z}$ One arrives at a similar conclusion upon inspection of the typical size of the effective range parameter in both scenarios. Scenario II) yields a natural interpretation of the rather large empirical effective range parameter $1 / \bar{z} \sim r \simeq 2.75 \mathrm{fm}$ also in a model without pions. We return to this point in section 4 where we show that the empirical scattering amplitude can be rather nicely described by $T_{\left[{ }^{1} S_{0}\right]}^{(I)}(p)$ parameterized in terms of the four leading parameters in (25).

\subsection{Solving the Bethe-Salpeter equation}

In the previous section we derived a chiral power counting rule for appropriately subtracted 2-particle reducible diagrams generated by the once iterated Bethe-Salpeter kernel. In order to show that the counting rule (13) holds for more than one iteration of the Bethe-Salpeter kernel we now elaborate on how the subtraction scheme is implemented in the Bethe-Salpeter equation

$$
T=2 K+K G T
$$


The interaction kernel $K$ is the sum of polynomial contributions from 2nucleon contact interaction vertices, $K_{\sigma}$, which represents the exchange of heavy meson $(\sigma, \rho, \omega, \ldots)$ and the term $K_{\pi}$, which represents 2-particle irreducible pion exchange diagrams:

$$
\zeta=Y+\ldots=K_{\sigma}+K_{\pi} .
$$

Obviously the above separation of the interaction kernel $K$ is not unique since the irreducible multi-pion loops may also generate polynomial contact interaction terms. We will elaborate on this issue in detail below. Let us introduce the amplitude $T_{\pi}$

$$
T_{\pi}=2\left(1-K_{\pi} G\right)^{-1} K_{\pi}
$$

which is the solution of the Bethe-Salpeter equation for $K_{\sigma}=0$ and the auxiliary scattering amplitude $T_{\sigma}$ :

$$
\overbrace{1-T_{\sigma}}^{\left(1-K_{\sigma} G-\frac{1}{2} K_{\sigma} G T_{\pi} G\right)^{-1} K_{\sigma} .}
$$

The formal solution of the Bethe-Salpeter equation is easily expressed in terms of these amplitudes $T_{\sigma}$ and $T_{\pi}$

$$
C=\frac{1}{2}=T_{T_{\sigma}}+\frac{1}{4}
$$

Next we introduce a subtraction scheme for the Bethe-Salpeter equation. The subtracted kernels $K_{\pi, S}$ and $K_{\sigma, S}$ are defined in terms of an arbitrary and not yet specified subtraction interaction $K_{S}$

$$
\begin{aligned}
& K_{\pi, S}=K_{\pi}+K_{S}, \\
& K_{\sigma, S}=K_{\sigma}-K_{S} .
\end{aligned}
$$


According to our construction the full interaction kernel $K$ and consequently also the full scattering amplitude are manifestly independent of the subtraction interaction $K_{S}$

$$
K=K_{\pi}+K_{\sigma}=K_{\pi, S}+K_{\sigma, S}
$$

The subtracted interaction kernels induce the subtracted scattering amplitudes $T_{\pi, S}$ and $T_{\sigma, S}$ with:

$$
\begin{aligned}
& T_{\pi, S}\left[K_{S}\right]=2\left(1-K_{\pi, S} G\right)^{-1} K_{\pi, S}, \\
& T_{\sigma, S}\left[K_{S}\right]=2\left(1-K_{\sigma, S} G-\frac{1}{2} K_{\sigma, S} G T_{\pi, S}\left[K_{S}\right] G\right)^{-1} K_{\sigma, S} .
\end{aligned}
$$

The full scattering amplitude remains formally invariant

$$
T=T_{\pi, S}\left[K_{S}\right]+\left(1+\frac{1}{2} T_{\pi, S}\left[K_{S}\right] G\right) T_{\sigma, S}\left[K_{S}\right]\left(1+\frac{1}{2} G T_{\pi, S}\left[K_{S}\right]\right)
$$

One is now free to exploit the choice of the subtraction interaction $K_{S}$. Different choices for $K_{S}$ may increase or decrease the average interaction strength in $K_{\pi, S}$. Therefore one would expect that the non-perturbative part of the scattering amplitude like for example the deuteron bound state may sit either in $T_{\sigma, S}$ or $T_{\pi, S}$ depending on the choice of $K_{S}$. Suppose now that non-perturbative effects sit in $T_{\sigma, S}$ then one may conclude that the amplitude $T_{\pi, S}$ is perturbative and can be expanded in powers of $K_{\pi, S}$. We point out that in this case also the amplitude $T_{\sigma, S}$ need not to be evaluated exactly. The perturbative character of the subtracted pion iteration permits the expansion of the inverse amplitude $T_{\sigma, S}$ in powers of the kernel $K_{\pi, S}$.

$$
\begin{aligned}
T_{\sigma, S} & =2\left(1-K_{\sigma, S} G-K_{\sigma, S} G K_{\pi, S} G+\ldots\right)^{-1} K_{\sigma, S} \\
& =2\left(K_{\sigma, S}^{-1}-G-G K_{\pi, S} G+\ldots\right)^{-1} .
\end{aligned}
$$

Here we refrain form breaking up and further expanding the denominator in (40) since that requires further assumptions on the structures of nonperturbative effects in $T_{\sigma, S}$.

It is useful to outline our renormalization procedure. At given chiral order $n$, construct the local kernel $K_{S}=\bar{K}_{S}$ perturbatively such that

$$
T_{\pi, S}\left[\bar{K}_{S}\right]=\text { finite }+\mathcal{O}\left(Q^{n+1}\right) \quad \& \quad T_{\pi, S}\left[\bar{K}_{S}\right] G=\text { finite }+\mathcal{O}\left(Q^{n+2}\right)
$$


holds. Note that $T_{\pi, S} G$ can be defined via $T_{\pi, S} G K_{\sigma, S}$ if $K_{\sigma, S}$ is separable. We emphasize that the construction (41) differs from conventional renormalization procedures insofar that the object $T_{\pi, S} G$ is rendered finite by 'counter loops' rather then counter terms. In particular $T_{\pi, S}$ and $T_{\pi, S} G$ do not depend on any renormalization scale. As an immediate consequence note that for $T_{\pi, S}$ and also the vertex loop functions $T_{\pi, S} G$ the L-counting rule apply without a further subtraction procedure (see (12)). The only remaining divergencies sit in $T_{\sigma, S}$ and can be absorbed into the local bare coupling $K_{\sigma}$ :

$$
K_{\sigma, S}^{-1}-G-G T_{\pi, S}\left[\bar{K}_{S}\right] G=\text { finite }+\mathcal{O}\left(Q^{n+3}\right)
$$

Note that the divergencies in $K_{\sigma, S} G T_{\pi, S} G K_{\sigma, S}$ are 'weak' since they are already tamed to a large extend by the renormalization condition (41). Here a manifest realization of the L-counting rule requires a subtraction scale $\mu \sim m_{\pi}$.

It is not obvious that the renormalization conditions (41) and (42) can be met. In fact our scheme requires that the Bethe-Salpeter interaction kernel $K$ is 'onshell irreducible' if we insist on a separable counter interaction $K_{S}$. Basically we need a mechanism which absorbs systematically all divergencies not directly linked to a s-channel unitarity cut into $K_{\sigma}$ at the level of the interaction kernel. We decompose the kernel $K$ of (31) into an 'on-shell irreducible' part $\bar{K}$ and 'on-shell reducible' terms $K_{L}, K_{R}$ and $K_{L R}$ which vanish if evaluated with on-shell kinematics either in the incoming or outgoing channel

$$
K=\bar{K}+K_{L}+K_{R}+K_{L R}
$$

Here $K_{R}\left(K_{L}\right)$ vanishes at on-shell kinematics for the incoming (outgoing) nucleons after multiplication with the incoming (outgoing) nucleon spinors by construction. The term $K_{L R}$ vanishes if evaluated with either incoming or outgoing on-shell kinematics. For example the s-channel spectral density of $K_{R} G \bar{K}$ or $\bar{K} G K_{L}$ is zero. A term like $K_{R} G$ would be renormalized according to (41) by an appropriate counter loop $K_{S} G$ with a non-separable $K_{S}$. This would destroy the simple structure of $T_{\sigma, S}$. It is therefore advantageous to eliminate all on-shell reducible terms by introducing the on-shell equivalent effective interaction kernel $\hat{K}$ with

$$
\begin{aligned}
& \hat{T}=2 \hat{K}+\hat{K} G \hat{T}=\left.\frac{2}{1-K G} K\right|_{\text {on-shell }}, \\
& \hat{K}=\bar{K}+K_{R} G \bar{K}+\bar{K} G K_{L}+K_{R} G K_{L}+\bar{K} G K_{L R} G \bar{K}+\cdots
\end{aligned}
$$

By construction the effective interaction kernel $\hat{K}$ is on-shell irreducible and one therefore expects a non-zero s-channel spectral density of $\hat{K} G \hat{K}$. We point out that it is sufficient to work out the matching in (44) perturbatively since 
chiral power counting rules apply for $\hat{K}^{5}$. In full analogy to (37) the effective kernel $\hat{K}=\hat{K}_{\pi}+\hat{K}_{\sigma}$ is written as a sum of non-local contributions $\hat{K}_{\pi}$ and local counter terms $\hat{K}_{\sigma}$. Divergent terms in $\hat{K}$ can now be absorbed into the local interaction vertex $\hat{K}_{\sigma}$ of (32) at the level of the interaction kernel.

We need to address a further aspect of our scheme: how does one renormalize divergencies that appear in subleading orders in the $1 / m$ expansion. To this end it is convenient to introduce a partly renormalized two-nucleon propagator $\tilde{G}$ :

$$
\begin{aligned}
& \tilde{G}(l ; w)=\int_{4 m^{2}}^{4\left(m^{2}+\Lambda^{2}\right)} \frac{d \bar{s}}{\pi} \sqrt{\frac{\bar{s}}{w^{2}}} \frac{-1}{\bar{s}-w^{2}-i \epsilon} \Delta S_{F}^{(+)}(l, w ; \bar{s}) \otimes \Delta S_{F}^{(-)}(l, w ; \bar{s}), \\
& \Delta S_{F}^{( \pm)}(l, w ; \bar{s})=i \pi \delta\left(l^{2} \pm l \cdot w+\frac{1}{4} \bar{s}-m^{2}\right)\left( \pm l+\frac{1}{2} \psi+m\right)
\end{aligned}
$$

where we keep the cutoff parameter $\Lambda$ at this intermediate stage. Replacing the two-nucleon propagator $G$ by the renormalized propagator $\tilde{G}$ leads to a renormalized interaction kernel $\tilde{K}$

$$
\hat{T}=2 \hat{K}+\hat{K} G \hat{T}=2 \tilde{K}+\tilde{K} \tilde{G} \hat{T}, \quad \tilde{K}=\frac{1}{1-\hat{K}(G-\tilde{G})} \hat{K}
$$

It is crucial to observe that by construction $\tilde{K}$ is free of s-channel unitarity cuts. Therefore $\tilde{K}$ can be evaluated in perturbation theory applying chiral power counting rules. The construction (46) defines a systematic prescription for absorbing ill-defined power divergent terms $(\Lambda / m)^{n}$ with $n>1$ induced by the $1 / m$ expansion into $K_{\sigma}$ (see $(20)$ ). Note that for a local vertex (14) we would find $\tilde{K}=\hat{K}$ if we replaced $\sqrt{\bar{s} / w^{2}} \rightarrow 1$ in (45).

In the following we assume that the kernel $K$ in (31) is on-shell irreducible and $1 / m$ renormalized according to $(44,46)$. We emphasize that the constructions (44) and (46) are needed to systematically absorb a large class of divergencies into the local interaction kernel $K_{\sigma}$. Only after the effective interaction kernel has been properly identified, can one apply the renormalization conditions $(41,42)$. Note that in actual calculations it is not mandatory to construct the effective interaction explicitly. Since the pion-dynamics at given chiral order leads to a finite number of Feynman diagrams to be considered it is sufficient to evaluate those diagrams in terms of the original bare interaction kernel

5 Note that the introduction of the on-shell irreducible kernel via (44) demonstrates that the infinite tower of local s-wave interaction terms in (14) is effectively complete. Further s-wave interaction terms simply renormalize the coupling function of (14) according to (44). 
$K$. The construction (44) and(46) then leads to a prescription for moving divergencies, which are not absorbed with $(41,42)$, into $K_{\sigma}$ (see appendix B).

We turn to a problem with the proposed expansion: as it stands it violates unitarity. Here we anticipate an important result to be discussed in detail below, namely that such an expansion can be reconciled with unitarity if the subtraction interaction $K_{S}$ is generalized to allow for more complicated separable structures. Rather than evaluating for example $T_{\pi, S}\left[K_{S}\right]$ strictly at $K_{S}=\bar{K}_{S}$ as suggested by the renormalization condition (41) we consider $T_{\pi, S}\left[K_{S}\right]$ at $K_{S} \neq \bar{K}_{S}$. It is crucial not to spoil the renormalization procedure $(41,42)$. Therefore the non-trivial part of $K_{S}$ is permitted only in the finite parts. More specifically we split for example $T_{\pi, S}\left[K_{S}\right]$ in a finite part $T_{\pi, S}^{f i n .}\left[K_{S}\right]$ and a divergent part $T_{\pi, S}^{d i v}$. $\left[K_{S}\right]$ and expand in $K_{S}$ around $\bar{K}_{S}$ :

$$
\begin{aligned}
T_{\pi, S}\left[p^{2} ; K_{S}\right] & =T_{\pi, S}^{f i n .}\left[p^{2}, \bar{\mu}^{2} ; K_{S}\right]+T_{\pi, S}^{d i v} \cdot\left[p^{2}, \bar{\mu}^{2} ; K_{S}\right] \\
& =\sum_{n=0}^{\infty}\left(T_{\pi, d i v .}^{(n)}\left(p^{2}, \bar{\mu}^{2}\right)+T_{\pi, f i n .}^{(n)}\left(p^{2}, \bar{\mu}^{2}\right)\right)\left(K_{S}-\bar{K}_{S}\right)^{n}, \\
T_{\pi, \text { fin. }}^{(n)}\left(-\bar{\mu}^{2}, \bar{\mu}^{2}\right) & =0 \quad \text { if } T_{\pi, d i v .}^{(n)}\left(p^{2}, \bar{\mu}^{2}\right) \neq 0
\end{aligned}
$$

where we introduce a necessary splitting point $\bar{\mu}$. In (47) the local kernel $\bar{K}_{S}$ is fixed according to the renormalization condition (41). We implicitly assume a projection onto a given partial wave. Thus the scattering amplitude is a function of only the relative momentum $p$ with $s=4\left(m^{2}+p^{2}\right)$. Note for example that by construction $T_{\pi, d i v .}^{(0)}\left(p^{2}, \bar{\mu}^{2}\right)=0$ and therefore $T_{\pi, f i n .}^{(n)}\left(p^{2}, \bar{\mu}^{2}\right)$ is independent of the splitting point $\bar{\mu}$. Moreover in (47) we assume that the nontrivial part of the kernel $K_{S}$ is separable since only then the multiple powers of $\left(K_{S}-\bar{K}_{S}\right)^{n}$ are defined properly. The separation of the objects $T_{\pi, S} G$ and $G T_{\pi, S}$ into finite and divergent parts is performed in complete analogy with (47). The remaining objects $G T_{\pi, S} G$ and $K_{\sigma, S}$ require a separate treatment since they involve further divergencies which are not taken care of by $\bar{K}_{S}$ :

$$
\begin{aligned}
K_{\sigma, S}^{-1}-G & =G T_{\pi, S}\left[K_{S}\right] G \\
& +\sum_{n=0}^{\infty}\left(T_{\sigma, d i v .}^{(n,-1)}\left(p^{2}, \bar{\mu}^{2}\right)+T_{\sigma, \text { fin. }}^{(n,-1)}\left(p^{2}, \bar{\mu}^{2}\right)\right)\left(K_{S}-\bar{K}_{S}\right)^{n}, \\
T_{\sigma, \text { fin. }}^{(n,-1)}\left(-\bar{\mu}^{2}, \bar{\mu}^{2}\right)= & \text { if } T_{\sigma, \text { div. }}^{(n,-1)}\left(p^{2}, \bar{\mu}^{2}\right) \neq 0 .
\end{aligned}
$$

Again by construction $T_{\sigma, d i v .}^{(0,-1)}\left(p^{2}, \bar{\mu}^{2}\right)=0$ and therefore $T_{\sigma, \text { fin. }}^{(0,-1)}\left(p^{2}, \bar{\mu}^{2}\right)$ is independent on the splitting point $\bar{\mu}$. Note that the splitting procedure in (47) and (48) must necessarily be correlated in order to ensure that the full scattering amplitude $T$ is independent of $K_{S}$ and thus on any power $\left(K_{S}-\bar{K}_{S}\right)^{n}$ as it should be according to our construction $(36,37)$. The independence of $T$ on $K_{S}-\bar{K}_{S}$ serves as a powerful argument that all divergent terms like 
$T_{\pi, d i v .}^{(n)}\left(p^{2}, \bar{\mu}^{2}\right)\left(K_{S}-\bar{K}_{S}\right)^{n}$ can be dropped. Note that any truncation of $T_{\pi, S}$ and $T_{\sigma, S}$ of the form (40) necessarily leads to a residual dependence of $T$ on $K_{S}$. This dependence of the approximate $T$ on $K_{S}-\bar{K}_{S}$ can then be exploited to restore unitarity.

We proceed and present an argument that the splitting point $\bar{\mu}^{2}$ must in fact be identified with the chiral limit value of a potential singularity present in $T$ at $p^{2}=-z_{0}^{2}=-\bar{\mu}^{2}$ in a given partial wave ${ }^{6}$. Suppose we drop the nontrivial terms $\left(K_{S}-\bar{K}_{S}\right)^{n}$ for a moment since in the full expression for $T$ they cancels anyway. Then we expect the singularity to sit in $T_{\sigma, \text { fin. }}^{(0,-1)}\left(-z_{0}^{2}\right)=0$ since $T_{\pi, f i n .}^{(0)}\left(p^{2}\right)$ is perturbative and independent on $\bar{\mu}$ by construction. Therefore, if we now include the terms $\left(K_{S}-\bar{K}_{S}\right)^{n}$, we must insist on $T_{\sigma, f i n .}^{(n,-1)}\left(-z_{0}^{2}, \bar{\mu}^{2}\right)=$ 0 also for $n>0$. If we do not insist on this condition the singularity at $p^{2}=-z_{0}^{2}$ must sit in $T_{\pi, f i n}^{(0)}$. which contradicts our construction. Here we implicitly assume that the non-trivial part in $K_{S}$ is also perturbative. This will be confirmed later when this part is constructed explicitly. We now arrive at our result $\bar{\mu}^{2}=z_{0}^{2}$ since from (47) it follows that $T_{\sigma, \text { div. }}^{(n>0,-1)}\left(p^{2}, \bar{\mu}^{2}\right) \neq 0$ holds.

We emphasize that the local part of $\bar{K}_{S}$ is fixed by the renormalization condition (41) whereas the non-trivial terms $\left(K_{S}-\bar{K}_{S}\right)^{n}$, which are included exclusively in the finite parts, are determined by the unitarity constraint. In our discussion of the separation of finite parts from infinite parts, we suppressed the chiral expansion of e.g. $K_{S}$ and $T_{\pi, S}$. It is implicitly understood that $(47,48)$ apply order by order in the chiral expansion.

On a rather formal level the underlying mechanism leading to exact unitarity is quickly revealed. At given chiral order $n$ construct the non-trivial but separable part of $K_{S}$ such that

$$
\left\langle T_{\pi, S}\right\rangle=0+\mathcal{O}\left(Q^{n+1}\right)
$$

holds, where the brackets refers to projection onto a given partial wave with on-shell kinematics. Insisting on a separable construction of $K_{S}$ leads to the crucial identities:

$$
\begin{aligned}
\langle T\rangle & =2 \frac{\left\langle 1+\frac{1}{2} T_{\pi, S} G\right\rangle\left\langle 1+\frac{1}{2} G T_{\pi, S}\right\rangle+\mathcal{O}\left(Q^{n+2}\right)}{\left\langle K_{\sigma, S}^{-1}-G-\frac{1}{2} G T_{\pi, S} G\right\rangle+\mathcal{O}\left(Q^{n+3}\right)}, \\
0 & =\Im\left\langle 1+\frac{1}{2} T_{\pi, S} G\right\rangle\left\langle 1+\frac{1}{2} G T_{\pi, S}\right\rangle+\mathcal{O}\left(Q^{n+2}\right) .
\end{aligned}
$$

${ }^{6}$ In the spin triplet channel for example we would identify $z_{0}=\sqrt{2 m \epsilon_{D}-\epsilon_{D}^{2}}$ with the nucleon mass $m$ and the deuteron binding energy $\epsilon_{D}$ evaluated in the chiral limit with $m_{\pi}=0$. 
The unitarity condition

$$
\Im T^{-1}=-\frac{1}{2} \Im G
$$

applied to (39) reduces to:

$$
\Im\left\langle G+\frac{1}{2} G T_{\pi, S} G\right\rangle=(\Im\langle G\rangle)\left(\Re\left\langle 1+\frac{1}{2} T_{\pi, S} G\right\rangle\left\langle 1+\frac{1}{2} G T_{\pi, S}\right\rangle\right) .
$$

The unitarity condition (52) is indeed fulfilled if expanded according to chiral power counting rules. This is basically Cutkosky's cutting rule [23]. The explicit technical realization of this mechanism will be presented in section 3.4 .

According to the chiral counting rules one expects that any non-perturbative structure must sit in the denominator of (50). Thus any attempt to break up and expand further this denominator is subtle if the precise singularity structure is unknown. We suggest a scheme in which the numerator and denominator of (50) are expanded according to the counting rule (13) separately. This expansion has the great advantage that it respects unitarity exactly order by order in the chiral expansion. Note that if $T_{\pi, S}=0$ the full solution of the Bethe-Salpeter equation is represented by $T_{\sigma, S}$. In this sense one may say that unitarity leads to an optimized chiral expansion of the scattering amplitude. We stress that a perturbative $K_{\pi, S}$ kernel is predicted by chiral power counting rules provided that the subtraction interaction $\bar{K}_{S}$ is constructed properly. In particular all divergencies must be absorbed in $T_{\pi, S}$ and $T_{\pi, S} G$. A positive feature of this scheme is its practicality: the object $T_{\pi, S}$ can be evaluated perturbatively and therefore, due to the separable nature of $K_{S}$, the scattering amplitude can be evaluated analytically.

\section{The nucleon-nucleon scattering amplitude}

In this section we derive explicit expressions for the leading chiral orders of the nucleon-nucleon scattering amplitude constructed according to the chiral counting scheme of section 2. We will present analytic results for the required loop functions and confirm the usefulness of our L-counting rule.

We parameterize the on-shell scattering amplitude, $T(t, u)$, in terms of five

isospin zero amplitudes, $a_{i}^{(0)}(t, u)$, and five isospin one amplitudes, $a_{i}^{(1)}(t, u)$,

$$
T(t, u)=\mathcal{P}_{0} \sum_{i=0}^{5} a_{i}^{(0)}(t, u) T_{i}+\mathcal{P}_{1} \sum_{i=0}^{5} a_{i}^{(1)}(t, u) T_{i}
$$


defined with respect to the Dirac tensors

$$
\begin{aligned}
& T_{1}=\left(\gamma_{5} C^{-1} \times C \gamma_{5}\right), \\
& T_{2}=\left(\gamma_{\alpha} C^{-1} \times C \gamma^{\alpha}\right) \\
& T_{3}=\left(C^{-1} \times C\right) \\
& T_{4}=\left(\gamma_{\alpha} C^{-1} \times C \gamma^{\alpha}\right)-\frac{1}{2}\left(\sigma_{\alpha \beta} C^{-1} \times C \sigma^{\alpha \beta}\right), \\
& T_{5}=\left(\gamma_{5} C^{-1} \times C \gamma_{5}\right)+\left(\gamma_{5} \gamma_{\alpha} C^{-1} \times C \gamma_{5} \gamma^{\alpha}\right)
\end{aligned}
$$

with the charge conjugation matrix $C=i \gamma_{0} \gamma_{2}$ and the isospin projection operators

$$
\begin{aligned}
& \mathcal{P}_{0}=\frac{1}{2}\left(\tau_{2} \times \tau_{2}\right), \\
& \mathcal{P}_{1}=\frac{1}{2}\left(\vec{\tau} \tau_{2} \times \tau_{2} \vec{\tau}\right)
\end{aligned}
$$

For the precise definition of the tensor product $\times$ we refer to appendix A. The invariant amplitudes, $a_{i}^{(I)}(t, u)$, depend on the Mandelstam variables $t=$ $\left(p_{1}-p_{1}^{\prime}\right)^{2}$ and $u=\left(p_{1}-p_{2}^{\prime}\right)^{2}$ where $p_{1,2}$ are the 4 -momenta of the incoming nucleons and $p_{1,2}^{\prime}$ those of the outgoing nucleons. The Dirac tensors $T_{i}$ are constructed such that they are invariant modulo a sign upon flipping incoming or outgoing indices:

$$
\left(T_{i}\right)_{k l m n}=(-1)^{i}\left(T_{i}\right)_{k l n m}=(-1)^{i}\left(T_{i}\right)_{l k m n}
$$

As a consequence one finds also a simple transformation property of the reduced amplitudes, $a_{i}^{(I)}(u, t)=(-1)^{i+I} a_{i}^{(I)}(t, u)$, under the interchange of incoming or the outgoing particles. Furthermore the tensors $T_{i}$ are chosen such that the $T_{i \geq 3}$ are kinematically suppressed by one power of $u$ or $t$ if multiplied with on-shell spinors. In appendix A the invariant amplitudes $a_{i}^{(I)}(t, u)$ are uniquely reconstructed in terms of the nucleon phase shifts.

In this work we focus on the isospin one channel and evaluate the $a_{i}^{(1)}(u, t)$ amplitudes to leading orders in our chiral expansion scheme. Results on the isospin zero amplitudes will be reported on in a forthcoming publication. Here we elaborate in particular on the s-wave partial wave amplitude $T_{\left[{ }^{1} S_{0}\right]}(p)$ which is parameterized uniquely in terms of the s-wave phase shift $\delta_{\left[{ }^{1} S_{0}\right]}(p)$. The swave amplitude can be constructed in terms of our reduced amplitudes

$$
T_{\left[{ }^{1} S_{0}\right]}(p)=\int \frac{d \Omega}{4 \pi} T_{\left[{ }^{1} S_{0}\right]}(u, t)=\frac{4 \pi}{\sqrt{s}} \frac{1}{i p}\left(e^{2 i \delta_{\left[{ }^{1} S_{0}\right]}}-1\right)
$$

where 


$$
T_{\left[{ }^{1} S_{0}\right]}(u, t)=a_{1}^{(1)}(u, t)-\frac{u-t}{s} a_{4}^{(1)}(u, t)-\frac{u+t}{s} a_{5}^{(1)}(u, t)
$$

and $t=-2 p^{2}(1-\cos \theta), u=-2 p^{2}(1+\cos \theta)($ see $(\mathrm{A} .5, \mathrm{~A} .8))$.

We proceed and derive the leading terms of the scattering amplitude (39). The central interaction vertex relevant for our work is the pion-nucleon coupling vertex extracted from the chiral Lagrangian

$$
\mathcal{L}=g_{A} \lambda \bar{N} \gamma_{5} \gamma_{\mu}\left(\partial^{\mu} \vec{\pi} \cdot \vec{\tau}\right) N=\frac{g_{A} m}{f_{\pi}} \bar{N} i \gamma_{5} \vec{\pi} \cdot \vec{\tau} N+\cdots
$$

where the coupling constant $\lambda$ is identified with the pion decay constant $\lambda^{-1}=$ $2 f_{\pi} \simeq 186 \mathrm{MeV}$. For the axial vector coupling constant of the nucleon we take $g_{A} \simeq 1.26$. To the leading orders we will be working here the interaction kernel $K_{\pi}$ of $(32)$ is identified with the one-pion exchange contribution

$$
K_{\pi}=-\frac{g_{A}^{2}}{8 f_{\pi}^{2}} \frac{\gamma_{5} \phi \otimes \gamma_{5} \phi}{t-m_{\pi}^{2}}\left(\mathcal{P}_{1}-3 \mathcal{P}_{0}\right)+\text { exchange term }
$$

following from (59) and $Q=p_{1}-p_{1}^{\prime}$. The on-shell irreducible kernel $\hat{K}_{\pi}$ of (44) follows with

$$
\begin{aligned}
\hat{K}_{\pi} & =\frac{g_{A}^{2} m^{2}}{2 f_{\pi}^{2}} \frac{\gamma_{5} \otimes \gamma_{5}}{t-m_{\pi}^{2}}\left(\mathcal{P}_{1}-3 \mathcal{P}_{0}\right) \\
& -\frac{g_{A}^{4} m^{2}}{16 f_{\pi}^{4}}\left(2\left(m_{\pi}^{2}-\frac{t}{2}\right) I_{3, \pi}(t)+I_{2, \pi}(t)-2 I_{2}\left(m^{2}\right)\right)\left(T_{1}+T_{2}\right)\left(\mathcal{P}_{1}+9 \mathcal{P}_{1}\right) \\
& -\frac{11 g_{A}^{4} m^{2}}{384 f_{\pi}^{4}} I_{1, \pi}\left(T_{1}+T_{2}\right)\left(\mathcal{P}_{1}+9 \mathcal{P}_{1}\right)+\mathcal{O}\left(Q^{2}\right)+\text { exchange terms }(61)
\end{aligned}
$$

At leading orders it is given by the pion-nucleon vertex with pseudo-scalar coupling and contributions from scalar one-loop functions $I_{3, \pi}(t), I_{2, \pi}(t), I_{1, \pi}$ and $I_{2}\left(m^{2}\right)$ defined in appendix B. All we would like to point out here is that we find:

$$
\left.\left(K_{\pi}+K_{\pi} G K_{\pi}\right)\right|_{\text {on-shell }}=\hat{K}_{\pi}+\hat{K}_{\pi} G \hat{K}_{\pi}+\mathcal{O}\left(Q^{2}\right)
$$

by construction. Our result (61) clearly exemplifies the merit of transforming to the on-shell irreducible kernel: it permits to move the divergent pionic tadpole contribution $I_{1, \pi}$ into the local interaction vertex $K_{\sigma}$ of (32) at the level of the interaction kernel. An interesting observation worked out in detail in appendix B.1 concerns the chiral power of the pionic tadpole diagram $I_{1, \pi}$. As is demonstrated explicitly a consistent $1 / m$ expansion implies $I_{1, \pi} \sim Q$ rather 
than $I_{1, \pi} \sim Q^{2}$ as one might expect naively from (3). Here this does not cause any complication since $I_{1, \pi}$ is absorbed into the bare coupling function. Note a further subtle property of the on-shell irreducible kernel $\hat{K}_{\pi}$ : the scalar pion bubble loop $I_{2, \pi}(t)$ in (61), carries chiral power zero (see (B.10)). This does not contradict the chiral expansion since this term is canceled by a corresponding term in $\hat{K}_{\pi} G \hat{K}_{\pi}$ by construction.

The kernel $K_{\sigma}$ was already given in (15) in terms of the energy dependent coupling function $g(s)$. It is on-shell irreducible by construction. We further need to specify the subtraction interaction $K_{S}$ of (36) and $\bar{K}_{S}$ of (41)

$$
\begin{gathered}
K_{S}=c(s) g_{A}^{2} \lambda^{2} T_{1} \mathcal{P}_{1}, \bar{K}_{S}=\bar{c}(s) g_{A}^{2} \lambda^{2} T_{1} \mathcal{P}_{1}, \\
K_{\sigma, S}=g_{\sigma, S}(s) \\
=\left(g(s)-c(s) g_{A}^{2} \lambda^{2}\right) T_{1} \mathcal{P}_{1}
\end{gathered}
$$

appropriate for s-wave scattering in the spin singlet channel. Here we allow for a s-dependence of the subtraction function $c(s)$. The subtraction function $c(s)$ is on the one hand required to justify our previously introduced L-counting rule and on the other hand ultimately leads to unitary results for the scattering amplitude.

The leading orders as they contribute to our amplitudes are readily derived. The amplitude $T_{\pi, S}(s)$ of (38) receives at chiral order $Q$ contributions

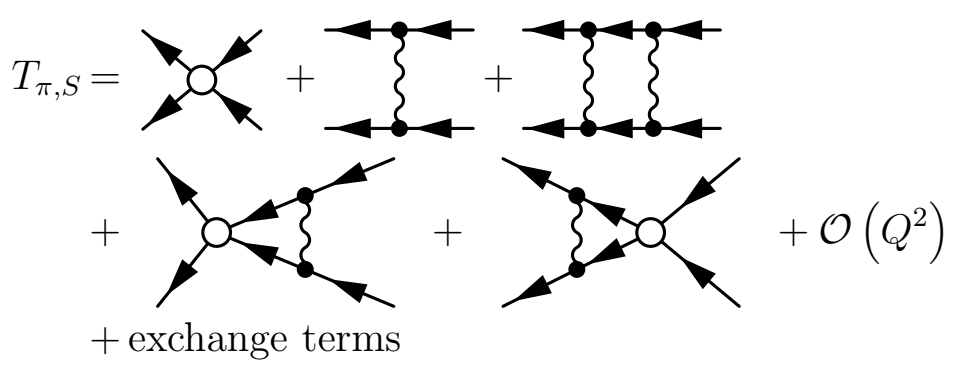

from the subtraction vertex $K_{S}$, the one-pion exchange, the pion nucleon box and triangle diagrams induced by $K_{S}$. In (64) the wavy line represents the pion exchange $K_{\pi}$ of (60) rather than $\hat{K}_{\pi}$ of (61) for convenience. Transforming back to the on-shell irreducible kernel $\hat{K}_{\pi}$ is straightforward and only required for the renormalization process. The full scattering amplitude follows now from (39). We note that all reduced amplitudes $a_{i}^{(1)}(u, t)$ receive contributions from $T_{\pi, S}$ whereas the amplitude $T_{\sigma, S}$ affects only $a_{1}^{(1)}(u, t)$ :

$$
\begin{aligned}
a_{1}^{(1)}(u, t) & =2 g_{A}^{2} \lambda^{2} c(s)\left(1+2 g_{A}^{2} \lambda^{2} V_{\pi}(s)\right)+2 g_{A}^{4} \lambda^{4} c^{2}(s) J(s) \\
& +g_{A}^{4} \lambda^{4}\left(B_{1}(u, t)+B_{1}(t, u)\right)
\end{aligned}
$$




$$
\begin{aligned}
& +\left(1+g_{A}^{2} \lambda^{2} V_{\pi, S}(s)+g_{A}^{4} \lambda^{4} V_{\pi \pi, S}(s)\right)^{2} T_{\sigma, S}(s), \\
a_{2}^{(1)}(u, t) & =g_{A}^{4} \lambda^{4}\left(B_{2}(u, t)-B_{2}(t, u)\right), \\
a_{3}^{(1)}(u, t) & =m^{2} g_{A}^{2} \lambda^{2}\left(\frac{1}{t-m_{\pi}^{2}}+\frac{1}{u-m_{\pi}^{2}}\right)+g_{A}^{4} \lambda^{4}\left(B_{3}(u, t)+B_{3}(t, u)\right), \\
a_{4}^{(1)}(u, t) & =m^{2} g_{A}^{2} \lambda^{2}\left(\frac{1}{u-m_{\pi}^{2}}-\frac{1}{t-m_{\pi}^{2}}\right)+g_{A}^{4} \lambda^{4}\left(B_{4}(u, t)-B_{4}(t, u)\right), \\
a_{5}^{(1)}(u, t) & =m^{2} g_{A}^{2} \lambda^{2}\left(\frac{1}{t-m_{\pi}^{2}}+\frac{1}{u-m_{\pi}^{2}}\right)+g_{A}^{4} \lambda^{4}\left(B_{5}(u, t)+B_{5}(t, u)\right) .
\end{aligned}
$$

The functions $B_{i}(u, t)$ represent the contribution from the pion-nucleon box diagram in (64). The precise definition for all loop functions can be found in appendix B. The one-pion exchange contribution is made explicit in (65) and confirms the transformation property of the amplitudes $a_{i}(t, u)$ under interchange of $t$ and $u$. The vertex loop functions $V_{\pi^{n}, S}(s)$ in (65) are introduced with respect to the interaction kernel $K_{\pi, S}$ of (36). The one and two-loop vertex functions $V_{\pi, S}(s)$ and $V_{\pi, S}(s)$ are then given by

$$
\begin{aligned}
& V_{\pi, S}(s)=\frac{1}{g_{A}^{2} \lambda^{2}} \\
& =V_{\pi}(s)+c(s)\left(J(s) V_{\pi}(s)+J_{\pi}(s)\right)+(s)
\end{aligned}
$$

in terms of the vertex functions $V_{\pi}(s)$ and $V_{\pi \pi}(s)$ defined with respect to the unsubtracted interaction kernel $K_{\pi}$ of $(60)$, the not yet specified subtraction function $c(s)$ and the one-loop bubble function $J(s)$ introduced in (16). The pole term in $(65)$

$$
T_{\sigma, S}(s)=\frac{2}{g_{\sigma, S}^{-1}(s)-J(s)-g_{A}^{2} \lambda^{2} J_{\pi, S}(s)-g_{A}^{4} \lambda^{4} J_{\pi \pi, S}(s)+\ldots}
$$

is determined by the bubble functions $J_{\pi^{n}, S}(s)$ introduced with respect to 
the interaction kernel $K_{\pi, S}$ of (36). Again the subtracted two and three-loop bubble integrals $J_{\pi, S}(s)$ and $J_{\pi \pi, S}(s)$ can be expressed as follows

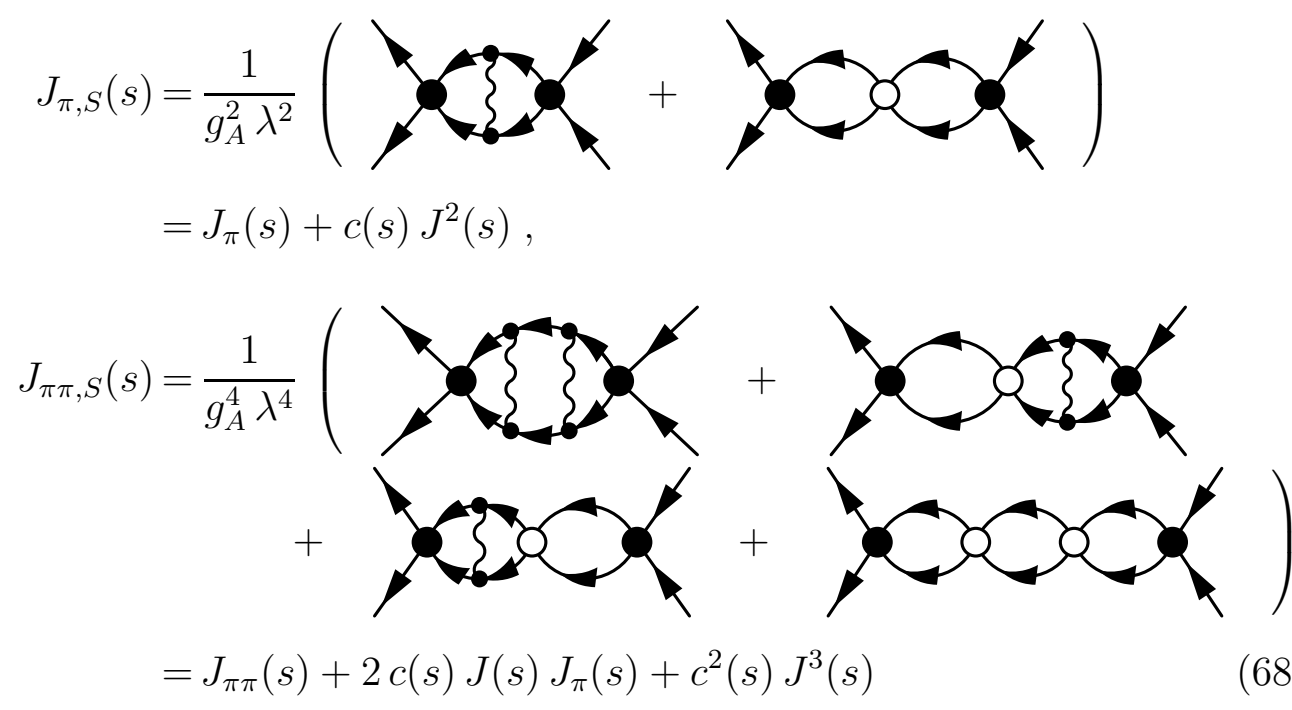

where $J_{\pi}(s)$ and $J_{\pi \pi}(s)$ are the 'unsubtracted' loop functions.

We will demonstrate that our L-counting rule can be applied for the loop functions

$$
V_{\pi^{n}, S}(s) \sim Q^{n}, \quad J_{\pi^{n}, S}(s)-J_{\pi^{n}, S}\left(s_{0}\right) \sim Q^{n+1}
$$

provided that $c=\bar{c}$ with $\bar{c}=1 / 2+\mathcal{O}\left(Q^{2}\right)$ and the subtraction point $s_{0} \sim 4 m^{2}$ sufficiently close to threshold. According to the L-counting rule the leading chiral power of the vertex functions $V_{\pi^{n}, S}(s)$ and bubble functions $J_{\pi^{n}, S}(s)-$ $J_{\pi^{n}, S}\left(s_{0}\right)$ is given by the number of independent loops. We return to the chiral powers of these loop functions in more detail below when we present explicit analytic results for all loop functions introduced here. For more details on the evaluation of the loop functions we refer the reader to appendix B.

We proceed and systematically rewrite the bare loop functions $J(p), J_{\pi, S}(p)$ and $V_{\pi, S}(p)$ of (65) and (67) in terms of renormalized loop functions $J_{R}\left(p, z_{0}\right)$, $J_{\pi, R}\left(p, z_{0}\right)$ and $V_{\pi, R}(p)$. Note that from now on we consider all loop functions as a function of the small momentum $p$ rather than the Mandelstam variable $s=4\left(m^{2}+p^{2}\right)$. For simplicity we consider in this section only the leading term in the $1 / m$ expansion and systematically drop correction terms. Technical details on the regularization scheme and on subleading terms in the $1 / \mathrm{m}$ expansion can be found in appendix B. We find:

$$
\begin{aligned}
J(p) & =J\left(-i z_{0}\right)+J_{R}\left(p, z_{0}\right), \quad V_{\pi, S}(p)=V_{\pi, R}(p)+\left(c-\frac{1}{2}\right) J(p), \\
J_{\pi, S}(p) & =J_{\pi, R}\left(p, z_{0}\right)+\bar{J}_{\pi}\left(-i z_{0}\right)+\left(c-\frac{1}{2}\right) J^{2}(p) .
\end{aligned}
$$


Note that here we also drop a contribution from the pion production cut in $J_{\pi, S}(p)$ (see (B.40)). The subtraction point $z_{0}$ will ultimately be identified with the pseudo-bound state pole position in the chiral limit. If $z_{0}>0$ the subtraction point lies on the second Riemann sheet of $p=\sqrt{s / 4-m^{2}}$. The renormalized bubble loop functions $J_{R}\left(p, z_{0}\right)$ and $J_{\pi, R}\left(p, z_{0}\right)$ vanish at $p=-i z_{0}$ by definition. The analytic expressions for the renormalized loop functions read:

$$
\begin{aligned}
J_{R}\left(p, z_{0}\right) & =\frac{m}{2 \pi}\left(-z_{0}+i p\right), \quad V_{\pi, R}(p)=\frac{m m_{\pi}}{8 \pi} \frac{m_{\pi}}{p} i \ln \left(1-i \frac{2 p}{m_{\pi}}\right), \\
J_{\pi, R}\left(p, z_{0}\right) & =-2 \frac{m^{2} m_{\pi}^{2}}{(4 \pi)^{2}} \ln \left(\frac{m_{\pi}-2 i p}{m_{\pi}-2 z_{0}}\right) .
\end{aligned}
$$

All divergencies sit in the one-loop and two-loop subtraction coefficients $J\left(-i z_{0}\right)$ and $\bar{J}_{\pi}\left(-i z_{0}\right)$ which may be estimated in terms of a cutoff parameter $\Lambda$ introduced in the s-channel dispersion relation (see (17)):

$$
J\left(-i z_{0}\right) \sim \frac{m}{\pi} \Lambda, \quad \bar{J}_{\pi}\left(-i z_{0}\right) \sim \frac{m^{2} m_{\pi}^{2}}{(4 \pi)^{2}} \ln \left(\frac{\Lambda^{2}}{m_{\pi}^{2}}\right)
$$

We point out that the vertex function $V_{\pi, S}(p)$ is rendered finite by the unique choice $c=1 / 2$ whereas the two-loop bubble $J_{\pi, S}(p)$ requires a further subtraction even at $c=1 / 2$. This reflects our renormalization procedure (41) and (42). The vertex function is renormalized by an appropriate 'counter loop' leading to the renormalization point independent result $V_{\pi, R}(p)$. The bubble function $J_{\pi, S}(p)$ is renormalized in two steps. The counter loops lead to a function $\bar{J}_{\pi}(p)$ which defines the renormalized loop modulo a subtraction $J_{\pi, R}\left(p, z_{0}\right)=\bar{J}_{\pi}(p)-\bar{J}_{\pi}\left(-i z_{0}\right)$. This leads to a subtraction point dependent result for $J_{\pi, R}\left(p, z_{0}\right)$. Note that here we are implicitly assuming that the onshell irreducible interaction kernel $\hat{K}_{\pi}$ is applied (see appendix B.3).

Consistency of our L-counting rule requires the subtraction constant $c$ to be rather close to one half. For example if $c$ deviates largely from $1 / 2$ the term proportional to $\left(c-\frac{1}{2}\right) J\left(-i z_{0}\right) J_{R}\left(p, z_{0}\right)$ in our expression for $J_{\pi, S}$ must not be counted as chiral order two since $g_{A}^{2} \lambda^{2} J\left(-i z_{0}\right)>1$. We count $(c-\bar{c}) \sim Q$ and therewith confirm $V_{\pi, S}(p) \sim Q$ and $J_{\pi, S}(p)-J_{\pi, S}\left(-i z_{0}\right) \sim Q^{2}$. Note that according to $(70) J_{\pi, S}(p)$ carries in fact the leading chiral power $Q$. This result generalizes to the $\mathrm{n}$-th order bubble $J_{\pi^{n}, S}(p)$. The anomalous contribution to all orders is readily anticipated

$$
J_{\pi^{n}, S}(p)=\left(c-\frac{1}{2}\right)^{n} J^{n+1}\left(-i z_{0}\right)+\mathcal{O}\left(Q^{n+1}\right)
$$

and leads to $J_{\pi^{n}, S}(p) \sim Q^{n}$. 
We proceed and express the not yet specified two-loop function $V_{\pi \pi, S}(p)$ and three-loop function $J_{\pi \pi, S}(p)$ of (65) in terms of the renormalized loops $V_{\pi \pi, R}(p)$ and $J_{\pi \pi, R}\left(p, z_{0}\right)$

$$
\begin{aligned}
V_{\pi \pi, S}(p) & =V_{\pi \pi, R}(p)+\left(c-\frac{1}{2}\right) J(p) V_{\pi, R}(p)+\left(c-\frac{1}{2}\right)^{2} J^{2}(p) \\
& +\left(c-\frac{1}{2}\right)\left(\bar{J}_{\pi}\left(-i z_{0}\right)+J_{\pi, R}\left(p, z_{0}\right)\right) \\
J_{\pi \pi, S}(p) & =J_{\pi \pi, R}\left(p, z_{0}\right)+\bar{J}_{\pi \pi}\left(-i z_{0}\right)+\left(c-\frac{1}{2}\right)^{2} J^{3}(p) \\
& +2\left(c-\frac{1}{2}\right) J(p)\left(\bar{J}_{\pi}\left(-i z_{0}\right)+J_{\pi, R}\left(p, z_{0}\right)\right)
\end{aligned}
$$

at leading order in the $1 / m$ expansion. We suppress here subleading contributions from pion production cuts. All divergencies in (74) sit in the previously introduced power divergent one-loop bubble subtraction $J\left(-i z_{0}\right)$ and the $\log$ divergent two-loop bubble subtraction $J_{\pi}\left(-i z_{0}\right)$. The three-loop subtraction constant $J_{\pi \pi}\left(-i z_{0}\right)$ is in fact finite:

$$
\bar{J}_{\pi \pi}\left(-i z_{0}\right)=\frac{m^{3} m_{\pi}^{3}}{(4 \pi)^{3}}\left(\ln (16)+\mathcal{O}\left(\frac{z_{0}}{m_{\pi}}\right)\right)
$$

The multiple powers of $J^{n}\left(-i z_{0}\right) \sim \Lambda^{n}$ in (74) reflect the presence of overlapping divergencies in the two-loop vertex and three-loop bubble functions. They are easily disentangled by proper loop subtractions and as shown in appendix B. Note that our result (74) confirms the anomalous part of $J_{\pi \pi, S}(p)$ as anticipated in (73). It is straightforward to derive analytic expressions for the renormalized loop functions. Again we find it most economic to deduce the analytic form of the loop functions from their imaginary parts by means of their known analytic structure. We drop $1 / m$ correction terms and present the leading renormalized loop functions

$$
\begin{aligned}
& V_{\pi \pi, R}(p)= \frac{m^{2} m_{\pi}^{2}}{(4 \pi)^{2}} \frac{m_{\pi}^{2}}{4 p^{2}}\left(\operatorname{Li}_{2}\left(\frac{1}{2}+\frac{i p}{m_{\pi}}\right)-2 \operatorname{Li}_{2}\left(\frac{1}{2} \frac{m_{\pi}}{m_{\pi}-i p}\right)\right. \\
&+\left.\left.\operatorname{Li}_{2}\left(\frac{1}{2}\right)-\ln ^{2}\left(\frac{m_{\pi}-2 i p}{2 m_{\pi}-2 i p}\right)-\ln \left(\frac{1}{2}-\frac{i p}{m_{\pi}}\right) \ln (2)\right)\right) \\
& \bar{J}_{\pi \pi}(p)=-i \frac{m^{3} m_{\pi}^{3}}{(4 \pi)^{3}} \frac{m_{\pi}}{p}\left(2 \operatorname{Li}_{2}\left(\frac{1}{2} \frac{m_{\pi}}{m_{\pi}-i p}\right)-\frac{i p}{m_{\pi}} \ln (16)\right. \\
&\left.\left.+\ln ^{2}\left(\frac{m_{\pi}-2 i p}{2 m_{\pi}-2 i p}\right)-\frac{\pi^{2}}{6}\right)\right)+\bar{J}_{\pi \pi}(0)
\end{aligned}
$$


with $J_{\pi \pi, R}(p, z)=\bar{J}_{\pi \pi}(p)-\bar{J}_{\pi \pi}(-i z)^{7}$. Again we confirm the anticipated chiral powers of the loop functions with $V_{\pi \pi, S}(p) \sim Q^{2}$ and $J_{\pi \pi, S}(p)-J_{\pi \pi, S}\left(-i z_{0}\right) \sim$ $Q^{3}$. The analytic results (76) explicitly demonstrate the presence of a branch point at $p=-i m_{\pi}$ in the two-loop vertex function $V_{\pi \pi, R}(p)$ and three-loop bubble function $J_{\pi \pi, R}\left(p, z_{0}\right)$. Note that the polylog function $\operatorname{Li}_{2}(x)$ has one branch point at $x=1$. It remains the contribution from the pion-nucleon box diagram to the s-wave scattering amplitude. The relevant combination

$$
B_{1}(u, t)-\frac{u-t}{4 m^{2}} B_{4}(u, t)-\frac{u+t}{4 m^{2}} B_{5}(u, t)=B(u, t)-V_{\pi, R}(p)+\frac{1}{4} J(p)(
$$

is conveniently expressed in terms of the reduced pion-nucleon box function $B(u, t)$, the renormalized vertex loop $V_{\pi, R}(p)$ and $J(p)$. We derive the analytic expression

$$
\begin{aligned}
B(u, t) & =\frac{1}{4 \pi} \frac{m m_{\pi}^{4}}{\sqrt{-t b(u, t)}}\left(i \operatorname{arctanh}\left(\sqrt{\frac{t(u+t)}{b(u, t)}}\right)\right. \\
& +\frac{1}{2} \arctan \left(\frac{\sqrt{-t b(u, t)}}{4 m_{\pi}^{3}}-\frac{\sqrt{-u-t}}{m_{\pi}}\left(1-\frac{t}{4 m_{\pi}^{2}}\right)\right) \\
& \left.+\frac{1}{2} \arctan \left(\frac{\sqrt{-t b(u, t)}}{4 m_{\pi}^{3}}+\frac{\sqrt{-u-t}}{m_{\pi}}\left(1-\frac{t}{4 m_{\pi}^{2}}\right)\right)\right) \\
b(u, t) & =4 m_{\pi}^{4}-4 m_{\pi}^{2}(u+t)+t(u+t) .
\end{aligned}
$$

The result (78) confirms the expected chiral order of the reduced diagram with $B(u, t) \sim Q$.

Finally we collect all terms introduced in (65) and (67) relevant for s-wave scattering according to (58)

$$
\begin{aligned}
T_{\left[{ }^{1} S_{0}\right]}(u, t) & =2 g_{A}^{2} \lambda^{2}\left(c-\frac{1}{2}\right)\left(1+\left(c-\frac{1}{2}\right) J(p)\right)+4 g_{A}^{4} \lambda^{4}\left(c-\frac{1}{2}\right) V_{\pi, R}(p) \\
& -\frac{1}{2} g_{A}^{2} \lambda^{2}\left(\frac{m_{\pi}^{2}}{u-m_{\pi}^{2}}+\frac{m_{\pi}^{2}}{t-m_{\pi}^{2}}\right)+g_{A}^{4} \lambda^{4}(B(u, t)+B(t, u)) \\
& +2 \frac{\left(1+g_{A}^{2} \lambda^{2} V_{\pi, S}(p)\right)^{2}+g_{A}^{2} \lambda^{2} V_{\pi \pi, S}(p)}{g_{\sigma, S}^{-1}(s)-J(p)-g_{A}^{2} \lambda^{2} J_{\pi, S}(p)-g_{A}^{4} \lambda^{4} J_{\pi \pi, S}(p)}
\end{aligned}
$$

$\overline{7 \text { Here }}$ we recall the definition of the polylog function

$$
\mathrm{Li}_{2}(x)=\int_{x}^{0} d t \frac{\ln (1-t)}{t}=\sum_{k=1}^{\infty} \frac{x^{k}}{k^{2}}
$$


at chiral order $Q$. According to the discussion in section 2 we refrain from expanding the denominator in (79). Expression (79) serves as a convenient starting point for any further expansion implied by scenario I and II of section 2 .

It is worth pointing out a generic property of the renormalized loop functions $V_{\pi^{n}, R}(p)$ and $J_{\pi^{n}, R}\left(p, z_{0}\right)$. Since they satisfy a dispersion relation in the Mandelstam variable $s$ they are in fact analytic in $p$ except for multiple branch points at $p=-i k m_{\pi} / 2$ with $0 \geq n \geq k$. This convenient property can be applied to derive the analytic form of the loop function in terms of its imaginary part (see appendix B). The s-wave scattering amplitude $T_{\left[{ }^{1} S_{0}\right]}(p)$ shows also branch points on the upper complex half plane with $p=+i k m_{\pi} / 2$. These branch points are, however, exclusively induced by the s-wave projected multiple pion exchanges of $T_{\pi, S}$. We perform the angle average of the one-pion exchange contribution and $B(u, t)$ according to (57)

$$
\begin{aligned}
R_{\pi}(p)=-\frac{1}{4} \int \frac{d \Omega}{4 \pi}\left(\frac{m_{\pi}^{2}}{u-m_{\pi}^{2}}+\frac{m_{\pi}^{2}}{t-m_{\pi}^{2}}\right)=\frac{m_{\pi}^{2}}{8 p^{2}} \ln \left(1+\frac{4 p^{2}}{m_{\pi}^{2}}\right) \\
R_{\pi \pi}(p)=\int \frac{d \Omega}{4 \pi} B(u, t) \\
=\frac{m m_{\pi}}{8 \pi} \frac{m_{\pi}^{3}}{8 p^{3}}\left(i \ln ^{2}\left(\frac{1}{2}+\frac{i p}{m_{\pi}}\right)-2 i \operatorname{Li}_{2}\left(\frac{1}{2}+\frac{i p}{m_{\pi}}\right)\right. \\
-2 i \operatorname{Li}_{2}\left(\frac{1}{2} \frac{m_{\pi}}{m_{\pi}+i p}\right)+2 i \operatorname{Li}_{2}\left(\frac{1}{2} \frac{m_{\pi}}{m_{\pi}-i p}\right) \\
+2 \ln \left(\frac{m_{\pi}^{2}+4 p^{2}}{4 m_{\pi}^{2}+4 p^{2}}\right)\left(\arctan \left(\frac{2 p}{m_{\pi}}\right)-\arctan \left(\frac{p}{m_{\pi}}\right)\right) \\
\left.+2 i \ln (2) \ln \left(\frac{1}{4}+\frac{p^{2}}{m_{\pi}^{2}}\right)+2 i \ln ^{2}(2)+i \frac{\pi^{2}}{6}\right)
\end{aligned}
$$

The expressions (80) confirm that indeed the function $R_{\pi}(p)$ shows branch points at $p= \pm i m_{\pi} / 2$ and $R_{\pi \pi}(p)$ branch points at $p= \pm i m_{\pi}$ and $p=$ $\pm i m_{\pi} / 2$ as expected.

We conclude that the chiral L-counting rule derived by means of the s-channel dispersion relation in section 2 is confirmed explicitly for the loop functions considered here. Let us shed some light on the convergence property of the chiral expansion. A convenient measure is the ratio of the one-to-two loop and two-to-three loop subtraction constants:

$$
g_{A}^{2} \lambda^{2} \frac{J_{\pi, S}(0)}{J(0)} \simeq g_{A}^{2} \lambda^{2} \frac{m m_{\pi}}{4 \pi} \frac{m_{\pi}}{\Lambda} \ln \left(\frac{\Lambda^{2}}{m_{\pi}^{2}}\right)
$$




$$
g_{A}^{2} \lambda^{2} \frac{J_{\pi \pi, S}(0)}{J_{\pi, S}(0)} \simeq g_{A}^{2} \lambda^{2} \frac{m m_{\pi}}{4 \pi} \frac{\ln (4)}{\ln \left(\frac{\Lambda}{m_{\pi}}\right)}
$$

at $c=\bar{c}$. The typical cutoff $\Lambda \simeq 600 \mathrm{MeV}$ implies reasonable small ratios of about 0.27 and 0.35 for the leading and subleading ratio respectively. Finally consider also the ratio of leading to subleading vertex functions:

$$
g_{A}^{2} \lambda^{2} \frac{V_{\pi \pi, R}(0)}{V_{\pi, R}(0)}=g_{A}^{2} \lambda^{2} \frac{m m_{\pi}}{4 \pi} \ln (2) \simeq 0.28
$$

evaluated at $p^{2}=0$. All together we find good convergence behavior of the chiral expansion. The underlying expansion parameter appears to be roughly $1 / 3$. Before presenting further details of our chiral expansion scheme we compare the analytic results with a calculation by Fogel[29] obtained in the static pion limit.

\subsection{Static pion limit}

It is instructive to compare our result (65) with a corresponding calculation performed with static pion propagators. As an example we consider here the one-pion exchange contributions $R_{\pi}(p)$ and $R_{\pi \pi}(p)$ (see (80)). The limit of static pions is accessed most economically by considering the Schrödinger equation of a Yukawa potential

$$
\psi^{\prime \prime}(r)+\left(p^{2}+g_{\pi} m_{\pi} \frac{e^{-m_{\pi} r}}{r}\right) \psi(r)=0
$$

with the coupling constant $g_{\pi}=g_{A}^{2} m_{\pi} m /\left(16 \pi f_{\pi}^{2}\right) \simeq 0.45$. This problem was solved analytically to lowest orders in the coupling constant by Fogel [29]. The s-wave scattering phase shift $\delta_{\left[{ }^{1} S_{0}\right]}(p)$

$$
\tan \delta_{\left[{ }^{1} S_{0}\right]}(p)=\frac{\Im f(p)}{\Re f(p)}
$$

is expressed in terms of the Jost function, $f(p)$, which is the solution of (83) with asymptotic behavior $\sim e^{-i p r}$ evaluated at $r=0$. In [29] the Jost function is evaluated perturbatively

$$
f(p)=1+\sum_{n=1}^{\infty} g_{\pi}^{n} f_{n}(p)
$$

with analytic results given for leading and subleading terms: 


$$
\begin{aligned}
f_{1}(p)=\frac{i m_{\pi}}{2 p} \ln \left(1+2 \frac{i p}{m_{\pi}}\right) & \\
f_{2}(p)=-\frac{m_{\pi}^{2}}{4 p^{2}} & \left(\frac{1}{2} \ln ^{2}\left(1+2 \frac{i p}{m_{\pi}}\right)+\frac{\pi^{2}}{12}-\operatorname{Li}_{2}\left(\frac{2 i p-m_{\pi}}{2 i p+m_{\pi}}\right)\right. \\
& \left.+2 \operatorname{Li}_{2}\left(\frac{-m_{\pi}}{2 i p+m_{\pi}}\right)\right) .
\end{aligned}
$$

It is found $[29,30]$ that for physical coupling $g_{\pi} \simeq 0.45$ the perturbative expansion of the Jost function is rapidly converging for momenta $2 p>g_{\pi} m_{\pi} \simeq 70$ $\mathrm{MeV}$. The convergence property is in fact improving significantly as $p$ gets larger. The next moment $f_{3}(p)$ was evaluated by Friman [30].

We confront our result with the analytic expressions of Fogel by matching the s-wave scattering amplitude, $T_{\left[{ }^{1} S_{0}\right]}(p)$, at leading and subleading order in the coupling constant $g_{\pi}$ :

$$
T_{\left[{ }^{1} S_{0}\right]}(p)=\frac{8 \pi}{\sqrt{s} p} \frac{\Im f(p)}{\Re f(p)-i \Im f(p)}
$$

We identify

$$
\begin{aligned}
R_{\pi}(p) & \leftrightarrow \frac{m_{\pi}}{2 p} \Im f_{1}(p) \\
R_{\pi \pi}(p) & \leftrightarrow \frac{m m_{\pi}^{2}}{8 \pi p}\left(\Im f_{2}(p)-\Im f_{1}(p)\left(\Re f_{1}(p)-i \Im f_{1}(p)\right)\right)
\end{aligned}
$$

and conclude that, as to be expected, the terms agree identically. For a discussion and evaluation of $1 / m$ correction terms as they evolve from relativistic Feynman diagrams see appendix B.1.

\subsection{Expansion of effective coupling function}

In this section we will further prepare the ground to generalize (25) for the case in which the pion dynamics is included explicitly in the theory. Starting from (79) we will specify how to identify and expand the renormalized coupling function.

The scattering amplitude (79) involves the subtracted coupling function $g_{\sigma, S}=$ $g-g_{A}^{2} \lambda^{2} c$. Within our renormalization procedure (41) and (42), which holds for both scenarios I) and II), the loop subtraction coefficient $\bar{c}$ is determined by enforcing finite vertex functions $V_{\pi^{n}, S}(p)$. At leading orders this is achieved with $\bar{c}=1 / 2+\mathcal{O}\left(Q^{2}\right)$ corresponding to the 'local' part of the one-pion exchange potential (see (79)). The remaining divergencies sit in the bubble loop 
functions $J_{\pi^{n}, S}(s)$ (see $(70,74)$ ) and can be absorbed into the bare coupling function $g\left(p, m_{\pi}\right)$ by a subtraction procedure. To this end it is convenient to introduce the auxiliary coupling function $g_{H}\left(s, m_{\pi} ; c\right)$, the basic building block in (79),

$$
\begin{aligned}
g_{H}^{-1}\left(s, m_{\pi} ; c\right) & =g_{\sigma, S}^{-1}\left(s, m_{\pi} ; c\right)-J\left(-i z_{0}\right) \\
& -g_{A}^{2} \lambda^{2} J_{\pi, S}\left(-i z_{0}\right)-g_{A}^{4} \lambda^{4} J_{\pi \pi, S}\left(-i z_{0}\right)+\cdots, \\
g_{R}\left(p, m_{\pi}\right) & =g_{H}\left(s, m_{\pi} ; \bar{c}\right)
\end{aligned}
$$

which then defines the renormalized coupling function if evaluated at $c=\bar{c}$. In (89) we consider the bubble loops $J_{\pi^{n}, S}(p)$ as a function of the momentum $p$. The auxiliary coupling function $g_{H}\left(s, m_{\pi} ; c\right)$ depends on the subtraction $c$ via the coupling function $g_{\sigma, S}$ and via the explicit dependence of the bubble loop functions $J_{\pi^{n}, S}(p)$. The subtraction point $z_{0}=z\left(m_{\pi}=0\right)$ is identified with the pseudo-bound state pole position in the chiral limit.

The bare coupling function $g\left(s, m_{\pi}^{2}\right)$ in $g_{H}\left(s, m_{\pi} ; c\right)$ permits a Taylor expansion in $p^{2}$ and $m_{\pi}^{2}$. In scenario I) the bare coupling function $g\left(s, m_{\pi}^{2}\right)$ and the renormalized coupling function $g_{R}\left(p, m_{\pi}\right)$ are expanded as follows:

$$
\begin{aligned}
g\left(s, m_{\pi}^{2}\right) & =\lambda^{2} \sum_{n, k} \beta_{2 n}^{(2 k)}\left(p^{2}+z_{0}^{2}\right)^{n} m_{\pi}^{2 k}, \\
g_{R, I}^{-1}\left(p, m_{\pi}\right) & =\frac{m}{2 \pi} \sum_{n, k} \zeta_{2 n}^{(k)}\left(p^{2}+z_{0}^{2}\right)^{n} m_{\pi}^{k} .
\end{aligned}
$$

Note that in (90) we slightly reorganized the expansion of the bare coupling function as compared to (15). The function $g\left(s, m_{\pi}\right)$ represents an infinite hierarchy of interaction terms of the chiral Lagrangian with increasing number of derivative couplings and increasing degree of explicit chiral symmetry breaking. The naturalness assumption leads to $\beta_{n}^{(m)} \sim \Lambda_{n a t}^{-n-m}$ and $\Lambda_{\text {nat. }}=\Lambda_{\text {nat. }}^{(I)} \simeq m_{\rho} / 2$ at given cutoff $\Lambda \simeq \Lambda_{\text {nat. }}$.

The renormalized coupling constants $\zeta_{2 n}^{(k)}$ can be expressed in terms of the bare parameters $\beta_{2 n}^{(2 k)}$ and the subtraction coefficients $J_{\pi^{n}, S}\left(-i z_{0}\right)$. We expand the inverse auxiliary coupling function $g_{H}^{-1}\left(s, m_{\pi} ; c\right)$ around the point $c=\bar{c}$ (see (48)). The leading terms read:

$$
\begin{aligned}
\frac{\lambda^{2}}{g\left(s, m_{\pi}\right)-c g_{A}^{2} \lambda^{2}} & =\frac{1}{\beta_{0}^{(0)}-g_{A}^{2} / 2}-\frac{\beta_{0}^{(2)} m_{\pi}^{2}+\beta_{2}^{(0)}\left(p^{2}+z_{0}^{2}\right)}{\left(\beta_{0}^{(0)}-g_{A}^{2} / 2\right)^{2}} \\
& +\frac{g_{A}^{2}(c-\bar{c})}{\left(\beta_{0}^{(0)}-g_{A}^{2} / 2\right)^{2}}+\frac{g_{A}^{4}(c-\bar{c})^{2}}{\left(\beta_{0}^{(0)}-g_{A}^{2} / 2\right)^{3}}
\end{aligned}
$$




$$
-2 g_{A}^{2}(c-\bar{c}) \frac{\beta_{0}^{(2)} m_{\pi}^{2}+\beta_{2}^{(0)}\left(p^{2}+z_{0}^{2}\right)}{\left(\beta_{0}^{(0)}-g_{A}^{2} / 2\right)^{3}}+\mathcal{O}\left(Q^{3}\right) .
$$

where we count $c-\bar{c} \sim Q$. The divergent subtraction coefficients can now be absorbed into the bare parameters $\beta_{2 n}^{(2 k)}$. The linear divergence in $J\left(-i z_{0}\right)$ is absorbed into the bare coupling $\beta_{0}^{(0)}$ and the log-divergence in $\bar{J}_{\pi}\left(-i z_{0}\right)$ is absorbed into $\beta_{2}^{(0)}$ (see (72)). The leading order renormalized coupling constants $\zeta_{0}^{(2)}, \zeta_{2}^{(0)}$ and $\zeta_{0}^{(3)}$ can now be identified

$$
\begin{aligned}
\lambda^{2} J\left(-i z_{0}\right) & =\frac{1}{\left(\beta_{0}^{(0)}-g_{A}^{2} / 2\right)}, \quad \zeta_{2}^{(0)}=-\frac{2 \pi \beta_{2}^{(0)} / \lambda^{2}}{m\left(\beta_{0}^{(0)}-g_{A}^{2} / 2\right)^{2}}, \\
\zeta_{0}^{(2)} & =-g_{A}^{2} \lambda^{2} \frac{2 \pi}{m} \frac{\bar{J}_{\pi}\left(-i z_{0}\right)}{m_{\pi}^{2}}-\frac{2 \pi \beta_{0}^{(2)} / \lambda^{2}}{m\left(\beta_{0}^{(0)}-g_{A}^{2} / 2\right)^{2}}, \\
\zeta_{0}^{(3)} & =-g_{A}^{2} \lambda^{2} \frac{2 \pi}{m} \frac{\bar{J}_{\pi \pi}\left(-i z_{0}\right)}{m_{\pi}^{3}} .
\end{aligned}
$$

Note that the renormalized coupling function $g_{R}\left(p, m_{\pi}\right)$ includes non-analytic terms in the squared pion mass (e.g. $m_{\pi}^{3} \zeta_{0}^{(3)}$ ) from the bubble loop subtraction coefficients $J_{\pi^{n}, S}\left(-i z_{0}\right)$ not present in the bare coupling function $g\left(s, m_{\pi}^{2}\right)$. Moreover we point out that the inverse auxiliary coupling function $g_{H}^{-1}\left(s, m_{\pi} ; c\right)$ and the inverse renormalized coupling function $g_{R}^{-1}\left(p, m_{\pi}\right)$ agree up to chiral order $Q^{2}$ :

$$
g_{H}^{-1}\left(s, m_{\pi} ; c\right)=\zeta_{2}^{(0)} \frac{m}{2 \pi}\left(p^{2}+z_{0}^{2}\right)+\frac{m}{2 \pi} \zeta_{0}^{(2)} m_{\pi}^{2}+\mathcal{O}\left(Q^{3}\right) .
$$

This follows since (92) implies that the power divergent terms proportional to $(c-\bar{c})^{n}$ with $n=1,2$ in (91) are canceled by matching contributions from $J_{\pi, S}\left(-i z_{0}\right)$ and $J_{\pi \pi, S}\left(-i z_{0}\right)$ (see $(70,73)$ ). For the generalization of (93), which does show an explicit dependence on $c-\bar{c}$, valid up to chiral order $Q^{3}$ see (C.5).

We turn to scenario II). Most economically it may be considered as a particular resummation of scenario I) with:

$$
\begin{aligned}
g_{R, I I}^{-1}\left(p, m_{\pi}\right)=\frac{m}{2 \pi}\left(\xi_{\sigma} \frac{p^{2}+z_{0}^{2}}{p^{2}-\mu_{\sigma}^{2}}+\xi_{2}^{(0)}\left(p^{2}+z_{0}^{2}\right)\right. \\
\left.+\xi_{0}^{(2)} m_{\pi}^{2}+\xi_{0}^{(3)} m_{\pi}^{3}\right)+\mathcal{O}\left(Q^{4}\right) \\
\sum_{k=0}^{\infty} \zeta_{2 n}^{(k)} m_{\pi}^{k}=-\xi_{\sigma}\left(\mu_{\sigma}^{2}+z_{0}^{2}\right)^{-n}+\sum_{k=0}^{\infty} \xi_{2 n}^{(k)} m_{\pi}^{k}
\end{aligned}
$$


where $\mu_{\sigma}=\mu_{\sigma}\left(m_{\pi}\right)$ and $\xi_{\sigma}=\xi_{\sigma}\left(m_{\pi}\right)$. The bare coupling function $g\left(s, m_{\pi}^{2}\right)$ and the renormalized inverse coupling functions $g_{R, I I}^{-1}\left(p, m_{\pi}\right)$ of scenario II) are expanded around $p^{2}=\mu_{\sigma}^{2}$. At given order $\left(p^{2}-\mu_{\sigma}^{2}\right)^{n}$ the terms can then always be regrouped according to (94). Note that the form of the renormalized coupling functions in $(90,94)$ avoids a fine tuning of parameters which in (25) is required for the formation of the pseudo-bound state pole. The parameter $\mu_{\sigma}$ of scenario II) carries physical significance since it will describe the empirical fact that s-wave scattering at relative momentum $p \simeq 350 \mathrm{MeV}$ is interaction free. This phenomena reflects the well established subtle cancellation of attractive $\sigma$-exchange and repulsive $\omega$-exchange in phenomenological nucleon-nucleon potentials [24,25]. The expression (94) clearly demonstrate that as the value of $\mu_{\sigma}$ decreases the effective chiral power of the first term in $g_{R, I I}^{-1}$ changes dramatically: for $\mu_{\sigma} \gg \Lambda_{\text {nat. }}$ we find $Q^{2}$ whereas for $\mu_{\sigma} \ll \Lambda_{\text {nat }}$. we are led to $Q^{0}$. Note however that at momenta $p^{2} \ll \mu_{\sigma}^{2}$ the 'leading' term is suppressed by the factor $z_{0}^{2} / \mu_{\sigma}^{2}$ and therefore the 'subleading' term, in particular the $\xi_{0}^{(2)}$-term, becomes equally important. Recall that we expect $\mu_{\sigma} \sim 350$ $\mathrm{MeV}$ and $z_{0} \sim z \sim 8 \mathrm{MeV}$. Thus an expansion in ratios of 'subleading' over 'leading' term is converging for momenta $p^{2} \sim \mu_{\sigma}^{2}$ but turns useless for momenta $p^{2} \ll \mu_{\sigma}^{2}$. Our expansion for $g_{R, I I}^{-1}$, in contrast, is expected to converge well for momenta $\mu_{\sigma}^{2}-\Lambda_{\text {nat. }}^{(I I)}<p^{2}<\mu_{\sigma}^{2}+\Lambda_{\text {nat. }}^{(I I)}$ with $\Lambda_{\text {nat. }}^{(I I)} \simeq 600 \mathrm{MeV}$ (see section 2.2). This is further support for our claim that $T_{\sigma, S}$ must not be expanded in powers of $T_{\pi, S}($ see $(38))$.

\subsection{Inefficient chiral expansion (CE) scheme}

In this section we develop the scenario I) in the presence of perturbative pion dynamics. The denominator of (67) is systematically expanded in chiral powers. We call such an approach 'CE' scheme for future reference. The motivation of this section is twofold. On the one hand we show the failure of the CE scheme on the other hand we present our renormalization scheme at work. We will systematically express the scattering amplitude at subleading orders in terms of the physical parameters $z_{0}, \zeta_{k}^{(m)}$ introduced in the previous section (see $(97,92)$ ) rather than in terms of the cutoff and bare coupling parameters. Therewith manifest scheme independent results are obtained. In particular we will demonstrate the independence of the scattering amplitude on the subtraction function $\mathrm{c}(\mathrm{s})$ in a perturbative sense: if the scattering amplitude is expanded up to chiral order $Q^{n}$ with $c-\bar{c} \sim Q$ any residual c-dependence contributes at order $Q^{n+1}$. The perturbative cancellation of any $c$-dependence demonstrates that physical results do not depend on how to distribute strength among $K_{\pi, S}$ and $K_{\sigma, S}$ in $(36,37)$. For the full solution of the Bethe-Salpeter equation this is of course true by construction. For the truncated scattering amplitude this is, however, a non-trivial constraint. 
According to (93) the auxiliary coupling function $g_{H}^{-1}\left(s, m_{\pi} ; c\right)$ carries leading chiral order two. This leads to an s-wave scattering amplitude of leading chiral order minus one:

$$
T_{\left[{ }^{1} S_{0}\right]}(p)=-\frac{2}{J_{R}\left(p, z_{0}\right)}+\mathcal{O}\left(Q^{0}\right)=\frac{4 \pi}{m} \frac{i}{p+i z_{0}}+\mathcal{O}\left(Q^{0}\right)
$$

The result (95) is certainly not a surprise. One recovers the effective range theory with $r=0$. The pole in (95) at $p=-i z_{0}$ is the regularization scheme dependent result of fine-tuned bare coupling strength and intrinsic cutoff parameter. So far the chiral dynamics shows no quantitative predictive power. First non-trivial consequence are expected at subleading order. Note that the leading order result does not yet reveal the necessity to expand around $c=\bar{c}$ with $\bar{c}=1 / 2+\mathcal{O}\left(Q^{2}\right)$. This can first be seen at subleading order.

We proceed and turn to the zeroth order amplitude. At this order the auxiliary coupling function $g_{H}\left(s, m_{\pi} ; c\right)$ can be identified with the renormalized coupling function $g_{R}\left(p, m_{\pi}\right)$ (see $\left.(93)\right)$. We collect all terms of chiral order zero:

$$
\begin{aligned}
T_{\left[{ }^{1} S_{0}\right]}(p) & =-\frac{2}{J_{R}(p, z)}+2 g_{A}^{2} \lambda^{2} R_{\pi}(p)-2 \frac{g_{R}^{-1}\left(p, m_{\pi}\right)}{J_{R}^{2}\left(p, z_{0}\right)} \\
& +g_{A}^{2} \lambda^{2}\left(1-4 \frac{V_{\pi, R}(p)}{J_{R}\left(p, z_{0}\right)}+2 \frac{J_{\pi, R}\left(p, z_{0}\right)}{J_{R}^{2}\left(p, z_{0}\right)}\right)+\mathcal{O}(Q) .
\end{aligned}
$$

The result (96) is expressed in terms of the physical parameters in $g_{R}\left(p, m_{\pi}\right)$ and a set of renormalized loop functions given in $(71,80)$. We point out that in (96) the explicit c-dependence induced by the loop functions $V_{\pi, S}(p)$ and $J_{\pi, S}(p)$ (see $(70)$ ) cancels identically upon expansion. Therefore the zeroth order scattering amplitude (96) is manifestly independent of the subtraction procedure. From the analysis of the effective range theory we expect the scattering amplitude to exhibit a single pole at $p=-i z$ rather than at $p=-i z_{0}$. We conjecture an expansion of the pseudo-bound state pole position $z\left(m_{\pi}\right)$ in powers of the pion mass:

$$
z\left(m_{\pi}\right)=z_{0}+\sum_{m=2}^{\infty} \bar{\zeta}_{m} m_{\pi}^{m}
$$

with $\bar{\zeta}_{m} \sim \Lambda_{\text {nat. }}^{-m+1}$ and $z_{0} \sim m_{\pi} \sim Q$. Therefore an expansion scheme which preserves the proper low energy structure of the scattering amplitude (27) requires a particular summation. In order to comply with (27) we expand all bare poles in terms of powers of the physical pole term and then impose the cancellation of all multiple poles at $p=-i z$. The expansion of the leading order pole reads: 


$$
\begin{aligned}
-\frac{2}{J_{R}\left(p, z_{0}\right)} & =-\frac{2}{J_{R}(p, z)}+2 \bar{\zeta}_{2} \frac{m}{2 \pi} \frac{m_{\pi}^{2}}{J_{R}^{2}(p, z)}-\left(\bar{\zeta}_{2}\right)^{2} \frac{m^{2}}{2 \pi^{2}} \frac{m_{\pi}^{4}}{J_{R}^{3}(p, z)} \\
& +2 \bar{\zeta}_{3} \frac{m}{2 \pi} \frac{m_{\pi}^{3}}{J_{R}^{2}(p, z)}+\cdots
\end{aligned}
$$

where we apply our notation (97). At subleading order this leads to $\bar{\zeta}_{2}=\zeta_{0}^{(2)}$.

We now turn to the comparison of our result (96) with the effective range theory. It is instructive to make the analytic structure of our result more explicit. We rewrite (96) and present the central result of this section in terms of the reduced functions $R_{V, \pi}^{(1)}$ and $R_{J, \pi}^{(2)}$ and the one-pion exchange contribution $R_{\pi}(p)$ (see $\left.(80)\right)$ :

$$
\begin{aligned}
T_{\left[{ }^{1} S_{0}\right]}(p) & =\frac{4 \pi}{m} \frac{i w}{p+i z}+\frac{4 \pi}{m} \zeta_{2}^{(0)} \\
& +2 g_{A}^{2} \lambda^{2}\left(R_{\pi}(p)-2 R_{V, \pi}^{(1)}\left(p, z_{0}\right)+R_{J, \pi}^{(2)}\left(p, z_{0}\right)\right)+\mathcal{O}(Q) .
\end{aligned}
$$

Here we introduced a convenient notation: for any given loop function $F(p, z)$ we define the n-th order residual function, $R_{F}^{(n)}(p, z)$, by:

$$
R_{F}^{(n)}(p, z)=J_{R}^{-n}(p, z)\left(F(p, z)-\sum_{k=0}^{n-1} \frac{1}{k !}(p+i z)^{k} \partial_{p}^{k} F(-i z, z)\right)
$$

Note that for a given loop function $F(p, z)$ which is regular at $p=-i z$ also the set of residual functions $R_{F}^{(n)}(p, z)$ is regular at $p=-i z$ by construction. The pole residuum $w$ is:

$$
\begin{aligned}
w & =1+g_{A}^{2} \lambda^{2}\left(2 V_{\pi, R}\left(-i z_{0}\right)-\frac{2 \pi}{m} \frac{\partial}{\partial p} J_{\pi, R}\left(-i z_{0}, z_{0}\right)\right) \\
& -2 \zeta_{2}^{(0)} z_{0}+\mathcal{O}\left(Q^{2}\right) .
\end{aligned}
$$

With (99) we arrive at a representation of the scattering amplitude anticipated in the previous section when discussing the low energy limit. The amplitude is the sum of the pseudo-bound state pole and a smooth non-polynomial remainder, which takes care of the non-trivial pion dynamics.

In Fig. 1) we show the result of a low energy fit to the empirical scattering amplitude. For momenta $p<50 \mathrm{MeV}$ the scattering amplitude is well reproduced. It is also shown that the unitarity condition is violated strongly at momenta $p<150 \mathrm{MeV}$. This is a disappointing result since already the effective range approximation works quite accurately up to momenta $p \simeq 150$ $\mathrm{MeV}$. We conclude that it would be misleading to confront the CE result (99) with the empirical scattering phase shift. 


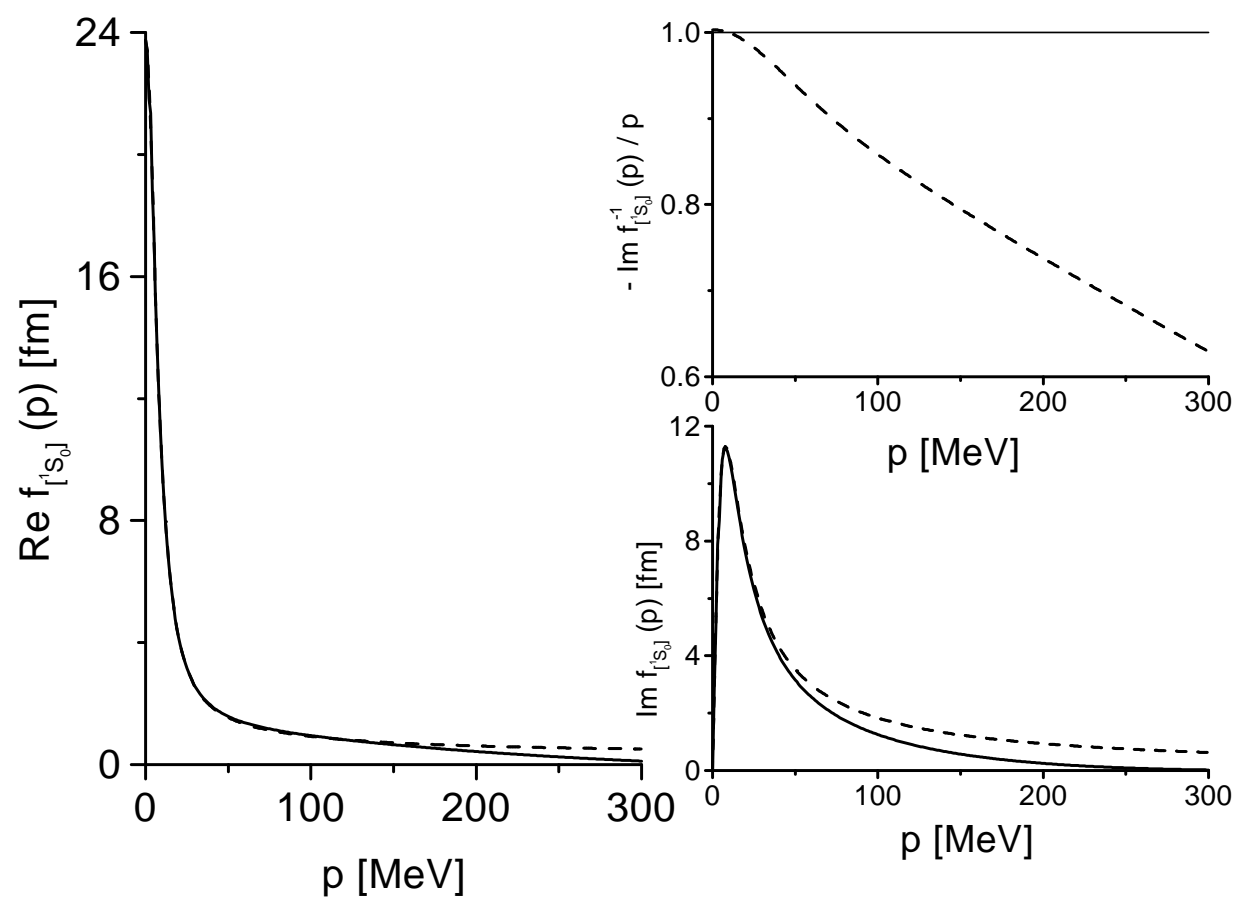

Fig. 1. The left hand figure shows the real part of the np-scattering amplitude $f_{\left[{ }^{1} S_{0}\right]}(p)=\sqrt{s} T_{\left[{ }^{1} S_{0}\right]}(p) /(8 \pi)$. The right hand figure shows the imaginary part of the np-scattering amplitude $\Im f_{\left[{ }^{1} S_{0}\right]}(p)$ and the unitarity function $-\Im f_{\left[{ }^{1} S_{0}\right]}^{-1}(p) / p$, which is one if unitarity holds. The solid lines are from the PWA93 solution of [4] and the dashed lines are the result of the zeroth order CE scheme with $\zeta_{2}^{(0)}=0.380$ fm, $z_{0}=8.35 \mathrm{MeV}$ and $z=7.89 \mathrm{MeV}$ in (99).

It is instructive to analyze our result in more detail and apply a further numerical expansion to the pole residuum $w$ and the effective scattering length $b_{\mathrm{eff}}^{(0)}$ :

$$
\begin{aligned}
1-w & =\frac{m m_{\pi}}{4 \pi} g_{A}^{2} \lambda^{2}\left(2 \frac{z_{0}}{m_{\pi}}+\frac{16}{3} \frac{z_{0}^{2}}{m_{\pi}^{2}}+\mathcal{O}\left(\frac{z_{0}^{3}}{m_{\pi}^{3}}\right)\right)+2 \zeta_{2}^{(0)} z_{0}+\mathcal{O}\left(Q^{2}\right) \\
b_{\mathrm{eff}}^{(0)} & =-\zeta_{2}^{(0)}-\frac{m}{4 \pi} g_{A}^{2} \lambda^{2}\left(1+\frac{8}{3} \frac{z_{0}}{m_{\pi}}+\frac{8 z_{0}^{2}}{m_{\pi}^{2}}+\mathcal{O}\left(\frac{z_{0}^{3}}{m_{\pi}^{3}}\right)\right)+\mathcal{O}(Q)
\end{aligned}
$$

The free parameter $\zeta_{2}^{(0)}$ and $z_{0}$ are now adjusted to reproduce the empirical residuum $w \simeq 0.90$ and the effective scattering length $b_{\mathrm{eff}}^{(0)} \simeq-1.15 \mathrm{fm}$ (see (29)). We obtain the values $\zeta_{2}^{(0)} \simeq 0.38 \mathrm{fm}$ and $z_{0} \simeq 8.35 \mathrm{MeV}$. The scheme dependent bare parameters $\beta_{0}^{(0)}, \beta_{2}^{(0)}$ and $\beta_{0}^{(2)}$ follow with (92). The remaining physical parameter $\zeta_{0}^{(2)}$ follows with $z=z_{0}+\zeta_{0}^{(2)} m_{\pi}^{2}+\cdots$ which leads to $\zeta_{0}^{(2)} \simeq-0.0047 \mathrm{fm}$. It is important to inspect also the next moment 


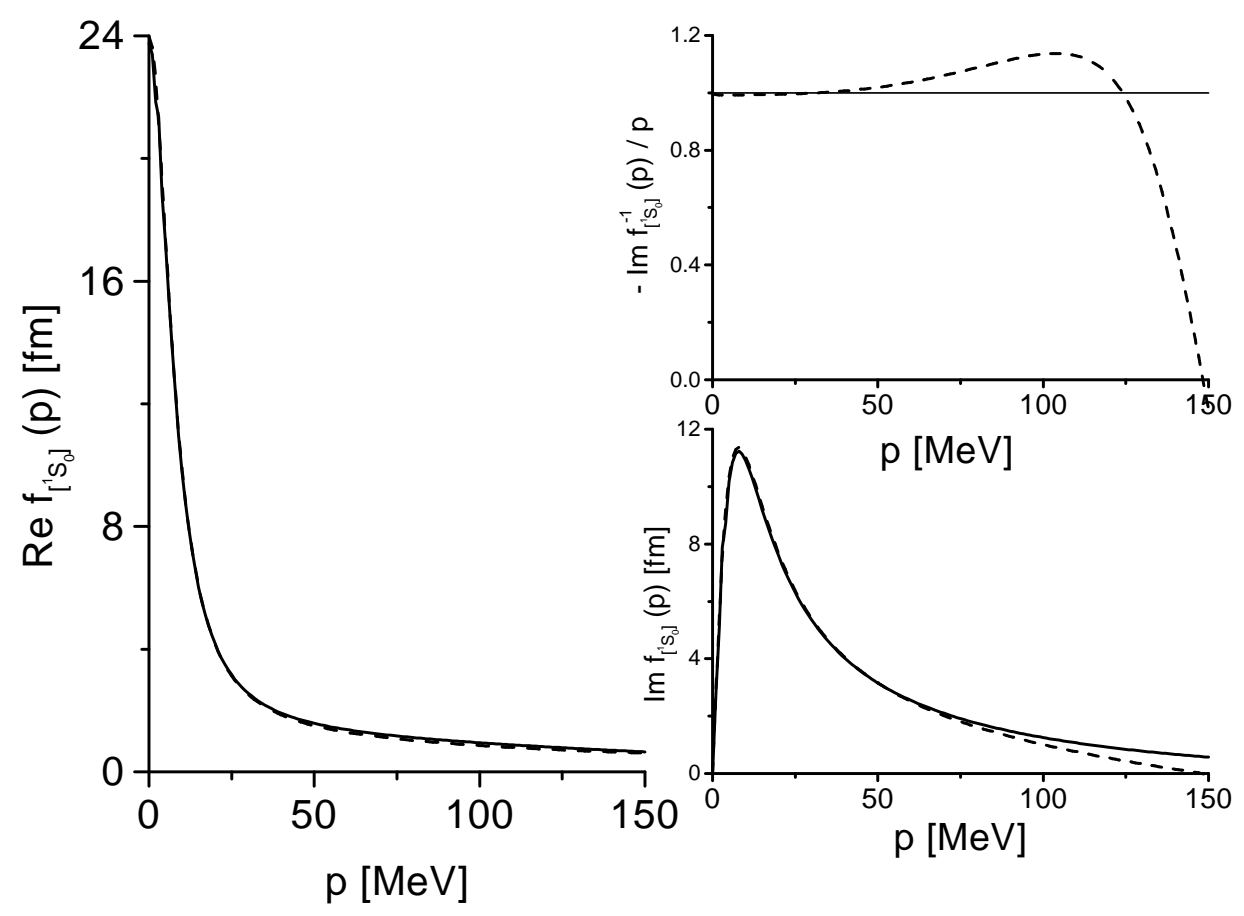

Fig. 2. The left hand figure shows real part of the np-scattering amplitude $f_{\left[{ }^{1} S_{0}\right]}(p)=\sqrt{s} T_{\left[{ }^{1} S_{0}\right]}(p) /(8 \pi)$. The right hand figure shows imaginary part of the np-scattering amplitude $\Im f_{\left[{ }^{1} S_{0}\right]}(p)$ and the unitarity function $-\Im f_{\left[{ }^{1} S_{0}\right]}^{-1}(p) / p$, which is one if unitarity holds. The solid lines are from the PWA93 solution of [4] and the dashed lines are the result of the first order CE scheme with $\zeta_{2}^{(0)}=-1.363 \mathrm{fm}$, $\zeta_{0}^{(2)}=1.771 \mathrm{fm}$ and $z_{0}=-45.68 \mathrm{MeV}($ see $(\mathrm{C} .8))$.

$$
b_{\text {eff }}^{(1)}=-\frac{z_{0} m}{\pi m_{\pi}^{2}} g_{A}^{2} \lambda^{2}\left(1+\frac{16}{5} \frac{z_{0}}{m_{\pi}}+\mathcal{O}\left(\frac{z_{0}^{2}}{m_{\pi}^{2}}\right)\right)+\mathcal{O}(Q)
$$

and compare it with the result from effective range theory (see (29)). We conclude that (103) predicts the wrong sign of this term and therewith confirm our result that (99) is in strong conflict with the unitarity condition

$$
\Im T_{\left[{ }^{1} S_{0}\right]}^{-1}(p)=-\frac{1}{2} \Im J_{R}\left(p, z_{0}\right)
$$

In appendix $\mathrm{C}$ the reader may find details of the NNLO terms in the CE scheme. In Fig 2 we present the result of a low energy fit. The description of the scattering amplitude is somewhat improved for $p<100 \mathrm{MeV}$ as compared to the zeroth order result. In particular the unitarity condition is met to better accuracy. However the first order calculation clearly signals the breakdown of the CE scheme at momenta $p \simeq 150 \mathrm{MeV}$. To be explicit: at the chiral orders we are working here (see (65)) the strength of the effective pole at $p=i \bar{z}$ 
(see (30)) must be represented by the pion-nucleon box function $R_{\pi \pi}(p)$ (see (80)) since it is the only function showing a branch point in the upper complex half plane at $p=i m_{\pi} \sim i \bar{z}$. Recall here that all vertex functions and bubble functions have branch cuts only on the lower complex half plane. Since the strength of the cut in $R_{\pi \pi}(p)$ is much too weak to represent the strength of the effective pole at $p=i \bar{z}$ the CE scheme fails for $p \ll \bar{z} \simeq 150 \mathrm{MeV}$. Note also that the parameters $\zeta_{0}^{(0)}$ and $z_{0}$ of the zeroth order and first order calculation are strongly renormalized. This points at the poor convergence properties of the CE scheme even at low momenta $p<150 \mathrm{MeV}$. This could have been anticipated by inspecting the ratio of the renormalized functions

$$
g_{A}^{2} \lambda^{2} \frac{J_{\pi, R}(0, z)}{J_{R}(0, z)}=g_{A}^{2} \lambda^{2} \frac{m m_{\pi}}{2 \pi}+\mathcal{O}(z) \simeq 0.9
$$

at $p=0$. From our calculation we conclude that in fact we have to change scheme and reformulate our effective field theory along the lines already anticipated. We emphasize that the result (105) does not imply that successive unitary iterations of $K_{\pi, S}$ need to be summed to all orders. As shown before here typical ratios are well behaved (see $(81,82)$ ). The ratio $(105)$ is to be taken as further evidence that the properly renormalized denominator (67) must not be expanded.

We close this section by a comment on the so called KSW scheme proposed by Kaplan, Savage and Wise [15]. In the KSW scheme the scattering phase $\delta_{\left[{ }^{[} S_{0}\right]}(p)$ is expanded perturbatively in the pion dynamics imposing the correct pseudo-bound state structure. It followed from our CE scheme upon a further expansion. For independent calculations in the KSW scheme at subleading orders see [31]. In our work we insist on the CE scheme since the unitarity condition (104) provides a powerful consistency check for the CE scheme which is obscured in the KSW scheme. If the CE scheme showed good convergence properties it would be equivalent to the KSW scheme. The rather poor convergence properties of the CE scheme necessarily implies that also the KSW scheme is poorly convergent. We conclude therefore that neither the $\mathrm{CE}$ nor the KSW scheme provides an improvement upon the effective range theory. Furthermore note that by expanding the phase shift directly rather than the amplitude makes it more difficult to detect a potential breakdown of the scheme. This is clearly demonstrated by the effective range theory. Here the second low momentum pole at $p=i \bar{z}$ (see $(27,30)$ ) restricts the applicability of the CE and the KSW scheme to momenta $p<|\bar{z}|$. The breakdown is triggered by a 'weak' cut singularity in the KSW scheme as compared to a 'strong' pole singularity in the CE scheme:

$$
\delta_{\left[{ }^{1} S_{0}\right]}(p)=\frac{\pi}{2}-\arctan \left(-\frac{1}{a p}+\frac{1}{2} r p\right) .
$$


In the KSW scheme the branch cut of the arctan function at $\pm i$ leads to the convergence bound $p<|\bar{z}|=|2 / r|+\mathcal{O}(1 / a)$. We conclude that in order to uncover the physical significance of non-perturbative strength at $p \simeq i \bar{z}$ it is advantageous to choose the CE scheme.

\subsection{Unitary chiral expansion (UCE) scheme}

In the previous sections we collected mounting evidence that the denominator in (38) or (79) must not be expanded further. We have demonstrated that the CE scheme, based on such an expansion, is not applicable in the spin singlet channel due to poor convergence properties. In this central section of this work our new scheme already outlined in section 2.4 is constructed explicitly. It shows much improved convergence properties and comprises unitarity exactly. Formally the scheme arises if the renormalized coupling, $g_{R}^{-1}\left(p, m_{\pi}\right)$, changes according to (94) its chiral power from plus two at very small momenta $p \ll$ $\mu_{\sigma}$ to zero at intermediate small momenta $p \sim \mu_{\sigma}$. As a consequence the denominator in (79) must not be expanded if the effective field theory is to be applied for momenta $p<\mu_{\sigma}+\Lambda_{\text {nat. }}$. Keeping the denominator on the one hand takes care of the proper chiral power of the renormalized coupling at intermediate small momenta $p \sim \mu_{\sigma}$ and on the other hand induces a particular resummation for the low momentum regime which greatly improves its convergence property. We refer to this approach as the 'UCE'-scheme since, as we will elaborate on in detail below, it in addition leads to exact unitarity.

The UCE scheme should be viewed as a systematic summation of the CE scheme (see (94)). The renormalization procedure is identical to the renormalization procedure of the CE scheme: all vertex functions $V_{\pi^{n}, S}(p)$ are renormalized by appropriate 'loop' subtractions induced by a properly constructed local subtraction kernel $\bar{K}_{S}$. We emphasize again that this procedure leads to renormalization scale independent results for the nth order vertex functions $V_{\pi^{n}, R}(p)$ and the nth order box functions $R_{\pi^{n}}(p)$. The remaining divergencies of the nth order bubble functions $J_{\pi^{n}, S}(p)$ are renormalized by a subtraction procedure at $p=-i z_{0}$. Thus the renormalized bubble functions $J_{\pi^{n}, R}\left(p, z_{0}\right)$ show an explicit dependence on the subtraction point $z_{0}$ or more generally they depend on some renormalization scale. At strictly $K_{S}=\bar{K}_{S}$ we obtain all together renormalization scale independent results since the renormalization scale can be absorbed into the renormalized coupling function $g_{R}\left(p, m_{\pi}\right)$. As advocated in section 2.4 it is advantageous to exploit the freedom to not strictly require $K_{S}=\bar{K}_{S}$. For the CE scheme we have established that the scattering amplitude evaluated to a given chiral order is independent on $\left(K_{S}-\bar{K}_{S}\right)^{n}$ if we count $K_{S}-\bar{K}_{S} \sim Q$. In the UCE scheme this does not hold anymore since it is an infinite resummation of the $\mathrm{CE}$ scheme. The residual dependence on $\left(K_{S}-\bar{K}_{S}\right)^{n}$ is fixed as to implement unitarity exactly at given order in the expansion. In 
order not to spoil the renormalization procedure the residual dependence on $\left(K_{S}-\bar{K}_{S}\right)^{n}$ 'acts' exclusively on finite parts as defined in (47) and (48). This amounts to dropping all divergent terms proportional to $(c-1 / 2) J\left(-i z_{0}\right)$ in the loop functions (70). More explicitly we arrive at:

$$
\begin{aligned}
T_{\left[{ }^{1} S_{0}\right]}(p) & =2 g_{A}^{2} \lambda^{2}\left(R_{\pi}(p)+c-\frac{1}{2}\right) \\
& +\frac{2+4 g_{A}^{2} \lambda^{2} \bar{V}_{\pi, S}\left(p, z_{0}\right)}{g_{R}^{-1}\left(p, m_{\pi}\right)-J_{R}\left(p, z_{0}\right)-g_{A}^{2} \lambda^{2} \bar{J}_{\pi, S}\left(p, z_{0}\right)}
\end{aligned}
$$

with the renormalized loop functions

$$
\begin{aligned}
& \bar{V}_{\pi, S}\left(p, z_{0}\right)=\left(c-\frac{1}{2}\right) J_{R}\left(p, z_{0}\right)+V_{\pi, R}(p), \\
& \bar{J}_{\pi, S}\left(p, z_{0}\right)=\left(c-\frac{1}{2}\right) J_{R}^{2}\left(p, z_{0}\right)+J_{\pi, R}\left(p, z_{0}\right) .
\end{aligned}
$$

The renormalized coupling $g_{R}\left(p, m_{\pi}\right)$ is given by (94) and the loop functions in $(71,80)$. Note that according to our definition (48) and (91) the finite 'part' of the auxiliary coupling function $g_{H}\left(s, m_{\pi} ; c(s)\right)$ of $(89)$ must be identified with the renormalized coupling $g_{R}\left(p, m_{\pi}\right)$. The function $c(s)$ is now unambiguously determined by the unitarity constraint. According to (49) the function $c(s)$, representing a separable interaction, is constructed such that the s-wave and on-shell projection of $T_{\pi, S}$ vanishes at the given order

$$
\left\langle T_{\pi, S}\right\rangle=2 g_{A}^{2} \lambda^{2}\left(R_{\pi}(p)+c-\frac{1}{2}\right)=0+\mathcal{O}(Q) .
$$

We find that the unique choice satisfying (109)

$$
c(s)=\frac{1}{2}-R_{\pi}(p)+\mathcal{O}(Q)
$$

renders the scattering amplitude (107) unitary. Note that the subtraction function $c(s)-1 / 2$ now carries chiral order zero. This does not contradict the L-counting rule because the subtraction $c(s)$ is now only active on convergent parts of the loops. We emphasize that the construction (110) holds irrespective of the form of the renormalized coupling $g_{R}\left(p, m_{\pi}\right)$.

The mechanism which leads to a unitary scattering amplitude is readily understood. The vertex loop function $V_{\pi, R}(p)$, the bubble function $J_{\pi, R}\left(p, z_{0}\right)$, and the one-pion exchange contribution $R_{\pi}(p)$ are not independent entities. They are interrelated by causality. Cutkosky's cutting rules [23] lead to the identities

$$
R_{\pi}(p) \Im J_{R}\left(p, z_{0}\right)=\Im V_{\pi, R}(p)
$$




$$
2\left(\Re V_{\pi, R}(p)\right)\left(\Im J_{R}\left(p, z_{0}\right)\right)=\Im J_{\pi, R}\left(p, z_{0}\right)
$$

which are consistent with our analytic results $(71,80)$ and directly confirm the unitarity condition (104). As a consequence the phase shift can now be identified unambiguously

$$
p \cot \delta_{\left[{ }^{1} S_{0}\right]}(p)=\frac{4 \pi}{\sqrt{s}} \frac{\bar{g}_{R}^{-1}\left(p, m_{\pi}\right)-g_{A}^{2} \lambda^{2} \Re \bar{J}_{\pi, S}\left(p, z_{0}\right)}{1+2 g_{A}^{2} \lambda^{2} \Re \bar{V}_{\pi, S}\left(p, z_{0}\right)}
$$

where we introduced

$$
\bar{g}_{R}^{-1}\left(p, m_{\pi}\right)=g_{R}^{-1}\left(p, m_{\pi}\right)-\Re J_{R}\left(p, z_{0}\right)
$$

for notational convenience.

We point out that any further expansion of (112) is necessarily in conflict with unitarity (or even worse with the complicated low energy structure of the scattering amplitude) since it would invalidate the derivation of our result (112). Finally it is instructive to consider the limit of a vanishing renormalized coupling function: $g_{R}\left(p, m_{\pi}\right)=0$ leads to a zero phase shift $\delta_{\left[{ }^{1} S_{0}\right]}(p)=0$. This demonstrates that the renormalized coupling function $g_{R}$ does not only represent the physics of short range repulsion ( $\omega$-exchange) and intermediate range attraction ( $\sigma$-exchange) it also encompasses some residual pion dynamics. The important achievement lies in the fact that $g_{R}$ does not pick up pion induced singularities. In other words $g_{R}$ does not exhibit branch points at $p= \pm n i m_{\pi} / 2$ with $n=1,2,3, \ldots$ and therefore $g_{R}$ is expected to respect a Taylor expansion with the typical scale set by a rather large $\Lambda_{\text {nat. }} \sim 600 \mathrm{MeV}$.

\subsection{NNLO terms of UCE scheme}

We turn to the next terms in our expansion. Again all divergent terms in the loop functions $(70,74)$ are dropped according to the argument given in the previous section. We derive

$$
\begin{aligned}
T_{\left[{ }^{1} S_{0}\right]}(p) & =2 g_{A}^{2} \lambda^{2}\left(R_{\pi}(p)+c-\frac{1}{2}\right)+2 g_{A}^{4} \lambda^{4}\left(c-\frac{1}{2}\right)^{2} J_{R}\left(p, z_{0}\right) \\
& +4 g_{A}^{4} \lambda^{4}\left(c-\frac{1}{2}\right) V_{\pi, R}(p)+2 g_{A}^{4} \lambda^{4} R_{\pi \pi}(p) \\
& +\frac{2\left(1+g_{A}^{2} \lambda^{2} \bar{V}_{\pi, S}\left(p, z_{0}\right)\right)^{2}+4 g_{A}^{4} \lambda^{4} \bar{V}_{\pi \pi, S}\left(p, z_{0}\right)}{g_{R}^{-1}(p)-J_{R}\left(p, z_{0}\right)-g_{A}^{2} \lambda^{2} \bar{J}_{\pi, S}\left(p, z_{0}\right)-g_{A}^{4} \lambda^{4} \bar{J}_{\pi \pi, S}\left(p, z_{0}\right)}
\end{aligned}
$$

with the renormalized loop functions 


$$
\begin{aligned}
\bar{V}_{\pi \pi, S}\left(p, z_{0}\right) & =V_{\pi \pi, R}(p)+\left(c-\frac{1}{2}\right)^{2} J_{R}^{2}\left(p, z_{0}\right) \\
& +\left(c-\frac{1}{2}\right)\left(J_{R}\left(p, z_{0}\right) V_{\pi, R}(p)+J_{\pi, R}\left(p, z_{0}\right)\right) \\
\bar{J}_{\pi \pi, S}\left(p, z_{0}\right) & =J_{\pi \pi, R}\left(p, z_{0}\right)+\left(c-\frac{1}{2}\right)^{2} J_{R}^{3}\left(p, z_{0}\right) \\
& +2\left(c-\frac{1}{2}\right) J_{R}\left(p, z_{0}\right) J_{\pi, R}\left(p, z_{0}\right) .
\end{aligned}
$$

Before constructing the subtraction function $c(s)$ at subleading order we collect the relevant causality identities predicted by Cutkosky's [23] cutting rules:

$$
\begin{aligned}
\Im R_{\pi \pi}(p) & =R_{\pi}^{2}(p) \Im J_{R}\left(p, z_{0}\right), \\
\Im V_{\pi \pi, R}(p) & =\left(\Re V_{\pi, R}(p)\right)\left(\Im V_{\pi, R}(p)\right)+\left(\Re R_{\pi \pi}(p)\right)\left(\Im J_{R}\left(p, z_{0}\right)\right), \\
\Im J_{\pi \pi, R}\left(p, z_{0}\right) & =2\left(\Re V_{\pi \pi, R}(p)\right)\left(\Im J_{R}\left(p, z_{0}\right)\right)+\left|V_{\pi, R}(p)\right|^{2} \Im J_{R}\left(p, z_{0}\right) .(11
\end{aligned}
$$

Again the identities (116) are respected by our analytic results $(76,80)$. The subleading term of the subtraction function $c(s)$ can now be constructed by the requirement

$$
\begin{aligned}
\left\langle T_{\pi, S}\right\rangle & =2 g_{A}^{2} \lambda^{2}\left(R_{\pi}(p)+c-\frac{1}{2}\right)+2 g_{A}^{4} \lambda^{4}\left(c-\frac{1}{2}\right)^{2} J_{R}\left(p, z_{0}\right) \\
& +4 g_{A}^{4} \lambda^{4}\left(c-\frac{1}{2}\right) V_{\pi, R}(p)+2 g_{A}^{4} \lambda^{4} R_{\pi \pi}(p) \\
& =0+\mathcal{O}\left(Q^{2}\right)
\end{aligned}
$$

that the s-wave and on-shell projection of $T_{\pi, S}$ vanishes to the given order (49). The solution of (117) is now readily found with

$$
\begin{aligned}
c(s) & =\frac{1}{2}-R_{\pi}(p)-g_{A}^{2} \lambda^{2} \Re R_{\pi \pi}(p)-g_{A}^{2} \lambda^{2} R_{\pi}^{2}(p) \Re J_{R}\left(p, z_{0}\right) \\
& +2 g_{A}^{2} \lambda^{2} R_{\pi}(p) \Re V_{\pi, R}(p)+\mathcal{O}\left(Q^{2}\right) .
\end{aligned}
$$

Explicit calculation indeed confirms that with (118) the s-wave scattering amplitude (114) satisfies unitarity. The phase shift can be identified unambiguously and we finally arrive at the central result of our work:

$$
p \cot \delta_{\left[{ }^{1} S_{0}\right]}(p)=\frac{4 \pi}{\sqrt{s}} \frac{\bar{g}_{R}^{-1}(p)-g_{A}^{2} \lambda^{2} \Re \bar{J}_{\pi, S}\left(p, z_{0}\right)-g_{A}^{4} \lambda^{4} \Re \bar{J}_{\pi \pi, S}\left(p, z_{0}\right)}{\Re\left(1+g_{A}^{2} \lambda^{2} \bar{V}_{\pi, S}\left(p, z_{0}\right)\right)^{2}+2 g_{A}^{4} \lambda^{4} \Re \bar{V}_{\pi \pi, S}\left(p, z_{0}\right)} .
$$

Note that in (119) the numerator has to be evaluated at chiral order three and the denominator at chiral order two. In other words in $\bar{J}_{\pi, S}(p)$ the subtraction function $c(s)$ has to be included to chiral order one as given by (118) whereas 
in $\bar{J}_{\pi \pi, S}(p)$ the subtraction function $c(s)$ is to be included to chiral order zero as given by (110).

\section{Results and discussion}

We confront our unitary chiral expansion (UCE) with the partial wave analysis (PWA93) of the Nijmegen group [4]. Our result at order $Q^{0}$ is given in $(108,110,112)$ and at order $Q^{1}$ in $(115,118,119)$. At leading chiral orders we encounter the renormalized coupling function

$$
g_{R}^{-1}\left(p, m_{\pi}\right)=\frac{m}{2 \pi}\left(\xi_{\sigma} \frac{p^{2}+z_{0}^{2}}{p^{2}-\mu_{\sigma}^{2}}+\xi_{2}^{(0)}\left(p^{2}+z_{0}^{2}\right)+m_{\pi}^{2} \xi_{0}^{(2)}\right)+\mathcal{O}\left(Q^{4}\right)
$$

characterized by five free parameters $\xi_{\sigma}, \xi_{2}^{(0)}, \xi_{0}^{(2)}, \mu_{\sigma}$ and $z_{0}$. Here we absorb the finite contribution $m_{\pi}^{3} \xi_{0}^{(3)}$ into $m_{\pi}^{2} \xi_{0}^{(2)}$ for convenience. Our parameters are adjusted to reproduce the ${ }^{1} S_{0}$-partial wave in the pn-channel. With $g_{\pi}=$ $g_{A}^{2} m m_{\pi} /\left(16 \pi f_{\pi}^{2}\right)=0.451, m_{\pi}=139.0 \mathrm{MeV}$ and $m=938.9 \mathrm{MeV}$ we perform a least square fit to the single energy ${ }^{1} S_{0}$ partial wave as given in [4] with finite error bars. Tab. 1 presents the resulting parameters together with the $\chi^{2}$-value of the fit. We observe that the $\chi^{2}$ of the UCE result at order $Q^{1}$ is significantly improved upon the $\chi^{2}$ of the UCE result at order $Q^{0}$ although there is no additional free parameter involved. The parameters are only weakly renormalized indicating good convergence properties and consistency with the naturalness assumption (see (26)). Moreover we confirm that the inclusion of pionic effects leads to a much better description of the partial wave. The $\chi^{2}$ is reduced by a factor of 5 from 25 to 5 . The collum in Tab. 1 labeled 'no pions' refers to our calculation with $g_{A}=0$.

We emphasize that the parameter $z_{0}$ has a physical interpretation. It represents the pseudo-bound state pole position in the chiral limit with $m_{\pi}=0$. The chiral limit leads in fact to a real bound state at $p \simeq i 37 \mathrm{MeV}$ with binding energy $\epsilon \simeq z_{0}^{2} / m \simeq 1.5 \mathrm{MeV}$ rather than a pseudo-bound state obtained with $m_{\pi}>0$. Our result demonstrates that the effective range parameter, $r\left({ }^{1} S_{0}\right)$, defined in (28), is driven by the physical scale $\mu_{\sigma}$ rather than the pion mass as one might expect naively. In the language of the $\sigma-, \omega$-meson exchange picture we would say that the effective range is mainly determined by the subtle interplay of intermediate attraction and short range repulsion rather than by the pion exchange. The parameter $\mu_{\sigma}$ determines the zero of the phase shift and $\zeta_{\sigma}$

$$
\delta_{\left[{ }^{1} S_{0}\right]}(p) \simeq \frac{p^{2}-\mu_{\sigma}^{2}}{\xi_{\sigma} \mu_{\sigma}}
$$


the behavior close to $p \simeq \mu_{\sigma}$. In (121) we omitted pion induced terms for simplicity. We point out that the dynamic chiral power of our renormalized coupling function $g_{R}$ in (120) with $g_{R}^{-1} \sim Q^{2}$ for $p \ll \mu_{\sigma}$ but $g_{R}^{-1} \sim Q^{-2}$ for $p \simeq \mu_{\sigma}$ prohibits any further expansion of the scattering amplitude beyond (114) if the theory is to be applied for momenta $0<p<m_{\omega}$. Our phase shifts are given in Tab. 2. where for the reader's convenience we also included results of two modern potential models as given in $[32,33]$. The latter potentials reproduce the empirical phase shift rather well, where the Bonn potential shows a somewhat better $\chi^{2}$ as compared to the Nijm93 potential. We conclude that our chiral description of the ${ }^{1} S_{0}$-phase shift does compete in quality with modern nucleon-nucleon potentials.

It is instructive to discuss the singularity structure of our scattering amplitude (114). In Fig. 3 we show the scattering amplitude $f_{\left[{ }^{1} S_{0}\right]}(i q)$ evaluated below threshold with $p=i q+\epsilon$. The pseudo-bound state pole at $q \simeq-8 \mathrm{MeV}$ and the anomalous thresholds at $q= \pm m_{\pi} / 2$ are clearly seen. For unphysical negative $q \ll-8 \mathrm{MeV}$ (2nd Rieman sheet) the amplitude is rather smooth whereas for positive $q$ (1st Rieman sheet) the amplitude shows a rather complex structure anticipated in our discussion of section 2.3. The figure shows a well defined peak structure in the imaginary part of the amplitude and a corresponding zero of the real part at $p \simeq i 225 \mathrm{MeV}$, which was roughly predicted by the effective range theory (see (30)). From an analyticity point of view it is now obvious that a successful effective field theory description of the ${ }^{1} S_{0}$-partial wave amplitude requires the incorporation of this singularity. This explains the failure of the CE scheme (see section 3.3) which includes the pseudo-bound state pole but misses to describe the complete singularity structure seen in Fig. 3.

In Fig. 4 we show how our UCE- $Q^{1}$ fit to the ${ }^{1} S_{0}$ phase shift extrapolates

\begin{tabular}{|r||c|c|c|}
\hline & no pions & UCE- $Q^{0}$ & UCE- $^{1}$ \\
\hline \hline$\chi_{\text {PWA93 }}^{2}$ & 25.3 & 12.9 & 5.3 \\
\hline$a\left({ }^{1} S_{0}\right)[\mathrm{fm}]$ & -23.33 & -23.48 & -23.59 \\
\hline$r\left({ }^{1} S_{0}\right)[\mathrm{fm}]$ & 2.54 & 2.60 & 2.64 \\
\hline$\xi_{2}^{(0)}[\mathrm{fm}]$ & 0.391 & 0.451 & 0.451 \\
\hline$\xi_{0}^{(2)}[\mathrm{fm}]$ & 0 & 0.6964 & 0.7490 \\
\hline$\xi_{\sigma}[\mathrm{MeV}]$ & -558.9 & -672.5 & -724.1 \\
\hline$\mu_{\sigma}[\mathrm{MeV}]$ & 354.1 & 353.8 & 353.5 \\
\hline$z_{0}[\mathrm{MeV}]$ & 8.04 & -45.84 & -37.04 \\
\hline
\end{tabular}

Table 1

Set of parameters obtained by fitting the ${ }^{1} S_{0}(\mathrm{np})$ phase shift of PWA93. 


\begin{tabular}{|r||c|c|c|c|c|c|}
\hline$E_{\text {lab }}[\mathrm{MeV}]$ & PWA93 & no pions & UCE- $Q^{0}$ & UCE- $Q^{1}$ & Bonn & Nijm93 \\
\hline \hline 1 & $62.07 \pm 0.03$ & 62.06 & 62.06 & 62.06 & 62.12 & 62.07 \\
\hline 5 & $63.63 \pm 0.08$ & 63.86 & 63.79 & 63.73 & 63.67 & 63.62 \\
\hline 10 & $59.96 \pm 0.11$ & 60.03 & 60.04 & 60.02 & 59.91 & 59.94 \\
\hline 25 & $50.90 \pm 0.19$ & 50.46 & 50.63 & 50.75 & 50.82 & 50.86 \\
\hline 50 & $40.54 \pm 0.28$ & 39.83 & 40.02 & 40.20 & 40.44 & 40.38 \\
\hline 100 & $26.78 \pm 0.38$ & 26.43 & 26.45 & 26.53 & 26.27 & 26.17 \\
\hline 150 & $16.94 \pm 0.41$ & 17.08 & 16.98 & 16.96 & 16.17 & 15.71 \\
\hline 200 & $8.94 \pm 0.39$ & 9.36 & 9.24 & 9.15 & 8.14 & 7.07 \\
\hline 250 & $1.96 \pm 0.37$ & 2.35 & 2.27 & 2.19 & 1.40 & -0.45 \\
\hline 300 & $-4.46 \pm 0.43$ & -4.45 & -4.42 & -4.44 & -4.45 & -7.18 \\
\hline 350 & $-10.59 \pm 0.62$ & -11.30 & -11.13 & -11.02 & -9.65 & -13.32 \\
\hline
\end{tabular}

Table 2

We confront our result (112) and (119) with the PWA93 solution for the ${ }^{1} S_{0}(n p)$ partial wave. The $\chi^{2}$ of (112) with 12.9 and of (119) with 5.7 is significantly improved as compared to the $\chi^{2}$ of 25.3 which follows if pion effects are switched off (i.e. $\left.g_{A}=0, m_{\pi}=0\right)$. The set of parameters is given in Tab. 1 . The $\chi^{2}$ are 37.0 for Nijm93 and 12.2 for the Bonn potential. Note that here we omitted the first point at $E_{\mathrm{lab}}=1 \mathrm{MeV}$ since its inclusion would somewhat artificially worsen the $\chi^{2}$.

to higher energies. Here we confront our result with Arndt's SP99 solution [33]. We observe that in fact our UCE scheme appears better compatible with the PWA93 solution of the Nijmegen group [32]. Note that the zeroth order (dashed line) and first order (solid line) results give very similar predictions for the phase shift for a given set of parameters confirming good convergence properties of our UCE scheme. The main effect of the correction terms sit at low momenta $p<200 \mathrm{MeV}$. Most dramatic is the modification at low momenta if the pion effects are switched off with $g_{A}=0$ as demonstrated with the dotted line in Fig. 4. Note that of course a set of renormalized parameters leads to a better description of the phase shift even with $g_{A}=0$ as is shown in Tab. 2. Our full result (solid line) starts to deviate significantly from the SP99 solution for momenta $p>450$ somewhat above the pion production threshold at $p \simeq 370 \mathrm{MeV}$.

We conclude our work with a short explorative discussion of the next terms in our expansion. At order $Q^{2}$ one needs to include the irreducible two-pion exchange diagrams in the interaction kernel $K_{\pi}$ studied in $[8,9,34]$ :

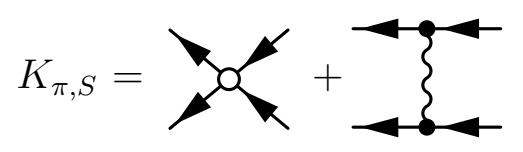



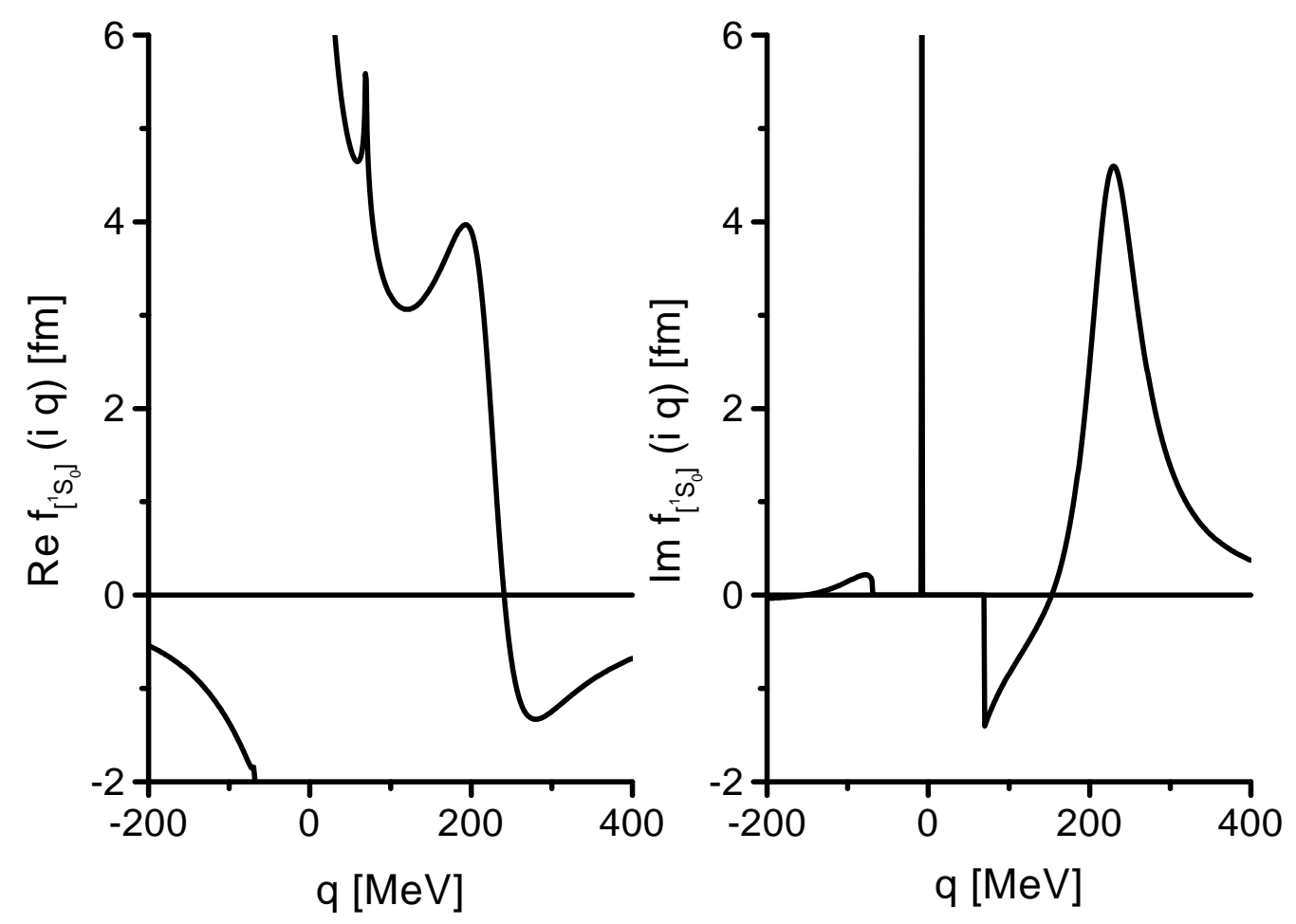

Fig. 3. The ${ }^{1} S_{0}$-partial wave amplitude $f_{\left[{ }^{1} S_{0}\right]}(i q)=\sqrt{s} T_{\left[{ }^{1} S_{0}\right]}(i q) /(8 \pi)$ of $(114)$ evaluated with the UCE- $Q^{1}$ parameters as given in Tab. 1 .

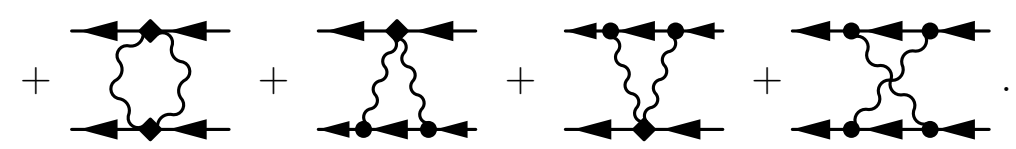

The scattering amplitude $T_{\pi, S}$ is to be evaluated up to order $Q^{2}$ applying the L-counting rule. This amounts at most a thrice iterated kernel $K_{\pi, S}$. Moreover $1 / m$ correction terms need to be included. Also the interaction $K_{\sigma, S}$ receives a contribution

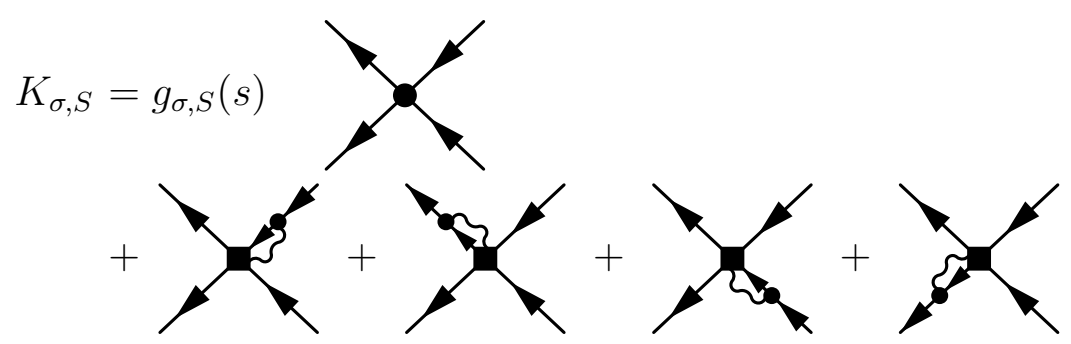

from a new chiral vertex

$$
\mathcal{L}=\frac{1}{4} g^{(\pi)} \lambda^{4}\left(\bar{N} \gamma_{5} C \vec{\tau} \tau_{2} \bar{N}^{t}\right)\left(N^{t} C^{-1} \tau_{2}(\not \partial \vec{\pi}) N\right)+\text { h.c. }
$$




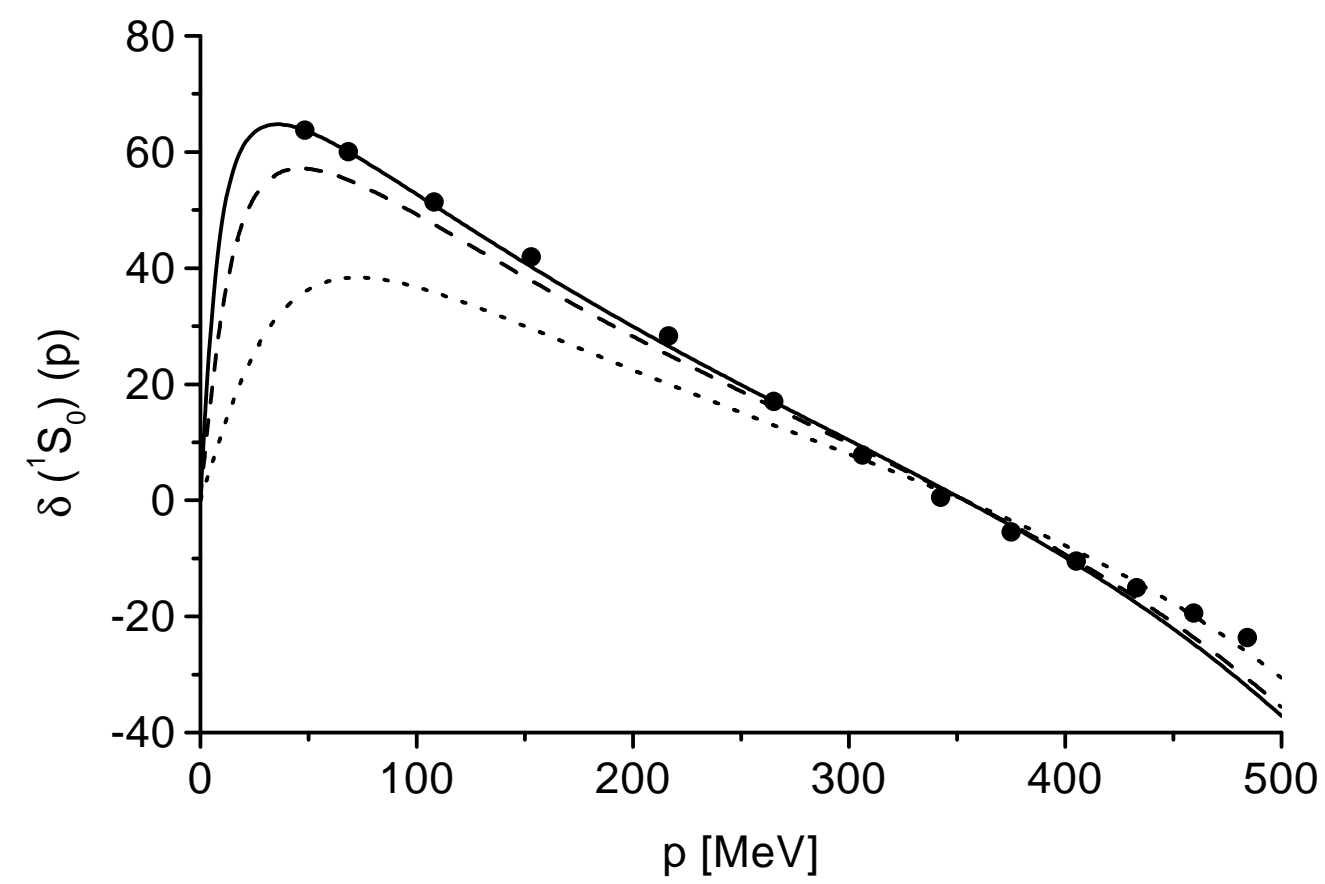

Fig. 4. The ${ }^{1} S_{0}$-phase shift evaluated with the UCE parameters at order $Q^{1}$ as given in Tab. 1. The solid line is the full result $\left(\mathrm{UCE}-Q^{1}\right)$ obtained from the fit to the PWA93 solution. The dashed line is the zero order result of the UCE scheme and the dotted line follows from the UCE scheme with $g_{A}=0$ (no pions). The dots represent the SP99 analysis of Arndt [33].

part of the chiral Lagrangian. The pion production parameter $g^{(\pi)}$ is unconstrained by chiral symmetry and may be estimated by meson-exchange phenomenology. We point out that the interaction $K_{\sigma, S}$ receives only 2 -nucleon irreducible contributions which are also irreducible with respect to pion lines. This is a crucial element of our UCE scheme which only then leads to a renormalized inverse coupling function $g_{R}^{-1}\left(p, m_{\pi}\right)$ free of any pionic cut structure. Moreover the two-nucleon propagator $G$ in $(5,31)$ is corrected by the leading nucleon self energy loop diagrams:

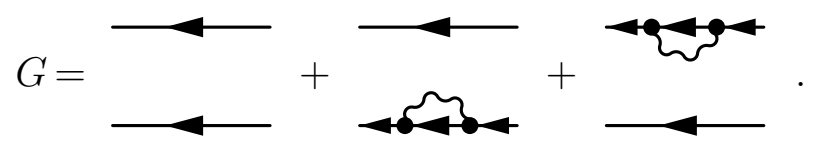

The important new element of the order $Q^{2}$ calculation are the pion production cuts of loop functions. They give rise to a complex phase shift for $p>\mu_{\pi}$ with the pion production scale $\mu_{\pi}^{2}=m m_{\pi}+m_{\pi}^{2} / 4$. We estimate the result of the UCE- $Q^{2}$ calculation by adding all pion production contributions of order $Q^{2}$ to our $\mathrm{UCE}^{-\mathrm{Q}^{1}}$ result (114). At the given order there are three different sources for pion production. First there is the pion production cut of the two-loop bubble diagram $J_{\pi, R}^{(\pi)}\left(p, z_{0}\right)$ (see $(68)$ ) with 


$$
\begin{aligned}
J_{\pi, R}^{(\pi)}\left(p, z_{0}\right) & =\frac{1}{g_{A}^{2} \lambda^{2}} \\
& =\frac{m_{\pi}^{4}}{(2 \pi)^{4}}\left(-\frac{\mu_{\pi}^{2}}{p^{2}}+\frac{\left(p^{2}-\mu_{\pi}^{2}\right)^{2}}{p^{4}} \ln \left|\frac{\mu_{\pi}^{2}}{\mu_{\pi}^{2}-p^{2}}\right|\right. \\
& -\frac{\mu_{\pi}^{2}}{z_{0}^{2}}-\frac{\left(z_{0}^{2}+\mu_{\pi}^{2}\right)^{2}}{z_{0}^{4}} \ln \left|\frac{\mu_{\pi}^{2}}{\mu_{\pi}^{2}+z_{0}^{2}}\right| \\
& \left.+i \pi \Theta\left(p-\mu_{\pi}\right) \frac{\left(p^{2}-\mu_{\pi}^{2}\right)^{2}}{p^{4}}\right)+\mathcal{O}\left(Q^{5}\right)
\end{aligned}
$$

We wish to emphasize that the chiral expansion of the pion production terms is not performed applying the heavy baryon technique since it would destroy the proper pion production threshold. Rather we expand the spectral density in powers of $\left(p^{2}-\mu_{\pi}^{2}\right)^{n}$ identifying $\mu_{\pi} \sim Q$ as a further characteristic small scale. This procedure keeps the correct pion production threshold. Here we encounter a clear advantage of the relativistic chiral Lagrangian over its heavy baryon representation.

We turn to the second pion production term. It results from the nucleon self energy correction of the one-loop bubble

$$
J_{\Sigma, R}^{(\pi)}\left(p, z_{0}\right)=\frac{1}{g_{A}^{2} \lambda^{2}}
$$

with its leading contribution proportional to the expression found for the first term (126) with $J_{\Sigma, R}^{(\pi)}\left(p, z_{0}\right)=3 J_{\pi, R}^{(\pi)}\left(p, z_{0}\right)$. We point out that the dominant term at order $Q^{2}$ arises from the pion production vertex (124) which induces the loop function:

$$
\begin{aligned}
J_{V, R}^{(\pi)}\left(p, z_{0}\right) & =\frac{\lambda^{-5}}{g_{A} g^{(\pi)}} \\
& =2 \frac{m_{\pi}^{4} p^{2}}{(2 \pi)^{4} m}\left(\frac{\left(p^{2}-\mu_{\pi}^{2}\right)^{2}}{p^{4}} \ln \left|\frac{\mu_{\pi}^{2}}{\mu_{\pi}^{2}-p^{2}}\right|+\frac{\left(z_{0}^{2}+\mu_{\pi}^{2}\right)^{2}}{z_{0}^{2} p^{2}} \ln \left|\frac{\mu_{\pi}^{2}}{\mu_{\pi}^{2}+z_{0}^{2}}\right|\right. \\
& +i \pi \Theta\left(p-\mu_{\pi}\right)
\end{aligned}
$$


Technically the three pion production loop functions are incorporated easily by the formal replacement in (114):

$$
\begin{aligned}
g_{R}^{-1} & \rightarrow g_{R}^{-1}-g_{A}^{2} \lambda^{2} J_{\pi, R}^{(\pi)}\left(p, z_{0}\right)-g_{A}^{2} \lambda^{2} J_{\Sigma, R}^{(\pi)}\left(p, z_{0}\right) \\
& -g_{R}^{-1} g_{A} g^{(\pi)} \lambda^{5} J_{V, R}^{(\pi)}\left(p, z_{0}\right) .
\end{aligned}
$$

We point out that all loop functions $J_{\pi, R}^{(\pi)}\left(p, z_{0}\right), J_{R}^{(\pi)}\left(p, z_{0}\right) \sim Q^{4}$ and $J_{V, R}^{(\pi)}\left(p, z_{0}\right) \sim$ $Q^{6}$ contribute to the renormalized inverse coupling $g_{R}^{-1}$ at chiral order $Q^{4}$ : we discover here the presence of the enhancement factor $g_{R}^{-1} \sim Q^{-2}$ which implies that at leading chiral order pion production close to threshold is driven by the pion production vertex (124):

$$
\begin{aligned}
\Im \delta_{\left[{ }^{1} S_{0}\right]}(p) & \simeq \frac{g_{A} \zeta_{\pi} m_{\pi}^{4}}{4 \pi f_{\pi}} \frac{\left(p^{2}-\mu_{\sigma}^{2}\right)\left(p^{2}-\mu_{\pi}^{2}\right)^{2}}{4 \pi^{2} \zeta_{\sigma}^{2} \mu_{\sigma}^{4} p m} \Theta\left(p-\mu_{\pi}\right) \\
& +2 \frac{g_{A}^{2} m_{\pi}^{4}}{\left(4 \pi f_{\pi}\right)^{2}} \frac{\left(p^{2}-\mu_{\sigma}^{2}\right)^{2}\left(p^{2}-\mu_{\pi}^{2}\right)^{2}}{\zeta_{\sigma}^{2} \mu_{\sigma}^{4} p^{3} m} \Theta\left(p-\mu_{\pi}\right)
\end{aligned}
$$

with:

$$
\lambda^{4} g^{(\pi)} g_{R}^{-1}=\frac{m}{2 \pi} \frac{\zeta_{\pi}}{p^{2}-\mu_{\sigma}^{2}}+\cdots
$$

In (130) we dropped some overall normalization induced by pionic vertex corrections for simplicity. Our result clearly demonstrates a subtle interplay of the pion production scale $\mu_{\pi}$ and $\mu_{\sigma}$. Close to the chiral limit with $\mu_{\pi} \ll \mu_{\sigma}$ the second term in (130) is dominant for the threshold region with $p \simeq \mu_{\pi}$. For a realistic pion mass with $\mu_{\pi} \simeq \mu_{\sigma}$ we find the first term in (130) most important since it is suppressed by only one power in $p^{2}-\mu_{\sigma}^{2}$ as compared to the two powers of the second term.

In Fig. 5 we confront (130) with Arndt's SP99 analysis [33]. The inelasticity parameter $\rho$ with $\Im \delta=-\ln \cos \rho$ as well as the real part of the phase shift are described well with $\xi_{0}^{(2)}=0.751 \mathrm{fm}, \xi_{2}^{(0)}=0.222 \mathrm{fm}, \xi_{\sigma}=-783.5 \mathrm{MeV}$, $\mu_{\sigma}=350.4 \mathrm{MeV}, z_{0} \simeq-35.27 \mathrm{MeV}$, and $\zeta_{\pi}=0.301 \mathrm{fm}$. Note that the set of parameters is rather close to the values given in Tab. 1 indicating once again the stability of our expansion. The pion production parameter $\zeta_{\pi}$ turns out natural since it implies $g^{(\pi)} \simeq-0.02$. Fig. 5 shows also results of the perturbative expression (130) with $\zeta_{\pi}=0.301 \mathrm{fm}$ (dashed line) and $\zeta_{\pi}=0 \mathrm{fm}$ (dashed-dotted line). This demonstrates that pion production is driven by the vertex (124) and furthermore that the production turns non-perturbative for momenta $p>400 \mathrm{MeV}$.

The pion production cross section was studied close to threshold by a series 

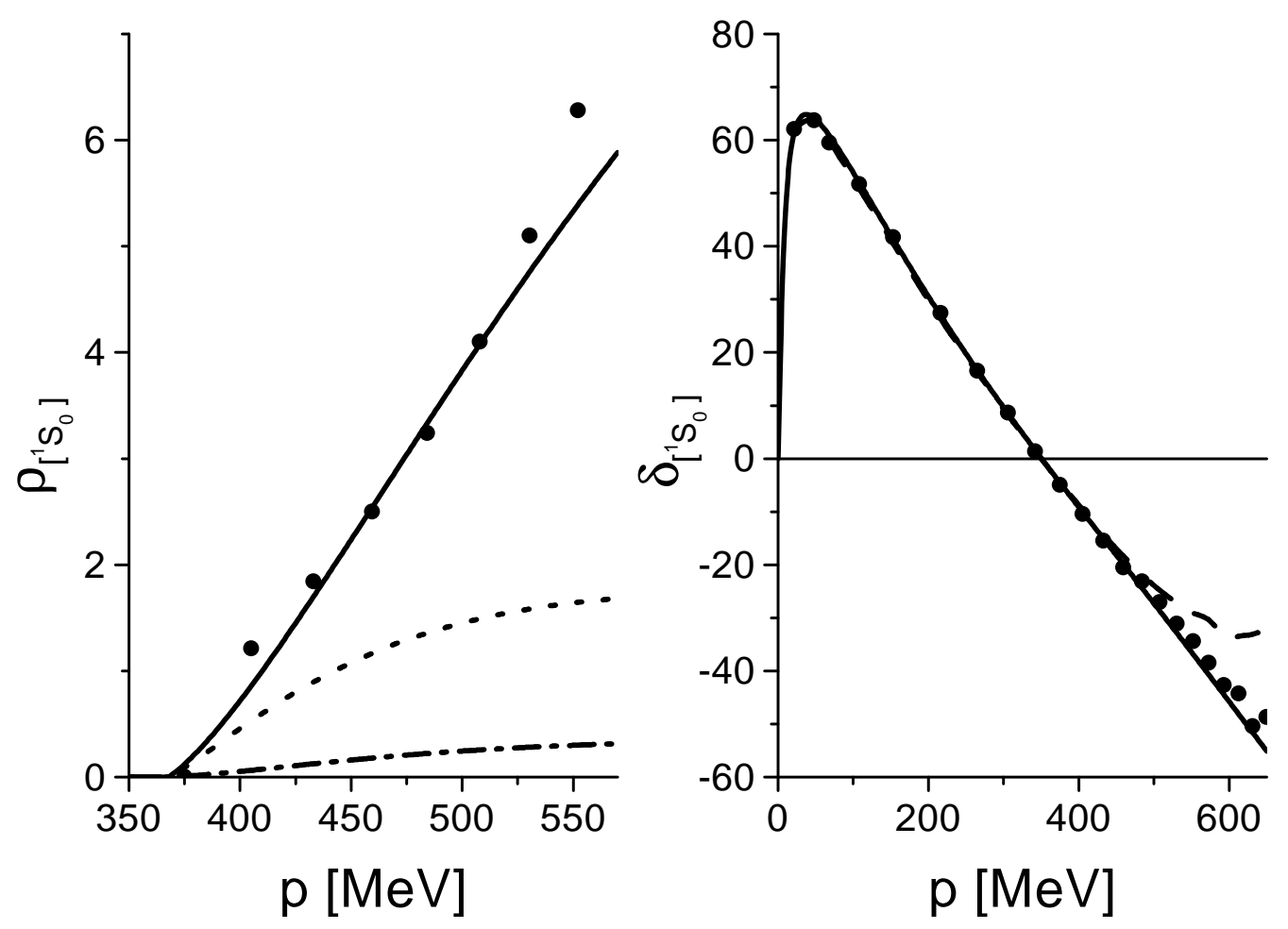

Fig. 5. The left figure shows the inelasticity parameter $\rho$ with $\Im \delta=-\ln \cos \rho$ and the right figure the real part of the ${ }^{1} S_{0}$-phase shift. Our partial UCE-Q ${ }^{2}$ calculation as described in the text (129) is given by the solid line. The dashed line is the result of the Bonn potential and the dots represent the SP99 analysis [33]. The dotted and dash-dotted lines follow from the perturbative expression (130) with $\zeta_{\pi}=0.301 \mathrm{fm}$ and $\zeta_{\pi}=0 \mathrm{fm}$ respectively.

of recent works [39-44] in the context of chiral perturbation theory. Since the total pion production cross section

$$
\sigma_{\pi-\text { production }}=\frac{\pi}{2 p^{2}} \sum_{l J}(2 J+1)\left(1-\exp \left(-4 \Im \delta_{l J}\right)\right)
$$

receives contributions also from higher partial waves even close to threshold [45], the comparison is not directly possible at this stage. Nevertheless it is amusing to observe that our production mechanism contradicts conventional chiral attempts to describe pion production insofar that in our scheme the production vertex (124) contributes at leading rather than at subleading order [39-44]. We therewith confirm phenomenological studies which demonstrate the importance of the $\sigma$-meson exchange $[46,47]$. For a review of one-pion production in nucleon-nucleon collisions see [48].

We find our result for the ${ }^{1} S_{0}$ partial wave encouraging and therefore the unitary chiral expansion scheme should be applied also to the spin triplet swave and higher partial waves. In particular we expect that the zero of the ${ }^{3} S_{1}$ 
and ${ }^{3} P_{0}$-phase at $E_{\text {lab }} \simeq 300 \mathrm{MeV}$ and $E_{\text {lab }} \simeq 200 \mathrm{MeV}$ respectively is driven by a mechanism analogous to the mechanism presented for the ${ }^{1} S_{0}$ phase.

\section{Acknowledgments}

The author acknowledges useful discussions with Bengt Friman and Evgueni Kolomeitsev.

\section{A Partial wave representation of invariant amplitudes}

The on-shell nucleon-nucleon scattering amplitude is given in terms of five isospin zero amplitudes $a_{i}^{(0)}(t, u)$ and five isospin one amplitudes $a_{i}^{(1)}(u, t)$ as follows:

$$
T_{c}(u, t)=\mathcal{P}_{0} \sum_{i=0}^{5} a_{i}^{(0)}(u, t) T_{i}+\mathcal{P}_{1} \sum_{i=0}^{5} a_{i}^{(1)}(u, t) T_{i}
$$

with the Dirac tensors

$$
\begin{aligned}
& T_{1}=\left(\gamma_{5} C^{-1} \times C \gamma_{5}\right), \\
& T_{2}=\left(\gamma_{\alpha} C^{-1} \times C \gamma^{\alpha}\right), \\
& T_{3}=\left(C^{-1} \times C\right) \\
& T_{4}=\left(\gamma_{\alpha} C^{-1} \times C \gamma^{\alpha}\right)-\frac{1}{2}\left(\sigma_{\alpha \beta} C^{-1} \times C \sigma^{\alpha \beta}\right), \\
& T_{5}=\left(\gamma_{5} C^{-1} \times C \gamma_{5}\right)+\left(\gamma_{5} \gamma_{\alpha} C^{-1} \times C \gamma_{5} \gamma^{\alpha}\right)
\end{aligned}
$$

and the isospin projection operators:

$$
\begin{aligned}
& \mathcal{P}_{0}=\frac{1}{2}\left(\tau_{2} \times \tau_{2}\right), \\
& \mathcal{P}_{1}=\frac{1}{2}\left(\vec{\tau} \tau_{2} \times \tau_{2} \vec{\tau}\right)
\end{aligned}
$$

The tensor product ' $x^{\prime}$ in (A.2) is defined via the replacement rule

$$
\bar{\Gamma} C^{-1} \times C \Gamma \rightarrow\left(\bar{u}\left(p_{1}^{\prime}\right) \bar{\Gamma} C^{-1} \bar{u}^{t}\left(p_{2}^{\prime}\right)\right)\left(u^{t}\left(p_{2}\right) C \Gamma u\left(p_{1}\right)\right)
$$

with the incoming momenta $p_{1,2}$ and the outgoing momenta $p_{1,2}^{\prime}$. The invariant amplitudes $a_{i}^{(I)}(u, t)$ can be uniquely reconstructed from the nucleon phase 
shifts [35]. It is convenient first to express the amplitudes in terms of auxiliary amplitudes $f_{i}$ which have a more transparent expansion in partial wave amplitudes:

$$
\begin{aligned}
& a_{1}=2 \frac{m^{2}}{s} f_{1}-\frac{m^{2}}{s} f_{3}-\frac{m^{2}}{s} \frac{u-t}{u+t} f_{4}-\frac{m}{\sqrt{s}} \frac{u-t}{u+t} f_{5}, \\
& a_{2}=-\frac{m}{\sqrt{s}} f_{5}, \\
& a_{3}=2 \frac{m^{2}}{u+t} f_{2}+m^{2} \frac{u-t}{(u+t)^{2}} f_{4}+\frac{m}{\sqrt{s}} \frac{u-t}{(u+t)^{2}}\left(s+4 m^{2}\right) f_{5}, \\
& a_{4}=-\frac{m^{2}}{u+t} f_{4}-\frac{m \sqrt{s}}{u+t} f_{5}, \\
& a_{5}=-\frac{m^{2}}{u+t} f_{3} .
\end{aligned}
$$

For the reader's convenience we also provide the inverse relations

$$
\begin{aligned}
& f_{1}=\frac{s}{2 m^{2}} a_{1}-\frac{u-t}{2 m^{2}} a_{4}-\frac{u+t}{2 m^{2}} a_{5} \\
& f_{2}=2 \frac{u-t}{u+t} a_{2}+\frac{u+t}{2 m^{2}} a_{3}-\frac{u-t}{2 m^{2}} a_{4}, \\
& f_{3}=-\frac{u+t}{m^{2}} a_{5} \\
& f_{4}=\frac{s}{m^{2}} a_{2}-\frac{u+t}{m^{2}} a_{4} \\
& f_{5}=-\frac{\sqrt{s}}{m} a_{2} .
\end{aligned}
$$

The amplitudes $f_{i}$ are now easily expressed in terms of coupled $f_{11}^{(j)}, f_{22}^{(j)}, f_{12}^{(j)}$ and uncoupled $f_{1}^{(j)}, f_{0}^{(j)}$ partial wave amplitudes

$$
\begin{aligned}
f_{1} & =\sum_{j=0}^{\infty}(2 j+1) f_{0}^{(j)} d_{00}^{(j)}(\theta), \\
f_{2} & =\sum_{j=0}^{\infty}(2 j+1) f_{11}^{(j)} d_{00}^{(j)}(\theta), \\
f_{3} & =(t+u) \sum_{j=0}^{\infty}(2 j+1) f_{1}^{(j)}\left(\frac{d_{11}^{(j)}(\theta)}{2 u}+\frac{d_{-11}^{(j)}(\theta)}{2 t}\right) \\
& +(t+u) \sum_{j=0}^{\infty}(2 j+1) f_{22}^{(j)}\left(\frac{d_{11}^{(j)}(\theta)}{2 u}-\frac{d_{-11}^{(j)}(\theta)}{2 t}\right),
\end{aligned}
$$




$$
\begin{aligned}
f_{4} & =(t+u) \sum_{j=0}^{\infty}(2 j+1) f_{1}^{(j)}\left(\frac{d_{11}^{(j)}(\theta)}{2 u}-\frac{d_{-11}^{(j)}(\theta)}{2 t}\right) \\
& +(t+u) \sum_{j=0}^{\infty}(2 j+1) f_{22}^{(j)}\left(\frac{d_{11}^{(j)}(\theta)}{2 u}+\frac{d_{-11}^{(j)}(\theta)}{2 t}\right), \\
f_{5} & =-(t+u) \sum_{j=0}^{\infty}(2 j+1) f_{12}^{(j)} \frac{d_{10}^{(j)}(\theta)}{2 \sqrt{u t}}
\end{aligned}
$$

where $d_{m^{\prime} m}^{(j)}(\theta)=\left\langle j m^{\prime}\left|e^{-i \theta J_{y}}\right| j m\right\rangle$. The sum over the total angular momentum $j$, in (A.7) is constrained by proper selection rules. In (A.7) we restricted the c.m. scattering angle $0<\theta<\pi$ for technical convenience.

The coupled amplitudes $f_{11}^{(j)}, f_{22}^{(j)}$ and $f_{12}^{(j)}$ are expressed in terms of the amplitudes $f_{l=j+1}, f_{l=j-1}$ with well defined angular momentum $l$, and the transition amplitude $f_{ \pm}$as follows:

$$
\begin{aligned}
& f_{11}^{(j)}=\frac{1+j}{2 j+1} f_{l=j+1}+\frac{j}{2 j+1} f_{l=j-1}+2 \frac{\sqrt{j(j+1)}}{2 j+1} f_{ \pm}, \\
& f_{22}^{(j)}=\frac{j}{2 j+1} f_{l=j+1}+\frac{1+j}{2 j+1} f_{l=j-1}-2 \frac{\sqrt{j(j+1)}}{2 j+1} f_{ \pm}, \\
& f_{12}^{(j)}=-\frac{\sqrt{j(j+1)}}{2 j+1} f_{l=j+1}+\frac{\sqrt{j(j+1)}}{2 j+1} f_{l=j-1}+\frac{1}{2 j+1} f_{ \pm} .
\end{aligned}
$$

The partial wave amplitudes are given in terms of the phase shifts. For the uncoupled amplitudes $f_{0}^{(j)}$ and $f_{1}^{(j)}$ one defines

$$
f^{(j)}=\frac{4 \pi}{m^{2}} \sqrt{\frac{-s}{u+t}} \frac{1}{i}\left(e^{2 i \delta_{j}}-1\right)
$$

with the partial wave phase shift $\delta_{j}$. The coupled partial wave amplitudes

$$
\begin{aligned}
f_{l=j \pm 1} & =\frac{4 \pi}{m^{2}} \sqrt{\frac{-s}{u+t}} \frac{1}{i}\left(\cos \left(2 \epsilon_{j}^{S}\right) e^{2 i \delta_{l=j \pm 1}^{S}}-1\right) \\
f_{j}^{ \pm} & =\frac{4 \pi}{m^{2}} \sqrt{\frac{-s}{u+t}} \sin \left(2 \epsilon_{j}^{S}\right) e^{i\left(\delta_{l=j-1}^{S}+\delta_{l=j+1}^{S}\right)}
\end{aligned}
$$

are expressed in terms of the phase shift $\delta_{l=j \pm 1}^{S}$ and the mixing angle $\epsilon_{j}$ according to Stapp's convention [49]. Note that the spin singlet partial wave phase shift $\delta\left({ }^{1} S_{0}\right)$ sits in $f_{1}$. 


\section{B Evaluation of loops}

In this appendix we derive analytic results for the one, two and three-loop function needed for the nucleon scattering amplitude at leading chiral orders. Since we apply a relativistic version of the chiral Lagrangian the non-relativistic expansion in $1 / m$ is performed at the level of the loop integrals. Technically this can be achieved by expanding the spectral density in powers of $1 / \mathrm{m}$ and then recovering the real part via a dispersion relation. Equivalently one may perform the energy integrations in the loop function first and then expand the integrand before working out the remaining three momentum integrals. When dealing with fermions a further complication arises due to the spin structure of fermions. However, any diagram involving complicated Dirac structure can be expressed in terms of scalar master loop integrals which naturally arise in scalar quantum field theory. This reduction can be achieved purely algebraically relying only on covariance and the shift invariance of the integration

measure $d^{4} l$ (as implied for example by dimensional regularization). We therefore exemplify our techniques first at hand of these master loop functions. Consider the scalar functions:

$$
\begin{aligned}
I_{2, N}(s) & =-i \int \frac{d^{4} l}{(2 \pi)^{4}} S_{N}(P-l) S_{N}(K+l) \\
I_{3, N}(s) & =i \int \frac{d^{4} l}{(2 \pi)^{4}} S_{\pi}\left(l+\frac{1}{2} Q\right) S_{N}(P-l) S_{N}(K+l) \\
I_{4, N}(s, t) & =-i \int \frac{d^{4} l}{(2 \pi)^{4}} S_{\pi}\left(l+\frac{1}{2} Q\right) S_{\pi}\left(l-\frac{1}{2} Q\right) S_{N}(P-l) S_{N}(K+l)(
\end{aligned}
$$

with $S_{i}(p)=1 /\left(p^{2}-m_{i}^{2}+i \epsilon\right)$. For technical convenience we introduce the four vectors $P, K$ and $Q$ as follows:

$$
\begin{array}{ll}
p_{1}=K+\frac{1}{2} Q, & p_{1}^{\prime}=K-\frac{1}{2} Q, \\
p_{2}=P-\frac{1}{2} Q, & p_{2}^{\prime}=P+\frac{1}{2} Q .
\end{array}
$$

The master functions are easily calculated by means of dispersion techniques:

$$
\begin{aligned}
I_{2, N}(s) & =\int_{4 m^{2}}^{4\left(m^{2}+\Lambda^{2}\right)} \frac{d s^{\prime}}{\pi} \frac{\rho_{2, N}\left(s^{\prime}\right)}{s^{\prime}-s}, \quad I_{3, N}(s)=\int_{4 m^{2}}^{4\left(m^{2}+\Lambda^{2}\right)} \frac{d s^{\prime}}{\pi} \frac{\rho_{3, N}\left(s^{\prime}\right)}{s^{\prime}-s} \\
I_{4, N}(s, t) & =\int_{4 m^{2}}^{4\left(m^{2}+\Lambda^{2}\right)} \frac{d s^{\prime}}{\pi} \frac{\rho_{4, N}\left(s^{\prime}, t\right)}{s^{\prime}-s}
\end{aligned}
$$


with $(P+K)^{2}=s$. The finite cutoff $\Lambda<m$ is kept at this intermediate stage in order to mathematically justify the $1 / m$ expansion and provide an instructive guide how to define a suitable regularization scheme. The cutoff $\Lambda$ will ultimately be removed and may be replaced by an appropriate scale arising from dimensional regularization. We derive the s-channel spectral densities:

$$
\begin{aligned}
\rho_{2, N}(s) & =\frac{1}{8 \pi} \sqrt{\frac{1}{4}-\frac{m^{2}}{s}}, \\
\rho_{3, N}(s) & =\frac{1}{16 \pi} \frac{1}{\sqrt{4 m^{2}-u-t}} \frac{1}{\sqrt{-u-t}} \ln \left(\frac{m_{\pi}^{2}-u-t}{m_{\pi}^{2}}\right), \\
\rho_{4, N}(s, t) & =\frac{1}{4 \pi} \frac{1}{\sqrt{-t}} \frac{1}{\sqrt{4 m^{2}-u-t}} \frac{1}{\sqrt{b(s, t)}} \operatorname{arctanh} \sqrt{\frac{t(u+t)}{b(s, t)}}
\end{aligned}
$$

with $u+t=4 m^{2}-s$ and

$$
b(s, t)=4 m_{\pi}^{4}-4 m_{\pi}^{2}(u+t)+t(u+t) .
$$

In the algebraic reduction of the pion nucleon box diagram we will encounter further master loop functions:

$$
\begin{aligned}
& I_{2}\left(p^{2}\right)=-i \int \frac{d^{4} l}{(2 \pi)^{4}} S_{N}(l+p) S_{\pi}(l) \\
& I_{2, \pi}(t)=i \int \frac{d^{4} l}{(2 \pi)^{4}} S_{\pi}\left(l+\frac{1}{2} Q\right) S_{\pi}\left(l-\frac{1}{2} Q\right) \\
& I_{3, \pi}(t)=i \int \frac{d^{4} l}{(2 \pi)^{4}} S_{\pi}\left(l+\frac{1}{2} Q\right) S_{\pi}\left(l-\frac{1}{2} Q\right) S_{N}(l+K) .
\end{aligned}
$$

We point out that the master loops of (B.6) are not independent of the master loops introduced in (B.1). They are interrelated by exact algebraic identities required for the cancellation of kinematical singularities:

$$
\begin{aligned}
I_{2}\left(m^{2}\right) & =I_{2, N}\left(4 m^{2}\right)-m_{\pi}^{2} I_{3, N}\left(4 m^{2}\right) \\
I_{3, \pi}(t)= & I_{3, N}\left(4 m^{2}-t\right)-\left(m_{\pi}^{2}-\frac{1}{2} t\right) I_{4, N}\left(4 m^{2}-t, t\right) \\
\left.\left(2 m_{\pi}^{2}-t\right)^{2} \frac{\partial}{\partial s} I_{4, N}(s, t)\right|_{s=4 m^{2}-t}=I_{3, N}\left(4 m^{2}-t\right) & \\
& -\frac{1}{2}\left(4 m_{\pi}^{2}-t\right) I_{4, N}\left(4 m^{2}-t, t\right)+2\left(2 m_{\pi}^{2}-t\right) I_{3, N}^{\prime}\left(4 m^{2}-t\right) \\
& -\frac{2}{t}\left(m_{\pi}^{2} I_{3, N}\left(4 m^{2}-t\right)-I_{2, N}\left(4 m^{2}-t\right)+I_{2}\left(m^{2}\right)\right) \\
& -\frac{2}{4 m^{2}-t}\left(\left(m_{\pi}^{2}-\frac{1}{2} t\right) I_{3, \pi}(t)+I_{2, \pi}(t)-I_{2}\left(m^{2}\right)\right) .
\end{aligned}
$$


The identities (B.7) can be verified for example in dimensional regularization ${ }^{8}$. Any consistent regularization procedure must respect (B.7) order by order in the $1 / m$-expansion. Once we specify our regularization for $I_{i, N}(s)$ in terms of the cutoff $\Lambda$ the regularized loops $I_{2}\left(p^{2}\right), I_{2, \pi}(t)$ and $I_{3, \pi}(t)$ must be compatible with (B.7).

One may conjecture that the triangle loops $I_{2, \pi}(t)$ and $I_{3, \pi}(t)$ could be evaluated by means of a dispersion integral:

$$
I_{2, \pi}(t)=\int_{4 m_{\pi}^{2}}^{4\left(m_{\pi}^{2}+\bar{\Lambda}^{2}\right)} \frac{d t^{\prime}}{\pi} \frac{\rho_{2, \pi}\left(t^{\prime}\right)}{t^{\prime}-t}, \quad I_{3, \pi}(t)=\int_{4 m_{\pi}^{2}}^{4\left(m_{\pi}^{2}+\bar{\Lambda}^{2}\right)} \frac{d t^{\prime}}{\pi} \frac{\rho_{3, \pi}\left(t^{\prime}\right)}{t^{\prime}-t}
$$

with the t-channel spectral densities:

$$
\begin{aligned}
& \rho_{2, \pi}(t)=\frac{\Theta\left(t-4 m_{\pi}^{2}\right)}{8 \pi} \sqrt{\frac{1}{4}-\frac{m_{\pi}^{2}}{t}} \\
& \rho_{3, \pi}(t)=\frac{1}{8 \pi} \frac{\Theta\left(t-4 m_{\pi}^{2}\right)}{\sqrt{t\left(4 m^{2}-t\right)}} \arctan \left(\sqrt{4 m^{2}-t} \frac{\sqrt{t-4 m_{\pi}^{2}}}{t-2 m_{\pi}^{2}}\right)
\end{aligned}
$$

for $t>4 m_{\pi}^{2}$. The pion bubble $I_{2, \pi}(t)$ is rendered finite by one subtraction. Performing the dispersion integral for $I_{2, \pi}(t)$ in (B.8) one finds:

$$
\begin{aligned}
I_{2, \pi}\left(4 m_{\pi}^{2}\right) & =\frac{1}{8 \pi^{2}} \operatorname{arcsinh}\left(\frac{\bar{\Lambda}}{m_{\pi}}\right) \\
I_{2, \pi}(t) & =I_{2, \pi}(0)-\frac{1}{8 \pi^{2}}\left(\sqrt{\frac{4 m_{\pi}^{2}-t}{t}} \arctan \sqrt{\frac{t}{4 m_{\pi}^{2}-t}}-1\right)
\end{aligned}
$$

where we applied the large $\bar{\Lambda}$-cutoff limit in the subtracted loop. We point out, however, that the cutoff $\bar{\Lambda}$ in $I_{2, \pi}(t)$ and $I_{3, \pi}(t)$ must not be identified with $\Lambda$. The $1 / m$ expansion of the loop integral $I_{3, \pi}(t)$ is subtle. The regularization of $I_{3, \pi}(t)$ by means of the cutoff $\bar{\Lambda} \ll m$ is incompatible with the $1 / m$ expansion. This follows from the fact that the $1 / m$ expansion of the t-channel spectral density leads, at subleading orders, to singularities in the dispersion integral at $t^{\prime}=4 m_{\pi}^{2}$ whereas $I_{3, \pi}(t)$ given via (B.7) leads to well defined moments in the $1 / m$ expansion.

8 More conveniently the identities (B.7) can be derived algebraically if one requires the reduced loop function $b_{1,3}(s, t)$ of the pion-nucleon box diagram not to be singular at $u=0$ and $u+t=0$. See appendix B.6. 
At the one-loop level we complete the collection of master loops with the pionic and nucleonic tadpole diagrams $I_{1, \pi}$ and $I_{1, N}$ :

$$
\begin{aligned}
I_{1, N} & =i \int \frac{d^{4} l}{(2 \pi)^{4}} \frac{1}{l^{2}-m_{N}^{2}+i \epsilon}=\int_{4 m^{2}}^{4\left(m^{2}+\Lambda^{2}\right)} \frac{d s}{2 \pi} \Im I_{2, N}(s) \\
& =\frac{1}{12 \pi^{2}} \frac{\Lambda^{3}}{m}\left(1+\mathcal{O}\left(\frac{\Lambda^{2}}{m^{2}}\right)\right) \\
I_{1, \pi} & =i \int \frac{d^{4} l}{(2 \pi)^{4}} \frac{1}{l^{2}-m_{\pi}^{2}+i \epsilon}=\int_{4 m_{\pi}^{2}}^{4\left(m_{\pi}^{2}+\bar{\Lambda}^{2}\right)} \frac{d t}{2 \pi} \Im I_{2, \pi}(t) \\
& =\frac{1}{8 \pi^{2}}\left(\bar{\Lambda} \sqrt{m_{\pi}^{2}+\bar{\Lambda}^{2}}-m_{\pi}^{2} \operatorname{arcsinh}\left(\frac{\bar{\Lambda}}{m_{\pi}}\right)\right)
\end{aligned}
$$

where consistency of the cutoff regularization relates $I_{1, N}$ with $\Im I_{2, N}(s)$ and $I_{1, \pi}$ with $\Im I_{2, \pi}(t)$. It is crucial to observe that the proper $\Lambda$ dependence of the pionic tadpole $I_{1, \pi}$ follows from a further exact algebraic identity

$$
\begin{aligned}
I_{1, \pi}-I_{1, N} & =6 m^{2} m_{\pi}^{2}\left(m_{\pi}^{2} \frac{d}{d s} I_{3, N}\left(4 m^{2}\right)-\frac{d}{d s} I_{2, N}\left(4 m^{2}\right)\right) \\
& +\left(2 m^{2} m_{\pi}^{2}+m_{\pi}^{4}\right) I_{3, N}\left(4 m^{2}\right)+\left(m^{2}-m_{\pi}^{2}\right) I_{2, N}\left(4 m^{2}\right)
\end{aligned}
$$

which may be verified in dimensional regularization. Again we insist that (B.12) must be respected by the $1 / m$-expansion. In particular (B.12) implies a non-trivial matching of the s-channel cutoff parameters $\Lambda$ and the t-channel cutoff $\bar{\Lambda}$.

We point out that all $t$-channel loops $I_{3, \pi}(t), I_{2, \pi}(t)$ and $I_{1, \pi}$ can in fact be defined via (B.7) and (B.12) in terms of the s-channel loops $I_{4, N}(s, t), I_{3, N}(s)$ and $I_{2, N}(s)$. It is, however, convenient to simplify our regularization scheme and derive more explicit expressions for the loop functions in order to facilitate the renormalization procedure. First we assume that the diagrams are already partly $1 / m$ renormalized via the construction (46). This implies that the factor $1 / \sqrt{s^{\prime}}$ in the spectral functions $\rho_{i, N}\left(s^{\prime}\right)$ can be pulled out of the dispersion integral (B.3). This procedure moves a large class of power divergencies of the form $(\Lambda / m)^{n}$ into the interaction kernel where they are absorbed into the local interaction terms. Next we systematically drop nucleonic tadpole diagrams since they also involve multiple powers of the cutoff $\Lambda^{n}$ with $n \geq 3$. Such terms are ill-defined and are ultimately canceled from contributions induced by the properly constructed cutoff dependence of the path-integral measure [27]. Finally we apply the large cutoff limit for all finite parts. Our regularization scheme leads now to at most linear divergent one-loop functions. 
In more formal terms our regularization scheme is defined by writing the oneloop functions $I_{2, N}(s), I_{3, N}(s)$ and $I_{4, N}(s, t)$ as follows:

$$
\begin{aligned}
I_{2, N}(s) & =\frac{1}{8 \pi \sqrt{s}}\left(\frac{2}{\pi} \Lambda+i p\right)+\Delta I_{2, N}(s) \\
I_{3, N}(s) & =\frac{1}{16 \pi \sqrt{s}} \frac{i}{p} \ln \left(1-2 i \frac{p}{m_{\pi}}\right)+\Delta I_{3, N}(s), \\
I_{4, N}(s, t) & =\frac{1}{4 \pi \sqrt{s}} \frac{1}{\sqrt{-t b(s, t)}}\left(i \operatorname{arctanh}\left(\sqrt{\frac{t(u+t)}{b(s, t)}}\right)\right. \\
& +\frac{1}{2} \arctan \left(\frac{\sqrt{-t b(s, t)}}{4 m_{\pi}^{3}}-\frac{\sqrt{-u-t}}{m_{\pi}}\left(1-\frac{t}{4 m_{\pi}^{2}}\right)\right) \\
& \left.+\frac{1}{2} \arctan \left(\frac{\sqrt{-t b(s, t)}}{4 m_{\pi}^{3}}+\frac{\sqrt{-u-t}}{m_{\pi}}\left(1-\frac{t}{4 m_{\pi}^{2}}\right)\right)\right) \\
& +\Delta I_{4, N}(s, t) .
\end{aligned}
$$

where

$$
\begin{gathered}
\Delta I_{2, N}(s)=-\int_{4 m^{2}}^{4\left(m^{2}+\Lambda^{2}\right)} \frac{d s^{\prime}}{\pi} \frac{\Im I_{2, N}\left(s^{\prime}\right)}{s+\sqrt{s s^{\prime}}}, \\
\Delta I_{3, N}(s)=-\int_{4 m^{2}}^{4\left(m^{2}+\Lambda^{2}\right)} \frac{d s^{\prime}}{\pi} \frac{\Im I_{3, N}\left(s^{\prime}\right)}{s+\sqrt{s s^{\prime}}}, \\
\Delta I_{4, N}(s, t)=-\int_{4 m^{2}}^{4\left(m^{2}+\Lambda^{2}\right)} \frac{d s^{\prime}}{\pi} \frac{\Im I_{4, N}\left(s^{\prime}, t\right)}{s+\sqrt{s^{\prime} s}} .
\end{gathered}
$$

Note that for example the separation of the term $\Delta I_{2, N}(s)$ from $I_{2, N}(s)$ in (B.13) is induced uniquely by our construction $(45,46)$. The divergent structure $\Delta I_{2, N}(s)$ can systematically be absorbed into local counter terms in accordance with (46). This follows from the representation in (B.14) which shows that $\Delta I_{2, N}(s)$ can be expanded in a Taylor series around $s=4 \mathrm{~m}^{2}$. In our simplified regularization scheme such terms are from now on simply dropped. The loop functions $I_{3, N}(s)$ and $I_{4, N}(s, t)$ require special attention since $\Delta I_{3, N}(s)$ leads to chiral log terms and $\Delta I_{4, N}(s, t)$ depends on $t$ in a non-polynomial fashion. It is instructive to consider first $\Delta I_{3, N}(s)$ and $\Delta I_{4, N}(s, t)$ as defined via (B.14) in terms of the finite cutoff $\Lambda$ and inspect the point $s=4 m^{2}, t=0$ :

$$
\Delta I_{3, N}\left(4 m^{2}\right)=\frac{\Lambda}{64 \pi^{2} m^{3}}\left(1-\ln \left(\frac{2 \Lambda}{m_{\pi}}\right)-\frac{\pi}{2} \frac{m_{\pi}}{\Lambda}+\mathcal{O}\left(\frac{\Lambda^{2}}{m^{2}}\right)\right)
$$




$$
\Delta I_{4, N}\left(4 m^{2}, 0\right)=-\frac{\Lambda}{64 \pi^{2} m^{3} m_{\pi}^{2}}\left(1+\mathcal{O}\left(\frac{\Lambda^{2}}{m^{2}}\right)\right)
$$

where $\Lambda<m$. Note that $\Delta I_{3, N}\left(4 m^{2}\right)$ must not be considered as a counter term since it is non-analytic in the squared pion mass. Also the term $\Lambda / m_{\pi}^{2}$ cannot be absorbed into local interaction terms of the chiral Lagrangian. The result (B.15) signals an infrared singularity induced by the $1 / m$ expansion and asks for a more careful regularization procedure. In fact the solution to this puzzle follows simply if one evaluates $\Delta I_{4, N}\left(4 m^{2}, 0\right)$ for an infinite cutoff $\Lambda>m$ :

$$
\begin{aligned}
\Delta I_{3, N}\left(4 m^{2}\right) & =\frac{-1}{16 \pi^{2} m^{2}}\left(\ln \left|\frac{m}{m_{\pi}}\right|+\pi \frac{m}{m_{\pi}}\right. \\
& \left.-\sqrt{\frac{4 m^{2}}{m_{\pi}^{2}}-1} \arctan \left(\sqrt{\frac{4 m^{2}}{m_{\pi}^{2}}-1}\right)\right) \\
& =\frac{-1}{16 \pi^{2} m^{2}}\left(1+\ln \left|\frac{m}{m_{\pi}}\right|+\frac{\pi}{8} \frac{m_{\pi}}{m}-\frac{1}{12} \frac{m_{\pi}^{2}}{m^{2}}+\mathcal{O}\left(\frac{m_{\pi}^{3}}{m^{3}}\right)\right) . \\
\Delta I_{4, N}\left(4 m^{2}, 0\right) & =\frac{-1}{16 \pi^{2} m m_{\pi}^{3}}\left(\frac{\pi}{2}-\frac{2 m}{\sqrt{4 m^{2}-m_{\pi}^{2}}} \arctan \left(\sqrt{\frac{4 m^{2}}{m_{\pi}^{2}}}-1\right)\right) \\
& \left.=\frac{-1}{32 \pi^{2} m^{2} m_{\pi}^{2}}\left(1-\frac{\pi}{8} \frac{m_{\pi}}{m}+\frac{1}{6} \frac{m_{\pi}^{2}}{m^{2}}+\mathcal{O}\left(\frac{m_{\pi}^{3}}{m^{3}}\right)\right) . \quad \text { B. } 16\right)
\end{aligned}
$$

This is a surprising result since it demonstrates that the chiral $\log$ term $\ln \left(m_{\pi}\right)$ in (B.16) results in part from modes probing large nucleon virtuality with $\Lambda>m$.

In order to perform the $1 / m$ expansion of $\Delta I_{4, N}(s, t)$ and derive explicit results it is convenient to perform the angle average in (8) after the $s^{\prime}$-integration in (B.14). Now with $\Lambda=\infty$ in (B.14) we obtain:

$$
\begin{aligned}
\Delta I_{4, N}\left(4 m^{2}, t\right) & =\frac{-1}{8 \pi^{2}} \int_{-1 / 2}^{1 / 2} \frac{d x}{m_{\pi}^{4}}\left(\frac{\pi}{2 \sqrt{a(x, t)}}-\frac{\arctan (\sqrt{-1+a(x, t)})}{\sqrt{-1+a(x, t)}}\right) \\
a(x, t) & =\frac{m^{2}}{m_{\pi}^{2}}\left(4+\frac{t}{m_{\pi}^{2}}\left(4 x^{2}-1\right)\right)
\end{aligned}
$$

The $1 / m$ expansion of $\Delta I_{4, N}(s, t)$ follows upon expanding the integrand of (B.17) in powers of $1 / a$ : 


$$
\left.\Delta I_{4, N}(s, t)=\frac{-1}{8 \pi^{2} m^{2}}\left(\frac{\operatorname{arctanh} \sqrt{\frac{-t}{4 m_{\pi}^{2}-t}}}{\sqrt{4 m_{\pi}^{2}-t} \sqrt{-t}}-\frac{\pi m_{\pi}}{8 m} \frac{1}{4 m_{\pi}^{2}-t}\right)+\mathcal{O}\left(\underset{m^{4}}{\text { ( })}\right) \text { 8, }\right)
$$

where $t<4 m_{\pi}^{2}$. Note that the $1 / m$ expansion of $\Delta I_{4, N}(s, t)$ stops converging at $t=4 m_{\pi}^{2}$.

We turn to the master loop function $I_{3, \pi}(t)$. It is instructive to provide the loop including next-to-leading-order contributions

$$
\begin{aligned}
I_{3, \pi}(t) & =\frac{1}{16 \pi m}\left(1+\frac{t}{8 m^{2}}\right) \frac{1}{\sqrt{-t}} \arctan \left(\frac{\sqrt{-t}}{2 m_{\pi}}\right)-\frac{m_{\pi}}{128 \pi m^{3}} \frac{2 m_{\pi}^{2}-t}{4 m_{\pi}^{2}-t} \\
& +\frac{1}{16 \pi^{2} m^{2}} \frac{2 m_{\pi}^{2}-t}{\sqrt{-t\left(4 m_{\pi}^{2}-t\right)}} \operatorname{arctanh} \sqrt{\frac{-t}{4 m_{\pi}^{2}-t}} \\
& +\Delta I_{3, N}\left(4 m^{2}\right)+\mathcal{O}\left(\frac{1}{m^{4}}\right)
\end{aligned}
$$

which follow with (B.7,B.13,B.18). The result (B.19) is in agreement with the leading expression derived in the heavy-baryon framework [36,37] and with (B.6,B.13). The subleading term of order $1 / \mathrm{m}^{2}$ agrees with a corresponding expression given in [38]. Our result (B.19) confirms that the triangle loop $I_{3, \pi}(t)$ and the scalar pion nucleon box function $I_{4, N}(s, t)$ show a singular branch point at $t=4 m_{\pi}^{2}$ if expanded in $1 / \mathrm{m}$. A systematic resummation leading to a smooth behavior at $t=4 m_{\pi}^{2}$ is proposed in [38].

The function $\Delta I_{4, N}(s, t)$ can alternatively be deduced from (B.7). Imposing the functional form for $I_{2, N}(s), I_{3, N}(s)$ and $I_{4, N}(s, t)$ as given in (B.13) with $\Delta I_{2, N}(s)=0$ we derive:

$$
\Delta I_{4, N}(s, t)=\frac{2 I_{2, \pi}(t)-I_{2}\left(m^{2}\right)+2 m^{2} \Delta I_{3, N}\left(4 m^{2}\right)}{2 m^{2}\left(4 m_{\pi}^{2}-t\right)}+\mathcal{O}\left(\frac{1}{m^{4}}\right)
$$

Our results (B.20) and (B.16) determine the divergent subtraction $I_{2, \pi}(0)$ in terms of $I_{2, N}\left(4 \mathrm{~m}^{2}\right)$. This is a surprising result since it shows that the $1 / \mathrm{m}$ expansion converts the log-divergence in $I_{2, N}(s)$ and $I_{2, \pi}(t)$ into a power divergence. In fact with (B.12) and (B.7) we find that also the pionic tadpole diagram $I_{1, \pi}$ and $I_{2}\left(m^{2}\right)$ must not be dropped and assigned a linear divergence:

$$
\begin{aligned}
I_{1, \pi} & =\left(m^{2}-\frac{1}{4} m_{\pi}^{2}\right) I_{2, N}\left(4 m^{2}\right)+4 m^{2} m_{\pi}^{2} \Delta I_{3, N}\left(4 m^{2}\right)+\mathcal{O}\left(\frac{m_{\pi}^{3}}{m}\right) \\
I_{2, \pi}(0) & =\frac{1}{2} I_{2, N}\left(4 m^{2}\right)-2 m^{2} \Delta I_{3, N}\left(4 m^{2}\right)-\frac{1}{8 \pi^{2}}-\frac{m_{\pi}}{64 \pi m}+\mathcal{O}\left(\frac{m_{\pi}^{2}}{m^{2}}\right)
\end{aligned}
$$




$$
I_{2}\left(m^{2}\right)=I_{2, N}\left(4 m^{2}\right)-m_{\pi}^{2} \Delta I_{3, N}\left(4 m^{2}\right)-\frac{m_{\pi}}{16 \pi m}
$$

We emphasize that our result (B.21) follows from the convenient prescription to systematically drop the divergent terms $I_{1, N}$ and $\Delta I_{2, N}(s)$. This defines our simplified regularization procedure. The regularization of the remaining loop functions is then implied by the algebraic constraints (B.7,B.12). The latter follow for example in dimensional regularization and must be satisfied by any consistent regularization scheme. To this extend it is not surprising to find that for example the pionic tadpole in (B.21) acquires an explicit dependence on the nucleon mass. The only divergent structure left at the one-loop level is $I_{2, N}\left(4 \mathrm{~m}^{2}\right)$. It may be written in terms of the cutoff parameter $\Lambda$ (see (B.13)) or equivalently in terms of a scale resulting from dimensional regularization. Consistent power counting rules arise if we count $I_{2, N}\left(4 m^{2}\right) \sim \Lambda / m \sim Q$. We conclude that the scalar nucleon bubble is in fact the only master loop where we have to strictly insist on $\Lambda<m$ since for $\Lambda \gg m$ one finds $I_{2, N} \sim \ln \Lambda \sim Q^{0}$. All finite loop functions $I_{3, \pi}(t), I_{3, N}(s)$ and $I_{4, N}(s, t)$ must be evaluated with $\Lambda \gg m$ in order to generate the proper chiral log content and avoid infrared singularities (see also [50]).

\section{B.1 Static pion limit}

Here we would like to confront our relativistic scheme with the potential approach. Inherent of the potential approach is the use of the static pion exchange. The pion propagator is expanded

$$
S_{\pi}\left(l_{0}, \vec{l}\right)=\frac{-1}{l^{2}+m_{\pi}^{2}}-\frac{l_{0}^{2}}{\left(l^{2}+m_{\pi}^{2}\right)^{2}}+\mathcal{O}\left(l_{0}^{4}\right)
$$

where it is commonly argued that terms involving the energy transfer $l_{0}^{2}$ are suppressed by $1 / \mathrm{m}$. Mathematically it is not quite obvious that such an expansion is justified and in accordance with covariance since the expansion is highly divergent due to multiple powers of the energy transfer $l_{0}$. We evaluate the master pion nucleon box function $I_{4, N}(u, t)$ in the limit of static pions

$$
I_{4, N}^{(\text {static })}\left(\vec{p}, \vec{p}^{\prime}\right)=\frac{1}{4 m} \int \frac{d^{3} l}{(2 \pi)^{3}} \frac{1}{(\vec{l}+\vec{p})^{2}+m_{\pi}^{2}} \frac{1}{\left(\vec{l}+\vec{p}^{\prime}\right)^{2}+m_{\pi}^{2}} \frac{1}{l^{2}-p^{2}-i \epsilon}
$$

where we chose the center of mass frame with $p^{2}=p^{\prime 2}$ and $\vec{p} \cdot \vec{p}^{\prime}=p^{2} \cos \theta$. Note that we dropped terms suppressed by $1 / \mathrm{m}^{3}$. We compare the static pion result (B.23) with the proper non-relativistic expanded master loop function of (B.13) and find agreement. 


\section{B.2 One-loop bubble}

Start with the one-loop bubble function

$$
\begin{gathered}
J(s)=-i \operatorname{tr} \int \frac{d^{4} l}{(2 \pi)^{4}} C \gamma_{5} S_{F}(P+l) \gamma_{5} C^{-1} S_{F}^{t}(K-l), \\
J(s, \Lambda)=\frac{1}{\pi} \int_{0}^{\Lambda^{2}} \frac{d \bar{p}^{2} \rho_{J}(\bar{p})}{\bar{p}^{2}-p^{2}-i \epsilon}, \quad \rho_{J}(p)=\frac{m p}{2 \pi} \sqrt{1+\frac{p^{2}}{m^{2}}}
\end{gathered}
$$

with the nucleon propagator $S_{F}(p)=1 /(\not p-m+i \epsilon)$. The loop function may be regularized by a cutoff $\Lambda$ introduced in the s-channel dispersion relation

with $s=(P+K)^{2}=4\left(m^{2}+p^{2}\right)$. Equivalently the loop may be expressed in terms of the master functions:

$$
J(s)=2 s I_{2, N}(s)+4 I_{1, N}
$$

The expressions (B.23) and (B.24) agree if the cutoff regularized tadpole is given by (B.11). Our simplified regularization procedure leads to a linear divergent one-loop function $J(s, \Lambda)$ :

$$
J(s, \Lambda)=\frac{m}{2 \pi} \sqrt{1+\frac{p^{2}}{m^{2}}}\left(\frac{2}{\pi} \Lambda+i p\right)
$$

At given order in the $1 / m$ expansion the renormalized loop function is introduced by the minimal number of subtractions at $p=-i z$. At leading chiral orders this leads to:

$$
\begin{aligned}
J_{R}(p, z) & =\frac{m}{2 \pi}(-z+i p) \\
& +\frac{1}{4 \pi m}\left(-\frac{3 z}{2} p^{2}-\frac{z^{3}}{2}+i p^{3}\right)+\mathcal{O}\left(Q^{4}\right) .
\end{aligned}
$$

Note that the leading term is renormalized via $J_{R}(-i z, z)=0$ whereas the subleading term requires a further renormalization condition.

\section{B.3 One-loop vertex}

The one-loop vertex function is introduced with 


$$
\begin{gathered}
\mathcal{V}_{\pi}^{(i)}\left(p_{2}, p_{1}\right)=\frac{i}{2} \int \frac{d^{4} l}{(2 \pi)^{4}}\left(S_{F}(P+l) \vec{\tau} \gamma_{5}\left(l+\frac{1}{2} \not l\right)\right)^{t} \tau_{2} \tau_{i} C \gamma_{5} \\
\cdot S_{\pi}\left(l+\frac{1}{2} Q\right) S_{F}(K-l) \vec{\tau} \gamma_{5}\left(l+\frac{1}{2} \not\right) \\
u^{t}\left(p_{2}\right) \mathcal{V}_{\pi}^{(i)}\left(p_{2}, p_{1}\right) u\left(p_{1}\right)=V_{\pi}(s) u^{t}\left(p_{2}\right) C \gamma_{5} \frac{1}{2} \tau_{2} \tau_{i} u\left(p_{1}\right)
\end{gathered}
$$

where $u\left(p_{1,2}\right)$ are the incoming nucleon spinors. The loop function is expressed in terms of the master functions

$$
V_{\pi}(s)=4 m^{2}\left(m_{\pi}^{2} I_{3, N}(s)-I_{2, N}(s)\right)+I_{1, \pi}-2 I_{1, N}+2 m_{\pi}^{2} I_{2}\left(m^{2}\right) .
$$

According to our renormalization procedure we first transform to the on-shell irreducible interaction kernel. This leads to the pion-exchange with pseudoscalar coupling which is known to be on-shell equivalent to the pseudo-vector coupling used in (B.27). The vertex evaluated with pseudo-scalar coupling agrees with the pseudo-vector result (B.28) up to the divergent terms $I_{1, \pi}-$ $2 I_{1, N}+2 m_{\pi}^{2} I_{2}\left(m^{2}\right)$. The latter terms are included in the local part of the on-shell irreducible kernel $\hat{K}=\hat{K}_{\pi}+\hat{K}_{\sigma}$ according to (44):

$$
\hat{K}_{\sigma}=\left(g(s)+\frac{g_{A}^{2} g(s)}{2 f_{\pi}^{2}}\left(I_{1, \pi}-2 I_{1, N}+2 m_{\pi}^{2} I_{2}\left(m^{2}\right)\right)\right) T_{1} \mathcal{P}_{1}+\cdots
$$

with the bare coupling function $g(s)$ of (14). Similarly the $\Delta I_{3, N}(s)$ and $\Delta I_{2, N}(s)$ terms in (B.28) may be absorbed into $\tilde{K}=\tilde{K}_{\pi}+\tilde{K}_{\sigma}$ according to $(46)$ :

$$
\begin{aligned}
\tilde{K}_{\sigma} & =\left(g(s)+\frac{g_{A}^{2} g(s)}{2 f_{\pi}^{2}}\left(I_{1, \pi}-2 I_{1, N}+2 m_{\pi}^{2} I_{2}\left(m^{2}\right)\right)\right. \\
& \left.+\frac{g_{A}^{2} g(s)}{2 f_{\pi}^{2}} 4 m^{2}\left(m_{\pi}^{2} \Delta I_{3, N}(s)-\Delta I_{2, N}(s)\right)\right) T_{1} \mathcal{P}_{1}+\cdots
\end{aligned}
$$

Note that in our regularization prescription the nucleonic tadpole $I_{1, N}$ is dropped whereas the pionic tadpole (see (B.21)) contributes at chiral order $Q$. The renormalized vertex function $V_{\pi, R}(s)$ follows upon a proper loop subtraction according to $(41,63)$ with

$$
\begin{aligned}
V_{\pi}(s) & =V_{\pi, R}(s)-\bar{c}(s) J(s)+I_{1, \pi}-2 I_{1, N}+2 m_{\pi}^{2} I_{2}\left(m^{2}\right) \\
V_{\pi, R}(s) & =\frac{m^{2} m_{\pi}}{8 \pi \sqrt{m^{2}+p^{2}}} \frac{m_{\pi}}{p} i \ln \left(1-i \frac{2 p}{m_{\pi}}\right), \quad \bar{c}(s)=\frac{1}{2} \frac{m^{2}}{m^{2}+p^{2}} .
\end{aligned}
$$

Note that the loop function $V_{\pi, R}(s)$ is most easily derived by first evaluating its imaginary part. The real part is then constructed with ease since 
$\sqrt{m^{2}+p^{2}} V_{\pi, R}\left(4 m^{2}+4 p^{2}\right)$ is an analytic function in the upper complex half $p$-plane.

\section{B.4 Two-loop bubble}

The two-loop bubble integral is

$$
J_{\pi}(s)=-\frac{i}{2} \operatorname{tr} \int \frac{d^{4} l}{(2 \pi)^{4}} \tau_{j} \tau_{2} \gamma_{5} C^{-1} S_{F}^{t}(P+l) \mathcal{V}_{\pi}^{(j)}(P+l, K-l) S_{F}(K-l)
$$

defined in terms of the full off-shell one-loop vertex function (B.27). Here we implicitly assume that the vertex (B.27) is evaluated with pseudo-scalar pion nucleon coupling according to $(44)$. We evaluate $J_{\pi}(s)$ by constructing its imaginary part and then recovering its real part by means of dispersion relations. The loop is written as a sum of three terms

$$
J_{\pi}(s)=\bar{J}_{\pi}(s)-\bar{c}(s) J^{2}(s)+J_{\pi}^{(\pi)}(s)
$$

where $J_{\pi}^{(\pi)}(s)$ is the unique contribution due to the pion production cut. The square of the one-loop bubble $J(s)$ in (B.33) takes care of the overlapping divergence structure of the two-loop function $J_{\pi}(s)$.

We first evaluate the loop-subtracted bubble function $\bar{J}_{\pi}(s)$ by imposing a dispersion relation on $s \bar{J}_{\pi}(s)$. Here we pull out the factor $1 / s^{\prime}$ in the dispersion relation in order to comply with our regularization scheme and (46). With $\bar{c}(s)$ as given in (B.31) the appropriate spectral density is readily derived

$$
\Im \bar{J}_{\pi}(s)=2 \rho_{J}(s) \Re V_{\pi, R}(s)
$$

in terms of the renormalized vertex function $V_{\pi, R}(s)$ of (B.31). The following representation can now be established

$$
\bar{J}_{\pi}(p)-\bar{J}_{\pi}(0)=i \frac{m p}{\pi} V_{\pi, R}^{(1)}(p)=-2 \frac{m^{2} m_{\pi}^{2}}{(4 \pi)^{2}} \ln \left(1-i \frac{2 p}{m_{\pi}}\right)
$$

where $\sqrt{s} V_{\pi, R}(s)=m V_{\pi, R}^{(1)}(p)$. Note that (B.35) shows that all $1 / m$ correction terms for $\bar{J}_{\pi}(p)-\bar{J}_{\pi}(0)$ cancel identically. The identity (B.35) follows from the Kramers-Kronig relations: 


$$
\begin{aligned}
i\left(V_{\pi, R}^{(1)}(p)-V_{\pi, R}^{(1)}(0)\right) & =\int_{-\infty}^{\infty} \frac{d \bar{p}}{\pi} \frac{p}{\bar{p}} \frac{\Re V_{\pi, R}^{(1)}(\bar{p})}{\bar{p}-p-i \epsilon} \\
& =\frac{\pi}{m p}\left(\bar{J}_{\pi}(p)-\bar{J}_{\pi}(0)\right)-i V_{\pi, R}^{(1)}(0), \\
\bar{J}_{\pi}(p)-\bar{J}_{\pi}(0) & =\int_{0}^{\infty} \frac{d \bar{p}^{2}}{\pi^{2}} \frac{p^{2}}{\bar{p}^{2}} \frac{m \bar{p}}{\bar{p}^{2}-p^{2}-i \epsilon} \Re V_{\pi, R}^{(1)}(\bar{p}) .
\end{aligned}
$$

This is an amazingly simple result. The two-loop bubble function is given in terms of the one-loop vertex function $V_{\pi, R}^{(1)}(p)$. Note that the loop function $\bar{J}_{\pi}(p)$ shows a log-divergence. In analogy to (B.23) the subtraction constant $\bar{J}_{\pi}(0)$ may be reconstructed by a cutoff regularized dispersion relation:

$$
\begin{aligned}
\bar{J}_{\pi}(0) & =m^{2} \int_{0}^{\Lambda^{2}} \frac{d \bar{p}^{2}}{\pi^{2}}\left(\frac{m}{\bar{p}}+\frac{\bar{p}}{m}\right) \Re V_{\pi, R}^{(1)}(\bar{p}) \\
& =\frac{m^{2} m_{\pi}^{2}}{(4 \pi)^{2}}\left(\ln \left(\frac{\Lambda^{2}}{m_{\pi}^{2}}\right)+\mathcal{O}\left(\frac{m_{\pi}}{\Lambda}, \frac{\Lambda^{2}}{m^{2}}\right)\right) .
\end{aligned}
$$

The renormalized loop follows now with $J_{\pi, R}(p, z)=\bar{J}_{\pi}(p)-\bar{J}_{\pi}(-i z)$ according to $(42)$.

We turn to the evaluation of the pion production term $J_{\pi}^{(\pi)}(s)$. This contribution is conceptually interesting since it involves a further small scale

$$
\mu_{\pi}^{2}=m m_{\pi}+\frac{m_{\pi}^{2}}{4}
$$

characterizing the the pion production process at $p>\mu_{\pi}$. The spectral density is systematically expanded in powers of $\left(p^{2}-\mu_{\pi}^{2}\right)^{n}$

$$
\rho_{J, \pi}^{(\pi)}(p)=m_{\pi}^{4} \Theta\left(p-\mu_{\pi}\right) \frac{\left(p^{2}-\mu_{\pi}^{2}\right)^{2}}{2(2 \pi)^{3} p^{4}}+\mathcal{O}\left(Q^{5}\right)
$$

where we count $p \sim \mu_{\pi} \sim Q$. We point out that our expansion of the pion production spectral function is in full analogy to the non relativistic expansion of the two nucleon unitarity cut where one expands in powers of the relative momentum $p$ also around the threshold value $p=0$. The real part is derived from the dispersion relation:

$$
J_{\pi, R}^{(\pi)}(p, z)=\frac{m_{\pi}^{4}}{(2 \pi)^{4}}\left(-\frac{\mu_{\pi}^{2}}{p^{2}}+\frac{\left(p^{2}-\mu_{\pi}^{2}\right)^{2}}{p^{4}} \ln \left|\frac{\mu_{\pi}^{2}}{\mu_{\pi}^{2}-p^{2}}\right|\right.
$$




$$
\begin{aligned}
& -\frac{\mu_{\pi}^{2}}{z^{2}}-\frac{\left(z^{2}+\mu_{\pi}^{2}\right)^{2}}{z^{4}} \ln \left|\frac{\mu_{\pi}^{2}}{\mu_{\pi}^{2}+z^{2}}\right| \\
& \left.+i \pi \Theta\left(p-\mu_{\pi}\right) \frac{\left(p^{2}-\mu_{\pi}^{2}\right)^{2}}{p^{4}}\right)+\mathcal{O}\left(Q^{5}\right)
\end{aligned}
$$

properly subtracted at $p^{2}=-z^{2}$. Note that the chiral order $J_{\pi, R}^{(\pi)}(p, z) \sim Q^{4}$ is deduced by applying simply Weinberg's counting rules. In other words due to the absence of an intermediate nucleon pair state there is no enhancement factor for the pion production spectral density.

\section{B.5 Pion production loops}

We consider the self energy correction to the one-loop bubble diagram

$$
\begin{gathered}
J_{\Sigma}(s)=-i \operatorname{tr} \int \frac{d^{4} l}{(2 \pi)^{4}} C^{-1} \gamma_{5} S_{F}\left(l+\frac{1}{2} w\right) \Sigma\left(l+\frac{1}{2} w\right) S_{F}\left(l+\frac{1}{2} w\right) \\
\gamma_{5} C S_{F}^{t}\left(-l+\frac{1}{2} w\right) \\
-i \operatorname{tr} \int \frac{d^{4} l}{(2 \pi)^{4}} C^{-1} \gamma_{5} S_{F}\left(l+\frac{1}{2} w\right) \gamma_{5} C S_{F}^{t}\left(-l+\frac{1}{2} w\right) \\
\Sigma^{t}\left(-l+\frac{1}{2} w\right) S_{F}^{t}\left(-l+\frac{1}{2} w\right)
\end{gathered}
$$

with $w^{2}=s$ and the self energy

$$
\Sigma(p)=\Sigma_{\pi N}-3 g_{A}^{2} \lambda^{2} i \int \frac{d^{4} l}{(2 \pi)^{4}} \gamma_{5} l / S_{F}(p+l) \gamma_{5} l / S_{\pi}(l)+c . t .
$$

receiving contributions from the pion nucleon sigma term $\Sigma_{\pi N} \simeq 45 \mathrm{MeV}$ and suitable counter terms. We also consider the two-loop function, $J_{V}(s)$, induced by the pion production vertex in (124):

$$
\begin{aligned}
J_{V}(s) & =-2 i \operatorname{tr} \int \frac{d^{4} l}{(2 \pi)^{4}} C^{-1} V\left(l+\frac{1}{2} w\right) S_{F}\left(l+\frac{1}{2} w\right) \gamma_{5} C S_{F}^{t}\left(-l+\frac{1}{2} w\right) \\
& -2 i \operatorname{tr} \int \frac{d^{4} l}{(2 \pi)^{4}} C^{-1} \gamma_{5} S_{F}\left(l+\frac{1}{2} w\right) \bar{V}\left(-l+\frac{1}{2} w\right) C S_{F}^{t}\left(-l+\frac{1}{2} w\right), \\
V(p) & =-i \int \frac{d^{4} l}{(2 \pi)^{4}} l S_{F}(p+l) \gamma_{5} l S_{\pi}(l) .
\end{aligned}
$$

with the vertex function $\bar{V}(p)=\gamma_{0} V^{\dagger}(p) \gamma_{0}$. Note that in $($ B.41,B.43) we suppress the isospin space but include isospin factors effectively. 
The two-loop functions $J_{\Sigma}(s)$ and $J_{V}(s)$ have two types of s-channel cuts. Cuts which do not separate the pion line simply give rise to a renormalization of the nucleon mass in the one-loop bubble $J(s)$ in case of $J_{\Sigma}(s)$ and a renormalization of the bare coupling in case of $J_{V}(s)$. They are effectively taken into account by using the physical nucleon mass in $J(s)$ and a renormalized bare coupling. The more interesting cuts with the pion line separated reflect the pion production process. We derive the leading contribution:

$$
\begin{aligned}
& \rho_{J, \Sigma}^{(\pi)}(p)=3 m_{\pi}^{4} \Theta\left(p-\mu_{\pi}\right) \frac{\left(p^{2}-\mu_{\pi}^{2}\right)^{2}}{2(2 \pi)^{3} p^{4}}+\mathcal{O}\left(Q^{5}\right), \\
& \rho_{J, V}^{(\pi)}(p)=m_{\pi}^{4} \Theta\left(p-\mu_{\pi}\right) \frac{\left(p^{2}-\mu_{\pi}^{2}\right)^{2}}{(2 \pi)^{3} p^{2} m}+\mathcal{O}\left(Q^{7}\right)
\end{aligned}
$$

and conclude $J_{\Sigma, R}^{(\pi)}(p, z)=3 J_{\pi, R}^{(\pi)}(p, z)+\mathcal{O}\left(Q^{5}\right)$ (see (B.40)). Again we confirm that Weinberg's counting rules can be applied for pion production cuts.

\section{B.6 Pion nucleon box}

Consider the pion nucleon box diagram

$$
\begin{array}{r}
B=-i \int \frac{d^{4} l}{(2 \pi)^{4}} S_{\pi}\left(l-\frac{1}{2} Q\right) \tau_{i} \gamma_{5}\left(l+\frac{1}{2} \phi\right) S_{F}(P-l) \tau_{j} \gamma_{5}\left(-l+\frac{1}{2} \not\right) \\
\otimes \tau_{i} \gamma_{5}\left(l+\frac{1}{2} \phi\right) S_{F}(K+l) \tau_{j} \gamma_{5}\left(-l+\frac{1}{2} \not\right) S_{\pi}\left(l+\frac{1}{2} Q\right)(\mathrm{B} .45)
\end{array}
$$

where the tensor product ' $\otimes$ ' is defined via the replacement rule

$$
\bar{\Gamma} \otimes \Gamma \rightarrow\left(\bar{u}\left(p_{2}^{\prime}\right) \bar{\Gamma} u\left(p_{2}\right)\right)\left(\bar{u}\left(p_{1}^{\prime}\right) \Gamma u\left(p_{1}\right)\right)
$$

with the incoming momenta $p_{1,2}$ and the outgoing momenta $p_{1,2}^{\prime}$ of the nucelons. On-shell projection simplifies the diagram considerably. The isospin one component, $B^{(1)}$, follows with:

$$
B^{(I=1)}=X^{\mu \nu}\left(\gamma_{\mu} \otimes \gamma_{\nu}\right)+\left(\gamma_{\mu} \otimes X^{\mu}+\bar{X}^{\mu} \otimes \gamma_{\mu}\right)+X
$$

where

$$
\begin{aligned}
X_{\mu \nu} & =16 m^{4} i \int \frac{d^{4} l}{(2 \pi)^{4}} l_{\mu} l_{\nu} S_{\pi}\left(l-\frac{1}{2} Q\right) S_{N}(P-l) S_{N}(K+l) S_{\pi}\left(l+\frac{1}{2} Q\right) \\
\bar{X}_{\mu} & =4 m^{2} i \int \frac{d^{4} l}{(2 \pi)^{4}} l_{\mu}(l+2 m) S_{\pi}\left(l-\frac{1}{2} Q\right) S_{N}(K+l) S_{\pi}\left(l+\frac{1}{2} Q\right)
\end{aligned}
$$




$$
\begin{gathered}
X_{\mu}=4 m^{2} i \int \frac{d^{4} l}{(2 \pi)^{4}} l_{\mu}(l-2 m) S_{\pi}\left(l-\frac{1}{2} Q\right) S_{N}(P-l) S_{\pi}\left(l+\frac{1}{2} Q\right) \\
X=-i \int \frac{d^{4} l}{(2 \pi)^{4}}(2 m-l) \otimes(2 m+l) S_{\pi}\left(l-\frac{1}{2} Q\right) S_{\pi}\left(l+\frac{1}{2} Q\right) .
\end{gathered}
$$

It is useful to introduce auxiliary loop functions $J_{i}(t)$ and $b_{i}(s, t)$ which exhaust the structure of $X_{\mu \nu}, X_{\mu}, \bar{X}_{\nu}$ and $X$ :

$$
\begin{aligned}
X_{\mu \nu} & =b_{1}(s, t) g^{\mu \nu}+\frac{b_{2}(s, t)}{m^{2}}(P+K)^{\mu}(P+K)^{\nu} \\
& +\frac{b_{3}(s, t)}{m^{2}}(P-K)^{\mu}(P-K)^{\nu}, \\
\bar{X}_{\mu} & =-\frac{1}{2} J_{1}^{(\pi)}(t) \gamma_{\mu}-\left(\frac{1}{2} J_{2}^{(\pi)}(t)+2 J_{4}^{(\pi)}(t)\right) \frac{K_{\mu}}{m}, \\
X_{\mu} & =-\frac{1}{2} J_{1}^{(\pi)}(t) \gamma_{\mu}-\left(\frac{1}{2} J_{2}^{(\pi)}(t)+2 J_{4}^{(\pi)}(t)\right) \frac{P_{\mu}}{m}, \\
X & =\frac{1}{3} J_{0}^{(\pi)}(t)\left(\gamma_{\mu} \otimes \gamma^{\mu}\right)-2 J_{3}^{(\pi)}(t)(1 \otimes 1) .
\end{aligned}
$$

Here we drop terms proportional to $Q_{\mu}$ or $Q_{\nu}$ since they would not contribute to the on-shell scattering matrix. Note that transforming to the on-shell irreducible one-pion exchange with pseudo-scalar coupling 'moves' all $J_{i}^{(\pi)}$ functions into the on-shell irreducible kernel. The invariant functions $b_{i}(s, t)$ follow easily after applying the substitution rule

$$
\begin{aligned}
l_{\mu} l_{\nu} & \rightarrow g_{\mu \nu}\left(l^{2}-\frac{(l \cdot Q)^{2}}{Q^{2}}-\frac{(l \cdot W)^{2}}{W^{2}}-\frac{(l \cdot(K-P))^{2}}{(K-P)^{2}}\right) \\
+ & \frac{W_{\mu} W_{\nu}}{W^{2}}\left(\frac{(l \cdot Q)^{2}}{Q^{2}}+2 \frac{(l \cdot W)^{2}}{W^{2}}+\frac{(l \cdot(K-P))^{2}}{(K-P)^{2}}-l^{2}\right) \\
+ & \frac{(K-P)_{\mu}(K-P)_{\nu}}{(K-P)^{2}}\left(\frac{(l \cdot Q)^{2}}{Q^{2}}+\frac{(l \cdot W)^{2}}{W^{2}}+2 \frac{(l \cdot(K-P))^{2}}{(K-P)^{2}}-l^{2}\right)
\end{aligned}
$$

in (B.49) with $W=P+K$ which is justified by covariance. The reduced box functions, $b_{i}(u, t)$, can now be expressed in terms of the master loop functions systematically:

$$
\begin{aligned}
b_{1}(s, t) & =16 m^{4}\left(\frac{\left(m_{\pi}^{2}-\frac{t}{2}\right)^{2}}{u}-m_{\pi}^{2}+\frac{t}{4}\right) I_{4, N}(s, t) \\
& +16 m^{4}\left(\frac{1}{2}-\frac{m_{\pi}^{2}-\frac{t}{2}}{u}\right) I_{3, N}(s)+16 m^{4} \frac{m_{\pi}^{2}-\frac{t}{2}}{u} I_{3, \pi}(t),
\end{aligned}
$$




$$
\begin{aligned}
b_{3}(s, t) & =16 \frac{m^{6}}{u}\left(m_{\pi}^{2}-\frac{t}{4}-2 \frac{\left(m_{\pi}^{2}-\frac{t}{2}\right)^{2}}{u}\right) I_{4, N}(s, t) \\
& -16 \frac{m^{6}}{u}\left(\frac{1}{2}-\frac{m_{\pi}^{2}}{u+t}-2 \frac{m_{\pi}^{2}-\frac{t}{2}}{u}\right) I_{3, N}(s) \\
& -32 \frac{m^{6}}{u} \frac{m_{\pi}^{2}-\frac{t}{2}}{u} I_{3, \pi}(t)-16 \frac{m^{6}}{u(u+t)}\left(I_{2, N}(s)-I_{2}\left(m^{2}\right)\right) \\
& -16 \frac{m^{6}}{u} \frac{m_{\pi}^{2}-\frac{t}{2}}{s+u} I_{3, \pi}(t)-16 \frac{m^{6}}{u(s+u)}\left(I_{2, \pi}(t)-I_{2}\left(m^{2}\right)\right)
\end{aligned}
$$

with $u=4 m^{2}-s-t$. The box function $b_{2}(s, t)$ can in fact be written in terms of $b_{1}(s, t)$ and $J_{4}^{(\pi)}(t)$ as follows:

$$
b_{2}(s, t)=-\frac{m^{2}}{s} b_{1}(s, t)+2 \frac{m^{2}}{s} J_{4}^{(\pi)}(t)
$$

It remains the evaluation of the reducible loops $J_{i}^{(\pi)}(t)$. Applying similar methods we derive:

$$
\begin{aligned}
J_{0}^{(\pi)}(t) & =\frac{1}{2} I_{1, \pi}-\left(m_{\pi}^{2}-\frac{t}{4}\right) I_{2, \pi}(t) \\
J_{1}^{(\pi)}(t) & =4 m^{2}\left(\frac{\left(m_{\pi}^{2}-\frac{1}{2} t\right)^{2}}{s+u}-m_{\pi}^{2}+\frac{t}{4}\right) I_{3, \pi}(t) \\
& +\frac{4 m^{2}}{s+u}\left(m_{\pi}^{2}-\frac{t}{2}\right)\left(I_{2, \pi}(t)-I_{2}\left(m^{2}\right)\right)+2 m^{2} I_{2}\left(m^{2}\right), \\
J_{2}^{(\pi)}(t) & =\frac{16 m^{4}}{s+u}\left(m_{\pi}^{2}-\frac{t}{4}-3 \frac{\left.\left(m_{\pi}^{2}-\frac{1}{2} t\right)^{2}\right)}{s+u}\right) I_{3, \pi}(t) \\
& -3 \frac{16 m^{4}}{(s+u)^{2}}\left(m_{\pi}^{2}-\frac{t}{2}\right) I_{2, \pi}(t) \\
& +\frac{16 m^{4}}{s+u}\left(3 \frac{m_{\pi}^{2}-\frac{1}{2} t}{s+u}-\frac{1}{2}+\frac{m_{\pi}^{2}}{2 m^{2}}\right) I_{2}\left(m^{2}\right)+\frac{8 m^{2}}{s+u}\left(I_{1, \pi}-I_{1, N}\right), \\
J_{3}^{(\pi)}(t) & =-2 m^{2} I_{2, \pi}(t) \\
J_{4}^{(\pi)}(t) & =8 \frac{m^{4}}{s+u}\left(m_{\pi}^{2}-\frac{1}{2} t\right) I_{3, \pi}(t)+8 \frac{m^{4}}{s+u}\left(I_{2, \pi}(t)-I_{2}\left(m^{2}\right)\right)
\end{aligned}
$$

with $s+u=4 m^{2}-t$. Finally it is convenient to decompose the on-shell projected pion nucleon box into our Dirac tensors $T_{i}$, with simple exchange properties 


$$
B\left(p_{2}^{\prime}, p_{2} ; p_{1}^{\prime}, p_{1}\right)=\mathcal{P}_{0} \sum_{i=1}^{5} B_{i}^{(0)}(u, t) T_{i}+\mathcal{P}_{1} \sum_{i=1}^{5} B_{i}^{(1)}(u, t) T_{i}
$$

The exchange box diagram is now deduced by properly exchanging $u$ and $t$ in the reduced functions $B_{i}(u, t)$ (see $(56)$ ). Note that the isospin zero amplitudes are $B_{i}^{(0)}(u, t)=9 B_{i}^{(1)}(u, t)$. Here we provide the necessary on-shell tensor identities (see [24]):

$$
\begin{aligned}
(\not K-m) \otimes(m-\not P) & =\left(\frac{1}{4} t-\frac{3}{4} u\right) T_{3}+\frac{1}{4}(u+t) T_{4}+\frac{1}{4}(u+t) T_{5} \\
\not K \otimes m+m \otimes \not P & =\left(m^{2}-\frac{1}{4}(u-t)\right) T_{1}+\left(m^{2}-\frac{1}{4}(u+t)\right) T_{2} \\
& -\left(\frac{1}{2} m^{2}+\frac{1}{4}(u-t)\right) T_{3}-\left(\frac{1}{2} m^{2}-\frac{1}{4}(u+t)\right) T_{4} \\
& +\frac{1}{2} m^{2} T_{5} \\
\not K \otimes \not P & =\left(\frac{1}{2} m^{2}-\frac{1}{4}(u-t)\right) T_{1}+\left(\frac{1}{2} m^{2}-\frac{1}{4}(u+t)\right) T_{2} \\
& -\left(\frac{3}{4} m^{2}-\frac{1}{2} u\right) T_{3}-\frac{1}{4} m^{2} T_{4} \\
& +\left(\frac{3}{4} m^{2}-\frac{1}{4}(u+t)\right) T_{5}, \\
(\not K+m) \otimes(m+\not P) & =\left(2 m^{2}-\frac{1}{2}(u-t)\right) T_{1}+\left(2 m^{2}-\frac{1}{2}(u+t)\right) T_{2} \\
& -\left(m^{2}-\frac{1}{4}(u+t)\right)\left(T_{3}+T_{4}-T_{5}\right) .
\end{aligned}
$$

We collect the leading terms of chiral order one:

$$
\begin{aligned}
B_{1}^{(1)}(u, t) & =-2 m^{2}\left(m_{\pi}^{2}-\frac{t}{2}\right) I_{3, \pi}(t)-\frac{t}{4 m^{2}} b_{1}(s, t) \\
& +2 m^{2} I_{2}\left(m^{2}\right)-\frac{11}{12} I_{1, \pi}+\cdots, \\
B_{2}^{(1)}(u, t) & =-2 m^{2}\left(m_{\pi}^{2}-\frac{t}{2}\right) I_{3, \pi}(t)+2 m^{2} I_{2}\left(m^{2}\right)-\frac{11}{12} I_{1, \pi}+\cdots, \\
B_{3}^{(1)}(u, t) & =-\frac{3}{4} b_{1}(s, t)+\frac{t-3 u}{4 m^{2}} b_{3}(s, t)+\cdots, \\
B_{4}^{(1)}(u, t) & =\frac{1}{4} b_{1}(s, t)+\frac{u+t}{4 m^{2}} b_{3}(s, t)+\cdots, \\
B_{5}^{(1)}(u, t) & =\frac{1}{4} b_{1}(s, t)+\frac{u+t}{4 m^{2}} b_{3}(s, t)+\cdots
\end{aligned}
$$

where the dots represent further terms with chiral power $\sim Q^{2}$ not relevant in this work.

It is instructive to consider also the pion-nucleon box diagram defined with a pseudo-scalar pion-nucleon coupling vertex (PS). In our calculation the pseudo-scalar box follows formally from the pseudo-vector box diagram defined in (B.45) by dropping the $X_{\mu}, \bar{X}_{\mu}$ and $X$ terms in (B.47). We observe that the difference of pseudo-vector and pseudo-scalar box diagram carries leading chiral order $Q^{0}$ : 


$$
\begin{aligned}
B_{1}^{(1)}(u, t)-B_{1}^{(1, P S)}(u, t) & =-4 m^{2}\left(m_{\pi}^{2}-\frac{t}{2}\right) I_{3, \pi}(t)-2 m^{2} I_{2, \pi}(t) \\
& +4 m^{2} I_{2}\left(m^{2}\right)-\frac{11}{12} I_{1, \pi}+\mathcal{O}\left(Q^{2}\right) \\
B_{2}^{(1)}(u, t)-B_{2}^{(1, P S)}(u, t) & =-4 m^{2}\left(m_{\pi}^{2}-\frac{t}{2}\right) I_{3, \pi}(t)-2 m^{2} I_{2, \pi}(t) \\
& +4 m^{2} I_{2}\left(m^{2}\right)-\frac{11}{12} I_{1, \pi}+\mathcal{O}\left(Q^{2}\right)
\end{aligned}
$$

This implies that the on-shell irreducible kernel $\hat{K}=\sum_{i=1}^{5} \hat{g}_{i}(u, t) T_{i} \mathcal{P}_{1}$ of (44) receives a non-local contribution. For example:

$$
\begin{aligned}
\hat{g}_{1}(u, t) & =\frac{1}{2} g_{A}^{4} \lambda^{4}\left(B_{1}^{(1)}(u, t)-B_{1}^{(1, P S)}(u, t)\right) \\
& +\frac{1}{2} g_{A}^{4} \lambda^{4}\left(B_{1}^{(1)}(t, u)-B_{1}^{(1, P S)}(t, u)\right) .
\end{aligned}
$$

The non-local terms of (B.58) are then included in $\hat{K}_{\pi}$ where $\hat{K}=\hat{K}_{\pi}+$ $\hat{K}_{\sigma}$. The pionic tadpole in (B.57) is included in $\hat{K}_{\sigma}$ and absorbed into the coupling function $g(s)$. Since both $K_{\pi}$ and $\hat{K}_{\pi}$ are treated in perturbation theory our procedure (44) defines a systematic procedure to absorb divergent terms, which are not associated with the s-channel unitartity cut, into the local bare coupling function $\hat{K}_{\sigma}$. At subleading order it leads to the inclusion of the pionic tadpole $I_{1, \pi}$ into $g(s)$.

We close our essay on the pion-nucleon box diagram with the relevant combination for s-wave scattering in the spin singlet channel:

$$
\begin{aligned}
T_{\left[{ }^{1} S_{0}\right]}(u, t) & =a_{1}^{(1)}(u, t)-\frac{u-t}{s} a_{4}^{(1)}(u, t)-\frac{u+t}{s} a_{5}^{(1)}(u, t) \\
& =2 g_{A}^{4} \lambda^{4} m_{\pi}^{4} m^{2} I_{4, N}(s, t)-4 g_{A}^{4} \lambda^{4} m_{\pi}^{2} m^{2} I_{3, N}(s) \\
& +2 g_{A}^{4} \lambda^{4} m^{2} I_{2, N}(s)+m^{2} \mathcal{O}\left(\frac{1}{m^{2}}\right)+(u \leftrightarrow t)
\end{aligned}
$$

where we now dropped the pionic tadpole contribution.

\section{B.7 Two-loop vertex}

The two-loop vertex function is

$$
\begin{gathered}
\mathcal{V}_{\pi \pi}^{(i)}\left(p_{2}, p_{1}\right)=i \int \frac{d^{4} l}{(2 \pi)^{4}}\left(S_{F}(P+l) \vec{\tau} \gamma_{5}\left(l+\frac{1}{2} \phi\right)\right)^{t} \mathcal{V}_{\pi}^{(i)}(P+l, K-l) \\
\cdot S_{\pi}\left(l+\frac{1}{2} Q\right) S_{F}(K-l) \vec{\tau} \gamma_{5}\left(l+\frac{1}{2} \not\right) \\
u^{t}\left(p_{2}\right) \mathcal{V}_{\pi \pi}^{(i)}\left(p_{2}, p_{1}\right) u\left(p_{1}\right)=V_{\pi \pi}(s) u^{t}\left(p_{2}\right)\left(C \gamma_{5} \frac{1}{2} \tau_{2} \tau_{i}\right) u\left(p_{1}\right)
\end{gathered}
$$


We evaluate $V_{\pi \pi}(s)$ by imposing a dispersion relation on the renormalized vertex $s V_{\pi \pi, R}(s)$ with

$$
\begin{aligned}
V_{\pi \pi}(s) & =V_{\pi \pi, R}(s)-\bar{c}(s) J(s) V_{\pi, R}(s)-\bar{c}(s)\left(\bar{J}_{\pi}(s)+J_{\pi}^{(\pi)}(s)\right) \\
& +\bar{c}^{2}(s) J^{2}(s)+V_{\pi \pi, R}^{(\pi)}(s)
\end{aligned}
$$

Our renormalization via loop subtraction keeps track of the overlapping divergency structure of the vertex function $V_{\pi \pi}(s)$. The unique contribution from the one-pion production cut is denoted by $V_{\pi \pi, R}^{(\pi)}(s)$. With $\bar{c}(s)$ given in (B.31) the renormalized loop functions $V_{\pi \pi, R}(s)$ and $V_{\pi \pi, R}^{(\pi)}(s)$ are finite. Note that the pion production contribution $V_{\pi \pi, R}^{(\pi)}(s)$ is renormalized by the proper subtraction of the pion production loop $J_{\pi}^{(\pi)}(s)$. From (B.40) we expect $V_{\pi \pi, R}^{(\pi)}(s)$ to carry chiral order $Q^{4}$. The spectral function of $V_{\pi \pi, R}(s)$ follows in terms of the renormalized one-loop vertex $V_{\pi, R}(s)$ and an effective pion-nucleon box $B_{\pi \pi}(s)$ :

$$
\begin{aligned}
\Im V_{\pi \pi, R}(s) & =\left(\Im V_{\pi, R}(s)\right)\left(\Re V_{\pi, R}(s)\right)+(\Im J(s))\left(\Re B_{\pi \pi}(s)\right), \\
B_{\pi \pi}(s) & =\left.2 m^{2} m_{\pi}^{4} \int_{-1}^{1} \frac{d x}{2} I_{4, N}(s, t)\right|_{t=-2 p^{2}(1-x)}+\mathcal{O}\left(Q^{2}\right)
\end{aligned}
$$

with $s=4\left(m^{2}+p^{2}\right)$. Note that the function $B_{\pi \pi}(s)$ coincides with the s-wave projected pion-nucleon box diagram $R_{\pi \pi}(p)$ (see (80)) as should be expected. The renormalized loop is decomposed into its chiral moments:

$$
V_{\pi \pi, R}(s)=\sum_{n=2}^{\infty} V_{\pi \pi, R}^{(n)}(p)
$$

where $V_{\pi \pi, R}^{(n)}(p)$ carries chiral power $n$. The real part of the two-loop function $V_{\pi \pi, R}^{(2)}(p)$ can be easily derived without performing further integrations. Recall that $V_{\pi \pi, R}^{(2)}(p)$ satisfies a dispersion relation in $p$ :

$$
V_{\pi \pi, R}^{(2)}(p)=\int_{4 m^{2}}^{\infty} \frac{d s^{\prime}}{\pi} \frac{\Im V_{\pi \pi, R}^{(2)}\left(p^{\prime}\right)}{s^{\prime}-s-i \epsilon}=\int_{-\infty}^{\infty} \frac{d p^{\prime}}{\pi} \frac{\Im V_{\pi \pi, R}^{(2)}\left(p^{\prime}\right)}{p^{\prime}-p-i \epsilon}
$$

Therefore, if considered as a function of $p$ with $s=4\left(m^{2}+p^{2}\right)$, the chiral moment $V_{\pi \pi, R}^{(2)}(p)$ is analytic in the upper complex half plane. We note that the full vertex function $V_{\pi \pi}(s)$ on the other hand is not analytic in the upper complex half plane if considered as a function of $\mathrm{p}$. It shows branch points at $p= \pm i m$ which are systematically eliminated performing the $1 / m$ expansion. 
Start with the first term in (B.62). The result can immediately be deduced from the simple identity:

$$
\Im \ln ^{2}\left(1-2 i \frac{p}{m_{\pi}}\right)=-\arctan \left(2 \frac{p}{m_{\pi}}\right) \ln \left(1+4 \frac{p^{2}}{m_{\pi}^{2}}\right) .
$$

The second term follows with the somewhat more involved identity:

$$
\begin{aligned}
\Re R_{\pi \pi}(p)=\frac{m m_{\pi}}{4 \pi} \frac{m_{\pi}^{3}}{8 p^{3}} \Im\left(\operatorname{Li}_{2}\left(\frac{1}{2}+\frac{i p}{m_{\pi}}\right)-2 \operatorname{Li}_{2}\left(\frac{1}{2} \frac{m_{\pi}}{m_{\pi}-i p}\right)+\frac{\pi^{2}}{12}\right. \\
\left.-\ln ^{2}\left(\frac{m_{\pi}-2 i p}{2 m_{\pi}-2 i p}\right)+\frac{1}{2} \ln ^{2}\left(\frac{1}{2}-\frac{i p}{m_{\pi}}\right)\right) .
\end{aligned}
$$

For both terms we succeed in writing the spectral function as the imaginary part of an analytic function with branch cuts only on the lower complex half plane. Thus it is straightforward to reconstruct the full analytic function. We collect all terms and display our result ${ }^{9}$ :

$$
\begin{aligned}
V_{\pi \pi, R}^{(2)}(p)=\frac{m^{2} m_{\pi}^{2}}{(4 \pi)^{2}} & \frac{m_{\pi}^{2}}{4 p^{2}}\left(\operatorname{Li}_{2}\left(\frac{1}{2}+\frac{i p}{m_{\pi}}\right)-2 \operatorname{Li}_{2}\left(\frac{1}{2} \frac{m_{\pi}}{m_{\pi}-i p}\right)+\operatorname{Li}_{2}\left(\frac{1}{2}\right)\right. \\
- & \left.\ln ^{2}\left(\frac{m_{\pi}-2 i p}{2 m_{\pi}-2 i p}\right)-\ln \left(\frac{1}{2}-\frac{i p}{m_{\pi}}\right) \ln 2\right) .
\end{aligned}
$$

\section{B.8 Three-loop bubble}

We turn to the three-loop bubble

$$
J_{\pi \pi}(s)=-\frac{i}{2} \operatorname{tr} \int \frac{d^{4} l}{(2 \pi)^{4}} \tau_{j} \tau_{2} \gamma_{5} C^{-1} S_{F}^{t}(P+l) \mathcal{V}_{\pi \pi}^{(j)}(P+l, K-l) S_{F}(K-l)
$$

which is defined in terms of the full off-shell two-loop vertex of (B.60). We introduce the loop-subtracted bubble function, $\bar{J}_{\pi \pi}(s)$, with

$$
J_{\pi \pi}(s)=\bar{J}_{\pi \pi}(s)-2 \bar{c}(s) J(s) \bar{J}_{\pi}(s)+\bar{c}^{2}(s) J^{3}(s)+\cdots
$$

where we dropped terms of suppressed chiral orders induced by one and twopion production. The loop $\bar{J}_{\pi \pi}(s)$ is decomposed into its chiral moments:

9 Note the identity: $\operatorname{Li}_{2}\left(\frac{1}{2}\right)=\frac{\pi^{2}}{12}-\frac{1}{2} \ln ^{2} 2$ 


$$
\bar{J}_{\pi \pi}(s)=\sum_{n=3}^{\infty} \bar{J}_{\pi \pi}^{(n)}(p)
$$

where $\bar{J}_{\pi \pi}^{(n)}(p)$ carries chiral power $n$. The spectral function is expressed in terms of the renormalized one and two-loop vertex functions $V_{\pi, R}(s)$ and $V_{\pi \pi, R}(s)$

$$
\Im \bar{J}_{\pi \pi}(s)=2(\Im J(s))\left(\Re V_{\pi \pi, R}(s)\right)+(\Im J(s))\left(V_{\pi, R}(s) V_{\pi, R}^{*}(s)\right) .
$$

For the leading moment $\bar{J}_{\pi \pi}^{(3)}(p)$ we find in analogy to (B.35) the relation:

$$
\left.\bar{J}_{\pi \pi}^{(3)}(p)-\bar{J}_{\pi \pi}^{(3)}(0)=i \frac{m p}{2 \pi}\left(2 V_{\pi \pi, R}^{(2)}(p)+\left|V_{\pi, R}^{(1)}\right|_{c}^{2}(p)\right)\right)
$$

where we introduced the proper analytic continuation $\left|V_{\pi, R}^{(1)}\right|_{c}^{2}(p)$ of $\left|V_{\pi, R}^{(1)}(p)\right|^{2}$ with:

$$
\begin{aligned}
V_{\pi, R}^{(1)}(p) V_{\pi, R}^{(1), *}(p) & =\Re\left|V_{\pi, R}^{(1)}\right|_{c}^{2}(p) \\
& =\frac{m^{2} m_{\pi}^{2}}{(4 \pi)^{2}} \frac{m_{\pi}^{2}}{2 p^{2}} \Re\left(\operatorname{Li}_{2}\left(\frac{1}{2}\right)-\operatorname{Li}_{2}\left(\frac{1}{2}+\frac{i p}{m_{\pi}}\right)\right. \\
& \left.+\ln (2) \ln \left(1-2 \frac{i p}{m_{\pi}}\right)+\frac{i p}{m_{\pi}} \ln (16)\right) .
\end{aligned}
$$

We collect all terms and present the leading chiral order contribution

$$
\begin{aligned}
\bar{J}_{\pi \pi}^{(3)}(p)-\bar{J}_{\pi \pi}^{(3)}(0) & =-i \frac{m^{3} m_{\pi}^{3}}{(4 \pi)^{3}} \frac{m_{\pi}}{p}\left(2 \operatorname{Li}_{2}\left(\frac{1}{2} \frac{m_{\pi}}{m_{\pi}-i p}\right)\right. \\
& \left.-\frac{i p}{m_{\pi}} \ln (16)+\ln ^{2}\left(\frac{m_{\pi}-2 i p}{2 m_{\pi}-2 i p}\right)-\frac{\pi^{2}}{6}\right) .
\end{aligned}
$$

Note that the loop function $\bar{J}_{\pi \pi}^{(3)}(p)$ is convergent with

$$
\bar{J}_{\pi \pi}^{(3)}(0)=\int_{0}^{\infty} \frac{d \bar{p}^{2}}{\pi} \frac{\Im \bar{J}_{\pi \pi}^{(3)}(\bar{p})}{\bar{p}^{2}}=\ln (16) \frac{m^{3} m_{\pi}^{3}}{(4 \pi)^{3}}
$$

The integral (B.75) follows from (B.74) and the observation $\bar{J}_{\pi \pi}^{(3)}(\infty)=0$. The leading order renormalized three-loop function is $J_{\pi \pi, R}(p, z)=\bar{J}_{\pi \pi}^{(3)}(p)-$ $\bar{J}_{\pi \pi}^{(3)}(-i z)$. 


\section{NNLO terms in CE scheme}

In this appendix we systematically collect all terms of chiral order one in $\mathrm{CE}$ scheme. We emphasize that again explicit calculation shows that no $c$ dependence survives at this order. The auxiliary coupling function $g_{H}^{-1}$ receives the contributions

$$
\begin{aligned}
g_{H}^{-1}\left(s, m_{\pi} ; c\right) & =g_{\sigma, S}^{-1}\left(s, m_{\pi} ; c\right)-J\left(-i z_{0}\right)-g_{A}^{2} \lambda^{2} J_{\pi, S}\left(-i z_{0}\right) \\
& -g_{A}^{4} \lambda^{4} J_{\pi \pi, S}\left(-i z_{0}\right)-\left(c-\frac{1}{2}\right)^{3} g_{A}^{6} \lambda^{6} J^{4}\left(-i z_{0}\right)+\cdots
\end{aligned}
$$

where we included the anomalous contribution from $J_{\pi \pi \pi, S}$ according to (73). It is convenient to resolve the structure of the subtraction constants $J_{\pi, S}\left(-i z_{0}\right)$ and $J_{\pi \pi, S}\left(-i z_{0}\right)$ with:

$$
\begin{aligned}
g_{A}^{4} \lambda^{4} J_{\pi, S}\left(-i z_{0}\right) & =\left(c-\frac{1}{2}\right) \bar{\kappa}_{J}^{2}\left(z_{0}\right)+g_{A}^{2} \lambda^{2}\left(\bar{\kappa}_{J, \pi}\left(z_{0}\right)+\kappa_{J, \pi}^{(\pi)}\left(z_{0}\right)\right) \\
g_{A}^{6} \lambda^{6} J_{\pi \pi, S}\left(-i z_{0}\right) & =\left(c-\frac{1}{2}\right) \bar{\kappa}_{J}\left(z_{0}\right)\left(\left(c-\frac{1}{2}\right) \bar{\kappa}_{J}^{2}\left(z_{0}\right)+2 g_{A}^{2} \lambda^{2} \bar{\kappa}_{J, \pi}\left(z_{0}\right)\right) \\
& +g_{A}^{2} \lambda^{2} \bar{\kappa}_{J, \pi \pi}\left(z_{0}\right)
\end{aligned}
$$

where we introduce $\bar{\kappa}_{J}\left(z_{0}\right)=g_{A}^{2} \lambda^{2} J\left(-i z_{0}\right), \bar{\kappa}_{J, \pi}\left(z_{0}\right)=g_{A}^{2} \lambda^{2} \bar{J}_{\pi}\left(-i z_{0}\right)$ and $\bar{\kappa}_{J, \pi \pi}\left(z_{0}\right)=g_{A}^{4} \lambda^{4} \bar{J}_{\pi \pi}\left(-i z_{0}\right)$. For later convenience it is appropriate to generalize here our notation and introduce further dimensionless coefficients $\bar{\kappa}_{J, \pi^{k}}^{(l)}(z)$ and $\bar{\kappa}_{V, \pi^{k}}^{(l)}(z)$ :

$$
\begin{aligned}
& \bar{\kappa}_{J, \pi^{k}}^{(l)}(z)=g_{A}^{2 k} \lambda^{2 k}\left(\frac{2 \pi}{i m}\right)^{l} \frac{1}{l !} \partial_{p}^{l} J_{\pi^{k}, R}(-i z, z), \\
& \bar{\kappa}_{V, \pi^{k}}^{(l)}(z)=g_{A}^{2 k} \lambda^{2 k}\left(\frac{2 \pi}{i m}\right)^{l} \frac{1}{l !} \partial_{p}^{l} V_{\pi^{k}, R}(-i z) .
\end{aligned}
$$

The auxiliary coupling $g_{H}$ does show an explicit $c$-dependence at chiral or$\operatorname{der} Q^{3}$. However such terms are canceled by a contribution to the scattering amplitude with an explicit $c$-dependence

$$
T_{\left[{ }^{1} S_{0}\right]}(u, t)=4 \bar{\kappa}_{J}\left(c-\frac{1}{2}\right) \frac{g_{H}^{-1}(s)}{J_{R}^{2}(p, z)}+\cdots .
$$

The cancellation follows from the intermediate result

$$
g_{A}^{2} \lambda^{2} g_{H}^{-1}(s)-2 \bar{\kappa}_{J}\left(z_{0}\right)\left(c-\frac{1}{2}\right) g_{H}^{-1}(s)=g_{A}^{2} \lambda^{2} g_{R}^{-1}(p)+\mathcal{O}\left(Q^{4}\right)
$$


which considers the relevant combination. Again our result (C.5) relies on the leading order expression (92). We decompose the amplitude in its chiral moments $T_{\left[{ }^{1} S_{0}\right]}(p)=\sum_{n=-1}^{\infty} T_{\left[{ }^{1} S_{0}\right]}^{(n)}(p)$ and display our complete collection of terms with chiral power one:

$$
\begin{aligned}
T_{\left[{ }^{1} S_{0}\right]}^{(1)}(u, t) & =2 \frac{\bar{\kappa}_{J, \pi \pi}\left(z_{0}\right)}{J_{R}^{2}(p, z)}+2 \bar{\zeta}_{3} \frac{m}{2 \pi} \frac{m_{\pi}^{3}}{J_{R}^{2}(p, z)}-2 \bar{\zeta}_{2} m_{\pi}^{2} \frac{m}{2 \pi} \frac{\bar{\kappa}_{J, \pi}^{(1)}\left(z_{0}\right)}{J_{R}^{2}(p, z)} \\
& -2 \frac{g_{R}^{-2}(p)}{J_{R}^{3}(p, z)}-2\left(\bar{\zeta}_{2} m_{\pi}^{2}\right)^{2} \frac{m^{2}}{4 \pi^{2}} \frac{1}{J_{R}^{3}(p, z)} \\
& +4 \bar{\zeta}_{2} m_{\pi}^{2} \frac{m}{2 \pi} \frac{g_{R}^{-1}(p)}{J_{R}^{3}(p, z)}-2 g_{A}^{4} \lambda^{4}\left(2 \frac{V_{\pi \pi, R}(p)}{J_{R}\left(p, z_{0}\right)}-\frac{J_{\pi \pi, R}\left(p, z_{0}\right)}{J_{R}^{2}\left(p, z_{0}\right)}\right) \\
& -4 g_{A}^{2} \lambda^{2} \frac{g_{R}^{-1}(p)}{J_{R}(p, z)}\left(\frac{V_{\pi, R}(p)}{J_{R}\left(p, z_{0}\right)}-\frac{J_{\pi, R}\left(p, z_{0}\right)}{J_{R}^{2}\left(p, z_{0}\right)}\right) \\
& -2 \frac{g_{A}^{4} \lambda^{4}}{J_{R}^{3}\left(p, z_{0}\right)}\left(J_{R}\left(p, z_{0}\right) V_{\pi, R}(p)-J_{\pi, R}\left(p, z_{0}\right)\right)^{2} \\
& +g_{A}^{4} \lambda^{4}\left(B_{\pi \pi}(u, t)+B_{\pi \pi}(t, u)\right) .
\end{aligned}
$$

Our result (C.6) involves the previously given renormalized one-loop vertex $V_{\pi, R}(p) \sim Q$ and two loop bubble $J_{\pi, R}\left(p, z_{0}\right) \sim Q^{2}$. It further requires the renormalized two-loop vertex $V_{\pi \pi, R}(p) \sim Q^{2}$ and the three-loop bubble $J_{\pi \pi, R}\left(p, z_{0}\right) \sim Q^{3}$. Again all higher order pole terms must cancel. This requirement confirms $\bar{\zeta}_{2}=\zeta_{2}^{(0)}$ and determines the hitherto unknown coefficients $\bar{\zeta}_{3}$

$$
0=\frac{\bar{\kappa}_{J, \pi \pi}\left(z_{0}\right)}{m_{\pi}^{3}}+\bar{\zeta}_{2} \frac{\bar{\kappa}_{J, \pi}^{(1)}\left(z_{0}\right)}{m_{\pi}} \frac{m}{2 \pi}+\bar{\zeta}_{3} \frac{m}{2 \pi}
$$

in terms of the known parameters $\bar{\zeta}_{2}$ and $\bar{\kappa}_{J, \pi}^{(1)}\left(z_{0}\right), \bar{\kappa}_{J, \pi \pi}\left(z_{0}\right)^{10}$.

We make the analytic structure of our result (C.6) more explicit by expressing our result in terms of residual functions $R_{J, \pi^{k}}^{(n)}(p, z)$ and $R_{V, \pi^{k}}^{(n)}(p, z)$ which are defined

$$
\begin{aligned}
g_{A}^{2 k} \lambda^{2 k} J_{\pi^{k}, R}(p, z) & =g_{A}^{2 k} \lambda^{2 k} J_{R}^{n}(p, z) R_{J, \pi^{k}}^{(n)}(p, z)+\sum_{l=0}^{n-1} \bar{\kappa}_{J, \pi^{k}}^{(l)}(z) J_{R}^{l}(p, z) \\
g_{A}^{2 k} \lambda^{2 k} V_{\pi^{k}, R}(p) & =g_{A}^{2 k} \lambda^{2 k} J_{R}^{n}(p, z) R_{V, \pi^{k}}^{(n)}(p, z)
\end{aligned}
$$

$\overline{10}$ Note that here we suppress a finite renormalization of $\zeta_{0}^{(2)} \rightarrow \zeta_{0}^{(2)}\left(1-2 z_{0} \zeta_{2}^{(0)}\right)$. This renormalization leads effectively to $g_{R}^{-1} \sim \zeta_{2}^{(0)}\left(p^{2}+z^{2}\right)$. 


$$
+\sum_{l=0}^{n-1} \bar{\kappa}_{V, \pi^{k}}^{(l)}(z) J_{R}^{l}(p, z)
$$

in terms of the coefficients $\bar{\kappa}_{J, \pi^{k}}^{(l)}(z)$ and $\bar{\kappa}_{V, \pi^{k}}^{(l)}(z)$ of (C.3). This leads to the central result of this appendix

$$
\begin{aligned}
T_{\left[{ }^{1} S_{0}\right]}^{(1)}(p) & =\frac{4 \pi}{m} \frac{i w^{(2)}}{p+i z}+\frac{4 \pi}{m} \zeta_{2}^{(0)}\left(2 \bar{\kappa}_{V, \pi}^{(0)}\left(z_{0}\right)-2 \bar{\kappa}_{J, \pi}^{(1)}\left(z_{0}\right)-3 \zeta_{2}^{(0)} z_{0}\right) \\
& -\frac{4 \pi}{m}\left(\zeta_{2}^{(0)}\right)^{2} i p+2 g_{A}^{4} \lambda^{4} R_{\pi \pi}(p) \\
& +4 g_{A}^{2} \lambda^{2} \zeta_{0}^{(2)} m_{\pi}^{2} \frac{m}{2 \pi}\left(R_{J, \pi}^{(3)}\left(p, z_{0}\right)-R_{V, \pi}^{(2)}\left(p, z_{0}\right)\right) \\
& -4 g_{A}^{2} \lambda^{2}\left(R_{J, \pi}^{(2)}\left(p, z_{0}\right)-R_{V, \pi}^{(1)}\left(p, z_{0}\right)\right) \zeta_{2}^{(0)}\left(z_{0}+i p\right) \\
& +4 g_{A}^{2} \lambda^{2}\left(\bar{\kappa}_{V, \pi}^{(0)}\left(z_{0}\right)-\bar{\kappa}_{J, \pi}^{(1)}\left(z_{0}\right)\right)\left(R_{J, \pi}^{(2)}\left(p, z_{0}\right)-R_{V, \pi}^{(1)}\left(p, z_{0}\right)\right) \\
& +2 g_{A}^{4} \lambda^{4}\left(R_{J, \pi \pi}^{(2)}\left(p, z_{0}\right)-2 R_{V, \pi \pi}^{(1)}\left(p, z_{0}\right)\right) \\
& +2 g_{A}^{4} \lambda^{4}\left(z_{0}-i p\right) \frac{m}{2 \pi}\left(R_{V, \pi}^{(1)}\left(p, z_{0}\right)-R_{J, \pi}^{(2)}\left(p, z_{0}\right)\right)^{2}
\end{aligned}
$$

where we decomposed the residuum $w=\sum_{n=0}^{\infty} w^{(n)}$ into its chiral moments. In (C.9) we encounter the reduced functions $R_{V, \pi \pi}^{(1)}, R_{J, \pi \pi}^{(2)}$ and $R_{V, \pi}^{(2)}, R_{J, \pi}^{(3)}$ defined according to (100). Note that all terms in (C.9) are regular at $p=-i z$ and $p=-i z_{0}$ except the first one. The contribution from the pion nucleon box diagram sits in $R_{\pi \pi}(p)$ (see (80)). With (C.9) we arrive at the desired representation of the scattering amplitude. The chiral correction terms of order one renormalize the pole residuum $w$ and add further smooth remainder terms. The pole residuum is:

$$
\begin{aligned}
1-w & =\bar{\kappa}_{J, \pi}^{(1)}\left(z_{0}\right)-2 \bar{\kappa}_{V, \pi}^{(0)}\left(z_{0}\right)+2 \zeta_{2}^{(0)} z_{0} \\
& +\bar{\kappa}_{J, \pi \pi}^{(1)}\left(z_{0}\right)-2 \bar{\kappa}_{V, \pi \pi}^{(0)}\left(z_{0}\right)-\left(\bar{\kappa}_{V, \pi}^{(0)}\left(z_{0}\right)-\bar{\kappa}_{J, \pi}^{(1)}\left(z_{0}\right)\right)^{2} \\
& +4\left(\bar{\kappa}_{V, \pi}^{(0)}\left(z_{0}\right)-\bar{\kappa}_{J, \pi}^{(1)}\left(z_{0}\right)-\zeta_{2}^{(0)} z_{0}\right) \zeta_{2}^{(0)} z_{0} \\
& -2\left(\bar{\kappa}_{V, \pi}^{(1)}\left(z_{0}\right)-\bar{\kappa}_{J, \pi}^{(2)}\left(z_{0}\right)\right) \frac{m}{2 \pi} \zeta_{0}^{(2)} m_{\pi}^{2}+\mathcal{O}\left(Q^{3}\right) .
\end{aligned}
$$

We close this appendix with a more detailed analysis of the low energy behavior of (C.9). A systematic $z_{0} / m_{\pi}$ expansion is worked out. In particular we show results for the low energy coefficients $b_{\text {eff }}^{(0)}$ and $b_{\text {eff }}^{(1)}$ :

$$
\begin{aligned}
b_{\mathrm{eff}}^{(0)} & =-\zeta_{2}^{(0)}-\frac{m}{4 \pi} g_{A}^{2} \lambda^{2}\left(1-4 R_{V, \pi}^{(1)}\left(0, z_{0}\right)+2 R_{J, \pi}^{(2)}\left(0, z_{0}\right)\right) \\
& -\zeta_{2}^{(0)}\left(2 \bar{\kappa}_{V, \pi}^{(0)}\left(z_{0}\right)-2 \bar{\kappa}_{J, \pi}^{(1)}\left(z_{0}\right)-3 \zeta_{2}^{(0)} z_{0}\right)
\end{aligned}
$$




$$
\begin{aligned}
& -g_{A}^{2} \lambda^{2} \zeta_{0}^{(2)} m_{\pi}^{2} \frac{m^{2}}{2 \pi^{2}}\left(R_{J, \pi}^{(3)}\left(0, z_{0}\right)-R_{V, \pi}^{(2)}\left(0, z_{0}\right)\right) \\
& -g_{A}^{2} \lambda^{2}\left(\bar{\kappa}_{V, \pi}^{(0)}\left(z_{0}\right)-\bar{\kappa}_{J, \pi}^{(1)}\left(z_{0}\right)-\zeta_{2}^{(0)} z_{0}\right) \frac{m}{\pi}\left(R_{J, \pi}^{(2)}\left(0, z_{0}\right)-R_{V, \pi}^{(1)}\left(0, z_{0}\right)\right) \\
& -g_{A}^{4} \lambda^{4} \frac{m}{2 \pi}\left(R_{J, \pi \pi}^{(2)}\left(0, z_{0}\right)-2 R_{V, \pi \pi}^{(1)}\left(0, z_{0}\right)\right) \\
& -g_{A}^{4} \lambda^{4} \frac{m^{2} z_{0}}{4 \pi^{2}}\left(R_{V, \pi}^{(1)}\left(0, z_{0}\right)-R_{J, \pi}^{(2)}\left(0, z_{0}\right)\right)^{2} \\
& -g_{A}^{4} \lambda^{4} \frac{m}{2 \pi} R_{\pi \pi}(0)+\mathcal{O}\left(Q^{2}\right) \\
b_{\mathrm{eff}}^{(1)} & =-g_{A}^{2} \lambda^{2} \frac{m}{2 \pi}\left(-i \partial_{p}\right)\left(R_{J, \pi}^{(2)}\left(0, z_{0}\right)-2 R_{V, \pi}^{(1)}\left(0, z_{0}\right)\right)+\left(\zeta_{2}^{(0)}\right)^{2} \\
& -g_{A}^{2} \lambda^{2} \frac{m^{2}}{2 \pi^{2}} \zeta_{0}^{(2)} m_{\pi}^{2}\left(-i \partial_{p}\right)\left(R_{J, \pi}^{(3)}\left(0, z_{0}\right)-R_{V, \pi}^{(2)}\left(0, z_{0}\right)\right) \\
& +g_{A}^{2} \lambda^{2} \frac{m}{\pi} \zeta_{2}^{(0)}\left(R_{J, \pi}^{(2)}\left(0, z_{0}\right)-R_{V, \pi}^{(1)}\left(0, z_{0}\right)\right) \\
& +g_{A}^{2} \lambda^{2} \frac{m}{\pi} \zeta_{2}^{(0)} z_{0}\left(-i \partial_{p}\right)\left(R_{J, \pi}^{(2)}\left(0, z_{0}\right)-R_{V, \pi}^{(1)}\left(0, z_{0}\right)\right) \\
& -g_{A}^{2} \lambda^{2}\left(\bar{\kappa}_{V, \pi}^{(0)}\left(z_{0}\right)-\bar{\kappa}_{J, \pi}^{(1)}\left(z_{0}\right)\right) \frac{m}{\pi}\left(-i \partial_{p}\right)\left(R_{J, \pi}^{(2)}\left(0, z_{0}\right)-R_{V, \pi}^{(1)}\left(0, z_{0}\right)\right) \\
& -g_{A}^{4} \lambda^{4} \frac{m}{2 \pi}\left(-i \partial_{p}\right)\left(R_{J, \pi \pi}^{(2)}\left(0, z_{0}\right)-2 R_{V, \pi \pi}^{(1)}\left(0, z_{0}\right)\right) \\
& -g_{A}^{4} \lambda^{4} \frac{m^{2} z_{0}}{4 \pi^{2}}\left(-i \partial_{p}\right)\left(R_{V, \pi}^{(1)}\left(0, z_{0}\right)-R_{J, \pi}^{(2)}\left(0, z_{0}\right)\right)^{2} \\
& +g_{A}^{4} \lambda^{4} \frac{m^{2}}{4 \pi^{2}}\left(R_{V, \pi}^{(1)}\left(0, z_{0}\right)-R_{J, \pi}^{(2)}\left(0, z_{0}\right)\right)^{2} \\
& -g_{A}^{4} \lambda^{4} \frac{m}{2 \pi}\left(-i \partial_{p}\right) R_{\pi \pi}(0)+\mathcal{O}\left(Q^{2}\right) \\
&
\end{aligned}
$$

introduced when discussing the structure of the scattering amplitude in (27). First the coefficients $\bar{\kappa}_{J, \pi^{k}}^{(l)}(z)$ and $\bar{\kappa}_{V, \pi^{k}}^{(l)}(z)$ are systematically expanded in the small ratio $z / m_{\pi}$ with the leading moments

$$
\begin{aligned}
& \bar{\kappa}_{V, \pi}^{(0)}(z)=g_{A}^{2} \lambda^{2} \frac{m m_{\pi}}{4 \pi}\left(1+\frac{z}{m_{\pi}}+\frac{4}{3} \frac{z^{2}}{m_{\pi}^{2}}+\frac{2 z^{3}}{m_{\pi}^{3}}+\mathcal{O}\left(\frac{z^{4}}{m_{\pi}^{4}}\right)\right), \\
& \bar{\kappa}_{V, \pi}^{(1)}(z)=g_{A}^{2} \lambda^{2}\left(\frac{1}{2}+\frac{4 z}{3 m_{\pi}}+\frac{3 z^{2}}{m_{\pi}^{2}}+\frac{32 z^{3}}{5 m_{\pi}^{3}}+\mathcal{O}\left(\frac{z^{4}}{m_{\pi}^{4}}\right)\right), \\
& \bar{\kappa}_{J, \pi}^{(0)}(z)=g_{A}^{2} \lambda^{2} \frac{m^{2} m_{\pi}^{2}}{(4 \pi)^{2}} \ln \left(\frac{\Lambda^{2}}{m_{\pi}^{2}}\right)\left(1+\mathcal{O}\left(\frac{m_{\pi}}{\Lambda}, \frac{z}{m_{\pi}}\right)\right), \\
& \bar{\kappa}_{J, \pi}^{(1)}(z)=g_{A}^{2} \lambda^{2} \frac{m m_{\pi}}{4 \pi}\left(2+\frac{4 z}{m_{\pi}}+\frac{8 z^{2}}{m_{\pi}^{2}}+\frac{16 z^{3}}{m_{\pi}^{3}}+\mathcal{O}\left(\frac{z^{4}}{m_{\pi}^{4}}\right)\right), \\
& \bar{\kappa}_{J, \pi}^{(2)}(z)=g_{A}^{2} \lambda^{2}\left(1+\frac{4 z}{m_{\pi}}+\frac{12 z^{2}}{m_{\pi}^{2}}+\frac{32 z^{3}}{m_{\pi}^{3}}+\mathcal{O}\left(\frac{z^{4}}{m_{\pi}^{4}}\right)\right)
\end{aligned}
$$




$$
\begin{gathered}
\bar{\kappa}_{V, \pi \pi}^{(0)}(z)=g_{A}^{4} \lambda^{4} \frac{m^{2} m_{\pi}^{2}}{(4 \pi)^{2}}\left(\ln (2)+\frac{3}{2} \frac{z}{m_{\pi}}+\left(\frac{3}{2}+\ln (4)\right) \frac{z^{2}}{m_{\pi}^{2}}+\mathcal{O}\left(\frac{z^{3}}{m_{\pi}^{3}}\right)\right), \\
\bar{\kappa}_{J, \pi \pi}^{(1)}(z)=g_{A}^{4} \lambda^{4} \frac{m^{2} m_{\pi}^{2}}{(4 \pi)^{2}}\left(1+2 \ln (2)+\left(\frac{14}{3}+\frac{16}{3} \ln (2)\right) \frac{z}{m_{\pi}}\right. \\
\left.+(14+12 \ln (2)) \frac{z^{2}}{m_{\pi}^{2}}+\mathcal{O}\left(\frac{z^{3}}{m_{\pi}^{3}}\right)\right) .
\end{gathered}
$$

Next we provide the leading moments of the residual functions

$$
\begin{aligned}
& R_{V, \pi}^{(1)}(p, z)=\left(\frac{1}{2}+\frac{2 z}{3 m_{\pi}}+\frac{z^{2}}{m_{\pi}^{2}}\right) \\
& +i \frac{p}{m_{\pi}}\left(\frac{2}{3}+\frac{z}{m_{\pi}}+\frac{8 z^{2}}{5 m_{\pi}^{2}}\right)+\mathcal{O}\left(\frac{z^{3}}{m_{\pi}^{3}}, \frac{p^{2}}{m_{\pi}^{2}}\right), \\
& R_{V, \pi}^{(2)}(p, z)=\frac{2 \pi}{m m_{\pi}}\left(\frac{2}{3}+\frac{2 z}{m_{\pi}}+\frac{24 z^{2}}{5 m_{\pi}^{2}}\right) \\
& +i \frac{2 \pi}{m m_{\pi}} \frac{p}{m_{\pi}}\left(1+\frac{16 z}{5 m_{\pi}}+\frac{8 z^{2}}{m_{\pi}^{2}}\right)+\mathcal{O}\left(\frac{z^{3}}{m_{\pi}^{3}}, \frac{p^{2}}{m_{\pi}^{2}}\right) \text {, } \\
& R_{J, \pi}^{(2)}(p, z)=\left(1+\frac{8 z}{3 m_{\pi}}+\frac{6 z^{2}}{m_{\pi}^{2}}\right) \\
& +i \frac{p}{m_{\pi}}\left(\frac{4}{3}+\frac{4 z}{m_{\pi}}+\frac{48 z^{2}}{5 m_{\pi}^{2}}\right)+\mathcal{O}\left(\frac{z^{3}}{m_{\pi}^{3}}, \frac{p^{2}}{m_{\pi}^{2}}\right), \\
& R_{J, \pi}^{(3)}(p, z)=\frac{2 \pi}{m m_{\pi}}\left(\frac{4}{3}+\frac{6 z}{m_{\pi}}+\frac{96 z^{2}}{5 m_{\pi}^{2}}\right) \\
& +i \frac{2 \pi}{m m_{\pi}} \frac{p}{m_{\pi}}\left(2+\frac{48 z}{5 m_{\pi}}+\frac{32 z^{2}}{m_{\pi}^{2}}\right)+\mathcal{O}\left(\frac{z^{3}}{m_{\pi}^{3}}, \frac{p^{2}}{m_{\pi}^{2}}\right), \\
& R_{V, \pi \pi}^{(1)}(p, z)=\frac{m m_{\pi}}{4 \pi}\left(\frac{3}{4}+\left(\frac{3}{4}+\ln (2)\right) \frac{z}{m_{\pi}}+\frac{65}{24} \frac{z^{2}}{m_{\pi}^{2}}\right) \\
& +i \frac{m m_{\pi}}{4 \pi}\left(\frac{3}{4}+\ln (2)+\frac{65}{24} \frac{z}{m_{\pi}}+\left(\frac{77}{24}+\frac{8}{3} \ln (2)\right) \frac{z^{2}}{m_{\pi}^{2}}\right) \frac{p}{m_{\pi}} \\
& +\mathcal{O}\left(\frac{z^{3}}{m_{\pi}^{3}}, \frac{p^{2}}{m_{\pi}^{2}}\right) \\
& R_{J, \pi \pi}^{(2)}(p, z)=\frac{m m_{\pi}}{4 \pi}\left(\frac{7}{6}+\frac{4}{3} \ln (2)+\left(\frac{14}{3}+4 \ln (2)\right) \frac{z}{m_{\pi}}\right. \\
& \left.+\left(\frac{269}{20}+\frac{48}{5} \ln (2)\right) \frac{z^{2}}{m_{\pi}^{2}}\right) \\
& +i \frac{m m_{\pi}}{4 \pi}\left(\frac{7}{3}+2 \ln (2)+\left(\frac{269}{30}+\frac{32}{5} \ln (2)\right) \frac{z}{m_{\pi}}\right. \\
& \left.+\left(\frac{1531}{60}+16 \ln (2)\right) \frac{z^{2}}{m_{\pi}^{2}}\right) \frac{p}{m_{\pi}}+\mathcal{O}\left(\frac{z^{3}}{m_{\pi}^{3}}, \frac{p^{2}}{m_{\pi}^{2}}\right)
\end{aligned}
$$


Finally note the low energy characteristics of the s-wave projected box function

$$
R_{\pi \pi}(p)=\frac{m m_{\pi}}{4 \pi}\left(\frac{1}{4}+\frac{i p}{2 m_{\pi}}+\mathcal{O}\left(\frac{p^{2}}{m_{\pi}^{2}}\right)\right)
$$

Applying the our results (C.12) and (C.13) we derive

$$
\begin{aligned}
& 1-w=\frac{m m_{\pi}}{4 \pi} g_{A}^{2} \lambda^{2}\left(2 \frac{z_{0}}{m_{\pi}}+\frac{16}{3} \frac{z_{0}^{2}}{m_{\pi}^{2}}+\mathcal{O}\left(\frac{z_{0}^{3}}{m_{\pi}^{3}}\right)\right)+2 \zeta_{2}^{(0)} z_{0} \\
& +\left(g_{A}^{2} \lambda^{2} \frac{m m_{\pi}}{4 \pi}\right)^{2}\left(\left(-\frac{13}{3}+\frac{16}{3} \ln (2)\right) \frac{z_{0}}{m_{\pi}}\right. \\
& \left.+\left(-\frac{34}{3}+8 \ln (2)\right) \frac{z_{0}^{2}}{m_{\pi}^{2}}+\mathcal{O}\left(\frac{z_{0}^{3}}{m_{\pi}^{3}}\right)\right) \\
& -4 g_{A}^{2} \lambda^{2} \frac{m m_{\pi}}{4 \pi}\left(1+\frac{3 z_{0}}{m_{\pi}}+\mathcal{O}\left(\frac{z_{0}^{2}}{m_{\pi}^{2}}\right)\right) \zeta_{2}^{(0)} z_{0} \\
& +2 g_{A}^{2} \lambda^{2} \frac{m m_{\pi}}{4 \pi}\left(1+\frac{16 z_{0}}{3 m_{\pi}}+\frac{18 z_{0}^{2}}{m_{\pi}^{2}}+\mathcal{O}\left(\frac{z_{0}^{3}}{m_{\pi}^{3}}\right)\right) \zeta_{0}^{(2)} m_{\pi} \\
& -4 \zeta_{2}^{(0)} z_{0} \zeta_{2}^{(0)} z_{0}+\mathcal{O}\left(Q^{3}\right) \\
& b_{\mathrm{eff}}^{(0)}=-\zeta_{2}^{(0)}-\frac{m}{4 \pi} g_{A}^{2} \lambda^{2}\left(1+\frac{8}{3} \frac{z_{0}}{m_{\pi}}+\frac{8 z_{0}^{2}}{m_{\pi}^{2}}+\mathcal{O}\left(\frac{z_{0}^{3}}{m_{\pi}^{3}}\right)\right) \\
& +g_{A}^{4} \lambda^{4} \frac{m^{2} m_{\pi}}{(4 \pi)^{2}}\left(\frac{13}{6}-\frac{8}{3} \ln (2)+\left(\frac{20}{3}-4 \ln (2)\right) \frac{z_{0}}{m_{\pi}}\right. \\
& \left.+\left(\frac{499}{15}-\frac{96}{5} \ln (2)\right) \frac{z_{0}^{2}}{m_{\pi}^{2}}+\mathcal{O}\left(\frac{z_{0}^{3}}{m_{\pi}^{3}}\right)\right) \\
& -g_{A}^{2} \lambda^{2} \frac{m m_{\pi}}{4 \pi} \zeta_{0}^{(2)}\left(\frac{8}{3}+\frac{16 z_{0}}{m_{\pi}}+\frac{288 z_{0}^{2}}{5 m_{\pi}^{2}}+\mathcal{O}\left(\frac{z_{0}^{3}}{m_{\pi}^{3}}\right)\right) \\
& +g_{A}^{2} \lambda^{2} \frac{m m_{\pi}}{4 \pi} \zeta_{2}^{(0)}\left(2+\frac{8 z_{0}}{m_{\pi}}+\frac{64 z_{0}^{2}}{3 m_{\pi}^{2}}+\mathcal{O}\left(\frac{z_{0}^{3}}{m_{\pi}^{3}}\right)\right) \\
& +3 z_{0}\left(\zeta_{2}^{(0)}\right)^{2}+\mathcal{O}\left(Q^{2}\right) \\
& b_{\mathrm{eff}}^{(1)}=-\frac{z_{0} m}{\pi m_{\pi}^{2}} g_{A}^{2} \lambda^{2}\left(1+\frac{16}{5} \frac{z_{0}}{m_{\pi}}+\mathcal{O}\left(\frac{z_{0}^{2}}{m_{\pi}^{2}}\right)\right) \\
& +g_{A}^{4} \lambda^{4} \frac{m^{2}}{(4 \pi)^{2}}\left(1+\left(\frac{547}{30}-\frac{64}{5} \ln (2)\right) \frac{z_{0}}{m_{\pi}}\right. \\
& \left.+\left(\frac{2741}{45}-\frac{64}{3} \ln (2)\right) \frac{z_{0}^{2}}{m_{\pi}^{2}}+\mathcal{O}\left(\frac{z_{0}^{3}}{m_{\pi}^{3}}\right)\right) \\
& -g_{A}^{2} \lambda^{2} \frac{m}{4 \pi} \zeta_{0}^{(2)}\left(4+\frac{128 z_{0}}{5 m_{\pi}}+\frac{96 z_{0}^{2}}{m_{\pi}^{2}}+\mathcal{O}\left(\frac{z_{0}^{3}}{m_{\pi}^{3}}\right)\right)
\end{aligned}
$$




$$
\begin{aligned}
& +g_{A}^{2} \lambda^{2} \frac{m}{4 \pi} \zeta_{2}^{(0)}\left(2+\frac{32 z_{0}}{3 m_{\pi}}+\frac{32 z_{0}^{2}}{m_{\pi}^{2}}+\mathcal{O}\left(\frac{z_{0}^{3}}{m_{\pi}^{3}}\right)\right) \\
& +\left(\zeta_{2}^{(0)}\right)^{2}+\mathcal{O}\left(Q^{2}\right) .
\end{aligned}
$$

\section{References}

[1] M. Lutz in, proceedings of the workshop "The standard Model at Low Energy" held at ECT*, April 29 to May 10, 1996, hep-ph/9606301; M. Lutz, Nucl. Phys. A 642 (1998) 171c.

[2] K.A. Brückner and K.M. Watson, Phys. Rev. 92 (1953) 1023.

[3] R. Machleidt, Adv. Nucl. Phys. 19 (1989) 189.

[4] V.G.J. Stoks, R.A.M. Klomp, C.P.F. Terheggen and J.J. de Swart, Phys. Rev. C 49 (1994) 2950.

[5] M. Lacombe et al., Phys. Rev. C 21 (1980) 861.

[6] D. Plümper, J. Flender and M. F. Gari, Phys. Rev. C 49 (1994) 2370.

[7] S. Weinberg, Phys. Lett. B 251(1990) 288; Nucl. Phys. B 363 (1991) 3.

[8] C. Ordonez and U. van Kolck, Phys. Lett. B 291 (1992) 459.

[9] C. Ordonez, L. Ray and U. van Kolck, Phys. Rev. Lett. 13 (1994) 1982; Phys. Rev. C 53 (1996) 2086.

[10] T.-S. Park, K. Kubodera, D.-P. Min and M. Rho, Nucl. Phys. A 646 (1999) 83.

[11] E. Epelbaoum, W. Glöckle, A. Krüger and U.-G. Meißner, Nucl. Phys. A 645 (1999) 413.

[12] U. van Kolck, Prog. Part. Nucl. Phys. 43 (1999) 337.

[13] J. Gegelia, nucl-th/9806028v2.

[14] J.V. Steele and R.J. Furnstahl, Nucl. Phys. A 645 (1999) 439.

[15] D.B. Kaplan, M.J. Savage and M.B. Wise, Nucl. Phys. B 534 (1998) 329; T. Mehen and I. Stewart, nucl-th/9809095; M.C. Birse, J.A. McGovern and K.G. Richardson, nucl-th/9808398.

[16] T.D. Cohen and J. M. Hansen, nucl-th/9808038V3.

[17] J. Gasser, M.E. Sainio and A. Svarc, Nucl. Phys. B 307 (1988) 779.

[18] A. Krause, Helv. Phys. Acta 63 (1990) 3.

[19] E. Jenkins and A. Manohar, Phys. Lett. B 255 (1991) 558. 
[20] V. Bernard, N. Kaiser and U.-G. Meißner, Int. Jour. of Mod. Phys. E 4 (1995) 193; T.-S. Park, D.-P. Min and M. Rho, Phys. Rep. 233 (1993) 341, N Fettes, U.-G. Meißner and S. Steininger, Nucl.Phys. A 640 (1998) 199.

[21] S. Weinberg, Phys. Rev. 1661568 (1968); Physica 96 A (1979) 327.

[22] G.P. Lepage, nucl-th/9706029.

[23] R.E. Cutkosky, J. Math. Phys. 1 (1960) 429.

[24] G.E. Brown and A.D. Jackson, The Nucleon-Nucleon Interaction (NorthHolland, Amsterdam, 1976).

[25] R. V. Reid, Ann. Phys. 50 (1968) 411.

[26] U. van Kolck, Nucl.Phys. A 645 (1999) 273.

[27] J.M. Charap, Phys. Rev. D 2 (1970) 1554; D 3 (1971) 1998; J. Honerkamp and K. Meetz, D 3 (1971) 1996. I. Gerstein, R. Jackiw, B.W. Lee and S. Weinberg, D 3 (1971) 2486;

[28] S.R. Beane, D.R. Phillips and T.D. Cohen, Nucl. Phys. A 632 (1998) 445.

[29] K-G. Fogel, Arkiv f. Fysik 42 (1952) 4.

[30] A. Friman, Acta Acad. Aboensis Math. et Phys. 41 (1957) 21.

[31] T. Mehen and I.W. Stewart, nucl-th/9906010; G. Rupak and N. Shoresh, nuclth/9902077.

[32] V.G.J. Stoks et al., Phys. Rev. C 48 (1993) 792; http://nn-online.sci.kun.nl.

[33] R.A. Arndt et al., Phys. Rev. C 56 (1997) 3005; http://said.phys.vt.edu.

[34] N. Kaiser, R. Brockmann, W. Weise, Nucl.Phys. A 625 (1997) 758.

[35] M. Jacob and G.C. Wick, Ann. Phys. 7 (1959) 404.

[36] M. Mojzis, Eur. Phys. J C 2 (1998) 181.

[37] P.J. Ellis and H-B. Tang, Phys. Rev. C 57 (1998) 3356.

[38] T. Becher and H. Leutwyler, Eur. Phys. J. C 9 (1999) 643.

[39] T.D. Cohen et al., Phys. Rev. C 53 (1996) 2661.

[40] U. van Kolck, G.A. Miller and D.O. Riska, Phys. Lett. B 388 (1996) 679.

[41] B.-Y. Park et al., Phys. Rev. C 53 (1996) 1519.

[42] C. Hanart et al., Phys. Lett. B 424 (1998) 8.

[43] C.A. da Rocha, G.A. Miller and U. van Kolck, nucl-th/9904031.

[44] V. Dimitrasinovic et al., nucl-th/9902048.

[45] A.M. Green, J.A. Niskanen and M.E. Sainio, J. Phys. G 4 (1978) 1055. 
[46] T.-S. Lee and D.O. Riska, Phys. Rev. Lett. 70 (1993) 2237.

[47] C.J. Horowitz, H.O. Meyer and D.K. Griegel, Phys. Rev. C 49 (1994) 1337.

[48] B. Blankleider, PSI-PR-91-01; A.M. Green in, Mesons in Nuclei, Eds. M. Rho and D.H. Wilkinson (1979).

[49] H.P. Stapp and T.J. Ypsilantis, Phys. Rev. 105 (1957) 302.

[50] J. Gegelia, G. Japaridze and X.Q. Wang, hep-ph/9910260. 